\title{
An efficient and reliable residual-type a posteriori error estimator for the Signorini problem
}

\author{
Rolf Krause · Andreas Veeser · Mirjam Walloth
}

Received: date / Accepted: date

\begin{abstract}
We derive a new a posteriori error estimator for the Signorini problem. It generalizes the standard residual-type estimators for unconstrained problems in linear elasticity by additional terms at the contact boundary addressing the non-linearity. Remarkably these additional contact-related terms vanish in the case of so-called full-contact. We prove reliability and efficiency for two- and three-dimensional simplicial meshes. Moreover, we address the case of non-discrete gap functions. Numerical tests for different obstacles and starting grids illustrate the good performance of the a posteriori error estimator in the twoand three-dimensional case, for simplicial as well as for unstructured mixed meshes.
\end{abstract}

Keywords Signorini problem · residual-type a posteriori error estimator · Galerkin functional $\cdot$ full-contact $\cdot$ adaptive finite elements

\section{Introduction}

Mesh adaption is an important tool in the numerical simulation of real world problems as, e.g., arising from mechanics or biomechanics. In fact it often dramatically improves the quality of the numerical solution for given computational resources. It is usually implemented by an iterative process that, with the help of a so-called a posteriori error estimator, determines regions with less regular or even singular behaviour of the unknown exact solution. For this purpose, the estimator should be equivalent to the error, at least up to so-called oscillation terms of higher order. In the case of linear elliptic boundary value problems from

Rolf Krause

Institute of Computational Science, University of Lugano

Via Giuseppe Buffi 13, CH-6900 Lugano

E-mail: rolf.krause@usi.ch

Andreas Veeser

Dipartimento di Matematica, Università degli Studi di Milano

Via C. Saldini 50, I-20133 Milano

E-mail: andreas.veeser@unimi.it

Mirjam Walloth

Fachbereich Mathematik, Technische Universität Darmstadt

Dolivostraße 15, D-64293 Darmstadt

E-mail: walloth@mathematik.tu-darmstadt.de 
mechanics, the construction of such a posteriori error estimators has reached a certain maturity - we refer to, e.g., [1,33,34], for an overview. Roughly speaking, the construction consists of the following two steps: the establishment of an equivalence of the error and an appropriate dual norm of the residual and the estimation from above and below of that dual norm. For the second step, various techniques are available. A popular one, which appears attractive in view of its simplicity and generality, is standard residual estimation.

We are interested in Signorini problems from contact mechanics which are characterized by inequality constraints arising from a non-penetration condition. There are two closely related problems: the simplified Signorini problem, where the unknown is scalar-valued instead of vector-valued, and the obstacle problem, where the inequality constraints on the scalar-valued unknown are not imposed on a part of the boundary but on the whole domain. Some of the estimation techniques for linear elliptic problems have been extended to obstacle problems, see $[3,5,7,9,16,20,22,24-27,29,31,37,39]$, and (simplified) Signorini problems, see $[14,15,36,38]$. The works on the obstacle problem reveal that sharp a posteriori error estimators for such nonlinear problems have to invoke some approximation of the exact Lagrange multiplier. From the physical point of view, the latter corresponds to a constraining or contact force. In this manner the estimator uses an approximation of the actual contact region, taking into account its possible instability. Regarding the works on the (simplified) Signorini problem, notice that all mentioned works are limited to two-dimensional reference configurations and that in [14], which considers the same discretization and estimator types as in this article, the given upper and lower bounds do not ensure equivalence of error and estimator.

In this article we propose, analyze and test a new a posteriori error estimator for the linear finite element solution of the Signorini problem in linear elasticity. The discrete problem consists in a discrete variational inequality for the approximate displacement (Section 2) and the estimator is designed for controlling its $H^{1}$-error plus the $H^{-1}$-error of a suitable approximation of the Lagrange multiplier (Section 3). The estimator reduces to the standard residual estimator for linear elasticity, if no contact occurs. The estimator contributions addressing the nonlinearity are related to the contact stresses, the complementarity condition, and the approximation of the gap function. Remarkably, the first two terms vanish also if the actual discrete contact is 'full' in that the sign of the contact stresses is compatible with actual contact of the exact solution. We prove reliability (Section 4) and efficiency (Section 5) of the estimator for simplicial meshes, ensuring the equivalence with the error up to oscillation terms. We thus reproduce the estimator properties encountered for obstacle problems. The theoretical results are corroborated by a variety of numerical tests, addressing also non-simplicial meshes (Section 6). Analysis and numerical tests cover two-dimensional and three-dimensional reference configurations, as well as arbitrary, non-discrete gap functions.

In order to derive and analyze the error estimator, we adopt the framework presented in [31] and used in, e.g., [12,24-26] for obstacle problems. A key ingredient of this approach is the so-called Galerkin functional. It is a modification of the residual with respect to the corresponding linear problem with the help of a suitable approximation of the Lagrange multiplier and, thus, may be seen as the residual of a linear auxiliary problem [6]. The correction by the Lagrange multiplier is crucial for sharpness of the upper bounds in the actual contact regions.

The following comments compare and highlight the differences of our implementation of this approach for the Signorini problem to the preceding ones for obstacle problems.

- The sharpness of the resulting upper bounds with respect to the displacement error hinges on the approximate Lagrange multiplier. In $[12,24,26]$ the approximate Lagrange 
multiplier is constructed from the discrete solution and given data with help of the partition of unity given by the canonical basis function. Notice that, generically, the support of the Lagrange multiplier is $d$-dimensional for obstacle problems, while it is $(d-1)$ dimensional for Signorini problems. Since the basis functions have $d$-dimensional support, this entails that the identification of the correct contributions for the approximate Lagrange multiplier is complicated by averaging on the discrete level for the Signorini problem. Our construction in Section 3.1 deals with this difficulty by invoking appropriate means of the test function, which are chosen in a nonlinear manner in the case of full-contact. The construction is confirmed by the following three facts from Section 4. The derivation of the upper bound for the dual norm of the Galerkin functional goes along the usual lines. The resulting estimator contributions addressing the inequality constraints vanish on full-contact regions. The consistency error of the approximate Lagrange multiplier is bounded in terms related to the complementarity condition.

- Generally speaking, the derivation of a lower bound in terms of the estimator contribution related to contact stresses follows Verfürth's constructive argument [33] by suitably testing the Galerkin functional. The aforementioned means in the definition of the approximate Lagrange multiplier complicate matters. We obtain the appropriate test functions in Subsection 5.1 as special linear combinations of bubble functions over virtual subgrids.

- For obstacle problems, the lower bound in terms of the estimator contribution associated with the complementarity condition is established by a discrete counterpart of the critical point argument by Baiocchi [2]. It allows to bound the detachment of the discrete solution from the obstacle by the jumps in the normal derivatives across inter-element faces. In Subsection 5.2 we provide the counterpart of this for linear elasticity. More precisely, we bound the discrete detachment from the obstacle by jumps across interelement faces of the discrete stress in normal direction and by corresponding jumps of a suitable extension of the gap function.

Our theoretical findings are supported by intensive numerical studies. The adaptively refined grids and the relevance of the different error estimator contributions are analyzed by means of different illustrative numerical experiments in $3 \mathrm{D}$. We also quantitatively investigate the convergence of the error estimator by comparing to the case of uniformly refined grids. Furthermore, for selected examples in $2 D$ and even in $3 D$ where the contact stresses are known analytically, we compare the numerically computed contact stresses on adaptively refined grids with the exact contact stresses. Interestingly, although the proofs of upper and lower bound are given for simplicial meshes, the numerical studies show also very good performance of the new residual-type a posteriori error estimator for unstructured mixed meshes consisting of triangles and quadrilaterals in $2 D$ and hexahedra, tetrahedra, prisms, and pyramids in $3 D$.

\section{The Signorini contact problem}

The Signorini contact problem describes the contact of a linear elastic body with a rigid obstacle. The linear elastic body is represented by a domain $\Omega \subset \mathbb{R}^{d}, d=2,3$. The boundary $\Gamma=\partial \Omega$ is subdivided in three pairwise disjoint parts, the Neumann boundary $\Gamma_{N}$ which is an open subset of $\Gamma$, the Dirichlet boundary $\Gamma_{D}$ which is a closed subset of $\Gamma$ and the potential contact boundary $\Gamma_{C}$ which is also a closed subset. Each material particle in the closure $\bar{\Omega}$ is identified with a point $\boldsymbol{x}=\left(x_{1}, \ldots, x_{d}\right)^{T}$. Throughout this work we denote all 
quantities which refer to tensors of order $\geq 1$ by bold symbols as, e.g., the displacements $\boldsymbol{u}$ which are vector-valued. Their components are printed in normal type and are indicated by subindices, e.g., $u_{i}$. The summation convention is enforced and $e_{i}, i=1, \ldots, d$, denote the Cartesian basis vectors of $\mathbb{R}^{d}$ such that, e.g., $\boldsymbol{u}=u_{i} \boldsymbol{e}_{i}$.

In linear elasticity [10] small displacements and small strains allow for linearization of the strain-displacement relation as well as of the stress-strain relation of elastic materials. The stress $\boldsymbol{\sigma}(\boldsymbol{u}): \Omega \rightarrow \mathbb{R}^{d \times d}$ evoked by the displacements $\boldsymbol{u}: \Omega \rightarrow \mathbb{R}^{d}$ obeys Hooke's law

$$
\sigma_{i j}(\boldsymbol{u})=E_{i j m l} \epsilon_{m l}(\boldsymbol{u})
$$

where the strain tensor is given by

$$
\boldsymbol{\epsilon}(\boldsymbol{u})=\frac{1}{2}\left(\nabla \boldsymbol{u}+(\nabla \boldsymbol{u})^{T}\right)
$$

and $E_{i j m l}$ are the components of Hooke's tensor which is symmetric, elliptic and bounded. We note that (1) can be expressed by means of the Lamé constants $\lambda$ and $\mu$ as follows:

$$
\boldsymbol{\sigma}=\lambda \operatorname{tr}(\boldsymbol{\epsilon}) \boldsymbol{i d}+2 \mu \boldsymbol{\epsilon} .
$$

When two solid bodies come into contact they do not penetrate each other. As the actual contact zone depends on the deformation of the elastic body, which is an unknown of the system we do not know in advance which points are coming into contact. However, if the displacements are small like in linear elasticity we can approximate the distance a priori [11], e.g. by means of a closest point projection, cf., [19]. The gap function describing the distance between the elastic body and the rigid body is given by $g: \Gamma_{C} \rightarrow \mathbb{R}$ and the direction of constraints are denoted by $\nu$. Thus, the non-penetration condition is

$$
u_{\nu} \leq g \text { on } \Gamma_{C}
$$

where $u_{\nu}:=\boldsymbol{u} \cdot \boldsymbol{\nu}$. The non-penetration condition evokes so-called contact stresses which are boundary stresses in direction of the constraints at the actual contact boundary. We use the notation $\hat{\boldsymbol{\sigma}}(\boldsymbol{u}):=\boldsymbol{\sigma}(\boldsymbol{u}) \boldsymbol{n}$ for boundary stresses where $\boldsymbol{n}$ is the unit outward normal to the boundary. Hence, the contact stresses are given by $\hat{\sigma}_{\nu}(\boldsymbol{u}):=\hat{\boldsymbol{\sigma}}(\boldsymbol{u}) \cdot \boldsymbol{\nu}$. As we neglect frictional effects the frictional stresses $\hat{\sigma}_{T}(\boldsymbol{u}):=\hat{\boldsymbol{\sigma}}(\boldsymbol{u})-\hat{\sigma}_{\nu}(\boldsymbol{u}) \cdot \boldsymbol{\nu}$ are assumed to be zero.

In order to enforce a deformation of the linear elastic body, Dirichlet conditions $\boldsymbol{u}=\boldsymbol{u}_{D}$ on $\Gamma_{D}$, Neumann conditions $\hat{\boldsymbol{\sigma}}(\boldsymbol{u})=\boldsymbol{\pi}$ on $\Gamma_{N}$ and a force density $\boldsymbol{f}$ can be applied on $\Omega$. The complete problem formulation is given in Problem 1.

Problem 1 Strong formulation of the Signorini contact problem Find $\boldsymbol{u}: \bar{\Omega} \rightarrow \mathbb{R}^{d}$ such that

$$
\begin{aligned}
-\operatorname{div} \boldsymbol{\sigma}(\boldsymbol{u}) & =\boldsymbol{f} & \text { in } \Omega \\
\hat{\boldsymbol{\sigma}}(\boldsymbol{u}) & =\boldsymbol{\pi} & \text { on } \Gamma_{N} \\
\boldsymbol{u} & =\boldsymbol{u}_{D} & \text { on } \Gamma_{D} \\
u_{\nu} & \leq g & \text { on } \Gamma_{C} \\
\hat{\sigma}_{\nu}(\boldsymbol{u}) & \leq 0 & \text { on } \Gamma_{C} \\
\left(u_{\nu}-g\right) \cdot \hat{\sigma}_{\nu}(\boldsymbol{u}) & =0 & \text { on } \Gamma_{C} \\
\hat{\boldsymbol{\sigma}}_{T}(\boldsymbol{u}) & =0 & \text { on } \Gamma_{C} .
\end{aligned}
$$

As the boundary segments are disjoint, $\Gamma_{D} \cap \Gamma_{C}=\emptyset$. Further, we assume that the actual contact boundary, where $u_{\nu}=g$, is a strict subset of the potential contact boundary. 


\subsection{Weak formulation}

The solution space of the weak formulation is the subspace

$$
\mathcal{H}:=\left\{\boldsymbol{v} \in \boldsymbol{H}^{1}(\Omega)|\operatorname{tr}|_{\Gamma_{D}}(\boldsymbol{v})=\boldsymbol{u}_{D}\right\}
$$

of $\boldsymbol{H}^{1}(\Omega):=\left(H^{1}(\Omega)\right)^{d}$ where tr is the trace operator. For convenience in discretizing $\boldsymbol{u}_{D}$, we assume $\boldsymbol{u}_{D} \in \mathrm{C}^{0}\left(\Gamma_{D}\right)$. Whenever it is clear from the context that the restriction to the boundary requires the trace operator we omit the special notation. The space of test functions is given by

$$
\mathcal{H}_{0}:=\left\{\varphi \in \boldsymbol{H}^{1}(\Omega)|\operatorname{tr}|_{\Gamma_{D}}(\varphi)=0\right\}
$$

and its dual is $\mathcal{H}^{*}$. For a gap function $g \in H^{\frac{1}{2}}\left(\Gamma_{C}\right)$ we define the admissible set

$$
\mathcal{K}:=\left\{\boldsymbol{v} \in \mathcal{H} \mid v_{\nu} \leq g \text { on } \Gamma_{C}\right\}
$$

which is non-empty as $\Gamma_{D} \cap \Gamma_{C}=\emptyset$. The order relation $\leq$ in (3) has to be understood in the sense of almost everywhere with respect to the $(d-1)$-dimensional Hausdorff measure.

We assume the force density $f$ and the Neumann data $\pi$ to be $L^{2}$-functions on $\Omega$ or $\Gamma_{N}$, respectively. Further, the directions of constraints $\nu$ are given by a measurable vector field with absolute value $|\boldsymbol{\nu}(\boldsymbol{x})|=1$. The $L^{2}$-norm and its scalar product are denoted by $\|\cdot\|$ and $\langle\cdot, \cdot\rangle$ without any subindex. The duality pairing between $H^{1}$ and its dual $H^{-1}$ is given by $\langle\cdot, \cdot\rangle_{-1,1}$ and the corresponding norms are $\|\cdot\|_{1}$ and $\|\cdot\|_{-1}$. The duality pairing between $H^{\frac{1}{2}}$ and its dual $H^{-\frac{1}{2}}$ is denoted with $\langle\cdot, \cdot\rangle_{-\frac{1}{2}, \frac{1}{2}}$ and the corresponding norms are $\|\cdot\|_{\frac{1}{2}}$ and $\|\cdot\|_{-\frac{1}{2}}$. Later on, we need restrictions to subdomains which are indicated by a further subindex, e.g., $\|\cdot\|_{1, \omega}$ for $\omega \subset \Omega$. Finally, we define the symmetric bilinear form

$$
a(\cdot, \cdot):=\int_{\Omega} \boldsymbol{\sigma}(\cdot): \epsilon(\cdot),
$$

which is associated with the elastic energy.

The variational inequality in Problem 2 may be derived from the strong formulation (Problem 1) by integration by parts and exploiting $\hat{\sigma}_{\nu}(\boldsymbol{u})\left(v_{\nu}-u_{\nu}\right) \geq 0$ for $\boldsymbol{u}, \boldsymbol{v} \in \mathcal{K}$.

Problem 2 Variational inequality of the Signorini problem

We seek a solution $\boldsymbol{u} \in \mathcal{K}$ such that

$$
a(\boldsymbol{u}, \boldsymbol{v}-\boldsymbol{u}) \geq\langle\boldsymbol{f}, \boldsymbol{v}-\boldsymbol{u}\rangle+\langle\boldsymbol{\pi}, \boldsymbol{v}-\boldsymbol{u}\rangle_{\Gamma_{N}} \quad \forall \boldsymbol{v} \in \mathcal{K}
$$

The unique solvability of Problem 2 follows from the Theorem of Lions and Stampacchia, see e.g., [19, Theorem 2.1].

It exists a distribution $\lambda \in \mathcal{H}^{*}$ which turns the variational inequality (5) in an equation

$$
\langle\boldsymbol{f}, \boldsymbol{\varphi}\rangle+\langle\boldsymbol{\pi}, \boldsymbol{\varphi}\rangle_{\Gamma_{N}}-a(\boldsymbol{u}, \boldsymbol{\varphi})=\langle\boldsymbol{\lambda}, \boldsymbol{\varphi}\rangle_{-1,1} \quad \forall \boldsymbol{\varphi} \in \mathcal{H}_{0} .
$$

From the optimization point of view $\lambda$ is the Lagrange multiplier while from the physical point of view $\lambda$ has the meaning of a constraining force density on $\Gamma_{C}$ which we call contact force density. The contact force density is directly related to the contact stresses

$$
\langle\boldsymbol{\lambda}, \boldsymbol{\varphi}\rangle_{-1,1}=-\left\langle\hat{\sigma}_{\nu}(\boldsymbol{u}), \varphi_{\nu}\right\rangle_{-\frac{1}{2}, \frac{1}{2}}
$$

which follows from the generalized Green's formula, see e.g., [19, Theorem 5.9]. Due to the variational inequality the contact force density fulfills the weak sign condition $\langle\boldsymbol{\lambda}, \boldsymbol{v}-\boldsymbol{u}\rangle_{-1,1} \leq$ 0 . We note that Problem 2 can be equivalently formulated as saddle point of a Lagrangian. The Lagrange multiplier of this saddle point formulation is a functional on $H^{\frac{1}{2}}\left(\Gamma_{C}\right)$ and, hence, formally different to the contact force density. 


\subsection{Discrete formulation}

In the discrete setting we assume the domain $\Omega$ to be polygonal. The mesh $\mathfrak{m}$, resolving the domain, consists of elements $\mathfrak{e} \in \mathfrak{m}$ which are either disjoint or share a node $\boldsymbol{p}$, an edge or a face $\mathfrak{s}$. The polygonal boundary segments $\Gamma_{D}, \Gamma_{C}, \Gamma_{N}$ are resolved by the mesh, too, meaning that their boundaries $\partial \Gamma_{C}, \partial \Gamma_{N}, \partial \Gamma_{D}$ are either nodes or edges. The set of nodes $\boldsymbol{p}$ is given by $N_{\mathfrak{m}}$ and we distinguish between the set $N_{\mathfrak{m}}^{D}$ of nodes on the Dirichlet boundary, the set $N_{\mathfrak{m}}^{N}$ of nodes at the Neumann boundary, the set $N_{\mathfrak{m}}^{C}$ of nodes at the potential contact boundary and the set of interior nodes $N_{\mathfrak{m}}^{I}$ where $\boldsymbol{p} \in \Omega$. The mesh is taken from a shape-regular family, meaning that the ratio of the diameter of any element to the diameter of its inscribed circle is uniformly bounded. We recall that our theoretical results are proven for unstructured simplicial meshes whereas the numerical studies are performed for unstructered mixed meshes consisting of triangles and quadrilaterals in $2 D$ and tetrahedra, hexahedra, prisms and pyramids in $3 D$.

For the approximation of $\mathcal{H}$, we use linear finite elements. The space of linear finite elements which are zero on the Dirichlet boundary is denoted with

$$
\mathcal{H}_{\mathfrak{m}, 0}:=\left\{\boldsymbol{\varphi}_{\mathfrak{m}} \in \mathfrak{C}^{0}(\bar{\Omega})\left|\forall \mathfrak{e} \in \mathfrak{m}, \varphi_{\mathfrak{m}}\right|_{\mathfrak{e}} \in \boldsymbol{P}_{1}(\mathfrak{e}) \text { and } \varphi_{\mathfrak{m}}=0 \text { on } \Gamma_{D}\right\}
$$

and the space with incorporated Dirichlet values $\boldsymbol{u}_{D}$ is

$$
\mathcal{H}_{\mathfrak{m}}:=\left\{\boldsymbol{v}_{\mathfrak{m}} \in \mathfrak{C}^{0}(\bar{\Omega})\left|\forall \mathfrak{e} \in \mathfrak{m}, \boldsymbol{v}_{\mathfrak{m}}\right|_{\mathfrak{e}} \in \boldsymbol{P}_{1}(\mathfrak{e}) \text { and } \boldsymbol{v}_{\mathfrak{m}}=\boldsymbol{u}_{D} \text { on } \Gamma_{D}\right\}
$$

where we assumed $\boldsymbol{u}_{D}$ to be a linear finite element function. The nodal basis functions of the finite element spaces are denoted by $\phi_{p}$. Hence, a discrete vector quantity has the representation

$$
\boldsymbol{\varphi}_{\mathfrak{m}}=\sum_{p \in N_{\mathfrak{m}}} \sum_{i=1}^{d} \varphi_{\mathfrak{m}, i}(\boldsymbol{p}) \phi_{p} \boldsymbol{e}_{i} .
$$

We assume the direction of constraints $\boldsymbol{\nu}$ to be constant so that $v_{\mathfrak{m}, \nu}=\boldsymbol{v}_{\mathfrak{m}} \cdot \boldsymbol{\nu}$ is a linear finite element function on $\Gamma_{C}$. Let $g_{\mathfrak{m}}$ be a discrete approximation of the gap function $g$, then the discrete admissible set is given by

$$
\mathcal{K}_{\mathfrak{m}}:=\left\{\boldsymbol{v}_{\mathfrak{m}} \in \mathcal{H}_{\mathfrak{m}} \mid v_{\mathfrak{m}, \nu} \leq g_{\mathfrak{m}} \text { on } \Gamma_{C}\right\} .
$$

Under these assumptions $\mathcal{K}_{\mathfrak{m}} \subset \mathcal{K}$ if $g=g_{\mathfrak{m}}$.

Problem 3 Discrete variational inequality of the Signorini problem Find $\boldsymbol{u}_{\mathfrak{m}} \in \mathcal{K}_{\mathfrak{m}}$ fulfilling the variational inequality

$$
a\left(\boldsymbol{u}_{\mathfrak{m}}, \boldsymbol{v}_{\mathfrak{m}}-\boldsymbol{u}_{\mathfrak{m}}\right) \geq\left\langle\boldsymbol{f}, \boldsymbol{v}_{\mathfrak{m}}-\boldsymbol{u}_{\mathfrak{m}}\right\rangle+\left\langle\boldsymbol{\pi}, \boldsymbol{v}_{\mathfrak{m}}-\boldsymbol{u}_{\mathfrak{m}}\right\rangle_{\Gamma_{N}} \quad \forall \boldsymbol{v}_{\mathfrak{m}} \in \mathcal{K}_{\mathfrak{m}} .
$$

The unique solvability of the discrete Signorini problem follows just as in the continuous case from the Theorem of Lions and Stampacchia. A priori error estimates have been proven in, e.g., $[8,17]$ for a sufficiently regular continuous solution $\boldsymbol{u}$.

\section{Main Results}

In this section we state the upper and lower a posteriori error estimates but postpone the proofs to Sections 4 and 5. To this end we introduce the error measure and the Galerkin functional and we define the error estimator contributions. The Galerkin functional is defined by means of a quasi-discrete contact force density which mimics the properties of the continuous contact force density $\boldsymbol{\lambda}$ while computed from the discrete solution and given data. 
3.1 Galerkin functional and quasi-discrete contact force density

For the linear elliptic equation arising in linear elasticity, the residual $\mathcal{R}_{\mathfrak{m}}^{\text {lin }} \in \mathcal{H}^{*}$ is given by

$$
\begin{aligned}
\left\langle\mathcal{R}_{\mathfrak{m}}^{\text {lin }}, \boldsymbol{\varphi}\right\rangle_{-1,1} & :=\langle\boldsymbol{f}, \boldsymbol{\varphi}\rangle+\langle\boldsymbol{\pi}, \boldsymbol{\varphi}\rangle_{\Gamma_{N}}-a\left(\boldsymbol{u}_{\mathfrak{m}}, \boldsymbol{\varphi}\right) \\
& =a\left(\boldsymbol{u}-\boldsymbol{u}_{\mathfrak{m}}, \boldsymbol{\varphi}\right)
\end{aligned}
$$

and we call it linear residual. From the properties of the bilinear form $a(\cdot, \cdot)$ it follows that the error in the $H^{1}$-norm $\left\|\boldsymbol{u}-\boldsymbol{u}_{\mathfrak{m}}\right\|_{1}$ is bounded by the norm of the residual

$$
\left\|\boldsymbol{u}-\boldsymbol{u}_{\mathfrak{m}}\right\|_{1} \lesssim\left\|\mathcal{R}_{\mathfrak{m}}^{\text {lin }}\right\|_{-1} .
$$

and the norm of the residual is bounded by the error

$$
\left\|\mathcal{R}_{\mathfrak{m}}^{\operatorname{lin}}\right\|_{-1, \omega} \lesssim\left\|\boldsymbol{u}-\boldsymbol{u}_{\mathfrak{m}}\right\|_{1, \omega} .
$$

for each open subset $\omega$ of $\Omega$. The dual norm $\left\|\mathcal{R}_{\mathfrak{m}}^{\text {lin }}\right\|_{-1}$ is not a computable quantity. However, it is bounded from below and above by computable quantities as the element residual $\boldsymbol{r}\left(\boldsymbol{u}_{\mathfrak{m}}\right):=\operatorname{div} \boldsymbol{\sigma}\left(\boldsymbol{u}_{\mathfrak{m}}\right)+\boldsymbol{f}$ and the jump terms which are either the difference between the stresses $\left.\boldsymbol{\sigma}\right|_{\mathfrak{e}}\left(\boldsymbol{u}_{\mathfrak{m}}\right) \cdot \boldsymbol{n}$ and $\left.\boldsymbol{\sigma}\right|_{\mathfrak{e}}\left(\boldsymbol{u}_{\mathfrak{m}}\right) \cdot \boldsymbol{n}$ of two neighboring elements $\mathfrak{e}$ and $\tilde{\mathfrak{e}}$ where $\boldsymbol{n}$ is the unit outward normal to the common side $\mathfrak{s}$ in the interior of $\Omega$, or the difference between the given Neumann data $\boldsymbol{\pi}$ and the boundary stress $\left.\hat{\boldsymbol{\sigma}}\right|_{\mathfrak{e}}\left(\boldsymbol{u}_{\mathfrak{m}}\right)$ at a Neumann boundary side $\mathfrak{s}$

$$
\begin{aligned}
\boldsymbol{J}^{I}\left(\boldsymbol{u}_{\mathfrak{m}}\right) & :=\left(\left.\boldsymbol{\sigma}\right|_{\mathfrak{e}}\left(\boldsymbol{u}_{\mathfrak{m}}\right)-\left.\boldsymbol{\sigma}\right|_{\mathfrak{e}}\left(\boldsymbol{u}_{\mathfrak{m}}\right)\right) \boldsymbol{n} \\
\boldsymbol{J}^{N}\left(\boldsymbol{u}_{\mathfrak{m}}\right) & :=\boldsymbol{\pi}-\left.\hat{\boldsymbol{\sigma}}\right|_{\mathfrak{e}}\left(\boldsymbol{u}_{\mathfrak{m}}\right) .
\end{aligned}
$$

The relation $\left\langle\mathcal{R}_{\mathfrak{m}}^{\operatorname{lin}}, \boldsymbol{\varphi}\right\rangle_{-1,1}=a\left(\boldsymbol{u}-\boldsymbol{u}_{\mathfrak{m}}, \boldsymbol{\varphi}\right)$, compare (8), is disturbed in the case of contact problems

$$
a\left(\boldsymbol{u}-\boldsymbol{u}_{\mathfrak{m}}, \boldsymbol{\varphi}\right)=\left\langle\mathcal{R}_{\mathfrak{m}}^{\text {lin }}, \boldsymbol{\varphi}\right\rangle_{-1,1}-\langle\boldsymbol{\lambda}, \boldsymbol{\varphi}\rangle_{-1,1} \quad \forall \boldsymbol{\varphi} \in \mathcal{H}_{0}
$$

In contrast to the linear elliptic case, the linear residual is no appropriate measure for the error because it is non-zero if continuous and discrete solution coincide, thus, it overestimates the error. The contact force density $\boldsymbol{\lambda}$ as well as the displacements $\boldsymbol{u}$ are both unknowns of the contact problem. Considering this structure the first reliable and efficient residual-type a posteriori error estimator for the closely related obstacle problem has been presented in [31]. Therein the error is measured in both unknowns, in the displacements and the constraining force density. Accordingly, the linear residual is replaced by a Galerkin functional whose abstract definition, in the case of contact problems, is given by

$$
\begin{aligned}
\left\langle\mathcal{G}_{\mathfrak{m}}, \boldsymbol{\varphi}\right\rangle_{-1,1} & :=a\left(\boldsymbol{u}-\boldsymbol{u}_{\mathfrak{m}}, \boldsymbol{\varphi}\right)+\left\langle\boldsymbol{\lambda}-\tilde{\boldsymbol{\lambda}}_{\mathfrak{m}}, \boldsymbol{\varphi}\right\rangle_{-1,1} \\
& =\left\langle\mathcal{R}_{\mathfrak{m}}^{\operatorname{lin}}-\tilde{\boldsymbol{\lambda}}_{\mathfrak{m}}, \boldsymbol{\varphi}\right\rangle_{-1,1} \quad \forall \boldsymbol{\varphi} \in \mathcal{H}_{0}
\end{aligned}
$$

where $\tilde{\lambda}_{\mathfrak{m}}$, still to be specified, is an approximation of $\boldsymbol{\lambda}_{\mathfrak{m}}$ for discrete test functions $\boldsymbol{\varphi}_{\mathfrak{m}} \in$ $\mathcal{H}_{\mathfrak{m}, 0}$ and is additionally defined for non-discrete test functions $\varphi \in \mathcal{H}_{0}$. Notice that if $\left\langle\tilde{\lambda}_{\mathfrak{m}}, \boldsymbol{\varphi}_{\mathfrak{m}}\right\rangle_{-1,1}=\left\langle\boldsymbol{\lambda}_{\mathfrak{m}}, \boldsymbol{\varphi}_{\mathfrak{m}}\right\rangle_{-1,1}$, then (13) generalizes the Galerkin orthogonality in that $\left\langle\mathcal{G}_{\mathfrak{m}}, \boldsymbol{\varphi}_{\mathfrak{m}}\right\rangle_{-1,1}=0$. Moreover, following [6], $\mathcal{G}_{\mathfrak{m}}$ may be seen as the linear residual of an auxiliary equation. For obstacle problems different choices of $\tilde{\lambda}_{\mathfrak{m}}$ have been proposed in [12, 
24-26,31]. We refer to [35] for a discussion about residual-type a posteriori error estimators for obstacle and contact problems.

In this section we define a $\tilde{\lambda}_{\mathfrak{m}}$ which depends on the discrete solution and data and reflects the properties of $\boldsymbol{\lambda}$ as, e.g., in [12,24]. We call it quasi-discrete contact force density. To this end, we need some preliminary definitions. The patch $\omega_{p}$ is the interior of the union of all surrounding elements. The corresponding diameter is abbreviated with $h_{p}:=\operatorname{diam} \omega_{p}$ and the union of all sides of elements belonging to $\bar{\omega}_{p}$ is denoted by $\gamma_{p}$. We call the union of all sides in the interior of $\omega_{p}$, not including the boundary of $\omega_{p}$, skeleton and denote it by $\gamma_{p, I}$. Given any $\boldsymbol{p} \in N_{\mathfrak{m}}^{C}$, we subdivide the intersections between $\Gamma$ and $\partial \omega_{p}$ in the three following sets:

$$
\begin{aligned}
\gamma_{p, C} & :=\Gamma_{C} \cap \partial \omega_{p}, \\
\gamma_{p, N} & :=\Gamma_{N} \cap \partial \omega_{p}, \\
\gamma_{p, D} & :=\Gamma_{D} \cap \partial \omega_{p} .
\end{aligned}
$$

For the ease of presentation, we choose the coordinate system such that $\boldsymbol{e}_{1}=\boldsymbol{\nu}$. Hence, the discrete admissible set (7) is given by

$$
\mathcal{K}_{\mathfrak{m}}=\left\{\boldsymbol{v}_{\mathfrak{m}} \in \mathcal{H}_{\mathfrak{m}} \mid v_{\mathfrak{m}, 1} \leq g_{\mathfrak{m}} \text { on } \Gamma_{C}\right\}
$$

In the following, we make use of the representation

$$
\langle\boldsymbol{\lambda}, \boldsymbol{\varphi}\rangle_{-1,1}=\sum_{i=1}^{d}\left\langle\lambda_{i}, \varphi_{i}\right\rangle_{-1,1}
$$

of the contact force density with the components

$$
\begin{aligned}
\left\langle\lambda_{1}, \varphi_{1}\right\rangle_{-1,1} & :=-\left\langle\hat{\sigma}_{1}(\boldsymbol{u}), \varphi_{1}\right\rangle_{-\frac{1}{2}, \frac{1}{2}, \Gamma_{C}} \\
\left\langle\lambda_{i}, \varphi_{i}\right\rangle_{-1,1} & :=0, \quad i \in\{2, . ., d\}
\end{aligned}
$$

where we exploited that the frictional stresses are zero.

The discrete contact force density coincides with the linear residual as functional on $\mathcal{H}_{\mathfrak{m}, 0}$,

$$
\left\langle\boldsymbol{\lambda}_{\mathfrak{m}}, \boldsymbol{\varphi}_{\mathfrak{m}}\right\rangle_{-1,1}:=\left\langle\boldsymbol{f}, \boldsymbol{\varphi}_{\mathfrak{m}}\right\rangle-a\left(\boldsymbol{u}_{\mathfrak{m}}, \boldsymbol{\varphi}_{\mathfrak{m}}\right)+\left\langle\boldsymbol{\pi}, \boldsymbol{\varphi}_{\mathfrak{m}}\right\rangle_{\Gamma_{N}}=\left\langle\mathcal{R}_{\mathfrak{m}}^{\operatorname{lin}}, \boldsymbol{\varphi}_{\mathfrak{m}}\right\rangle_{-1,1} \quad \forall \boldsymbol{\varphi}_{\mathfrak{m}} \in \mathcal{H}_{\mathfrak{m}, 0}
$$

In order to investigate $\boldsymbol{\lambda}_{\mathfrak{m}}$ further, we use integration by parts

$$
\begin{aligned}
& \left\langle\boldsymbol{\lambda}_{\mathfrak{m}}, \boldsymbol{\varphi}_{\mathfrak{m}}\right\rangle_{-1,1} \\
& =\sum_{i=1}^{d} \sum_{p \in N_{\mathfrak{m}}}\left\langle\boldsymbol{\lambda}_{\mathfrak{m}}, \varphi_{\mathfrak{m}, i}(\boldsymbol{p}) \phi_{p} \boldsymbol{e}_{i}\right\rangle_{-1,1} \\
& =\sum_{i=1}^{d} \sum_{p \in N_{\mathfrak{m}}} \int_{\gamma_{p, I}} \boldsymbol{J}^{I}\left(\boldsymbol{u}_{\mathfrak{m}}\right) \cdot \varphi_{\mathfrak{m}, i}(\boldsymbol{p}) \phi_{p} \boldsymbol{e}_{i}+\sum_{i=1}^{d} \sum_{p \in N_{\mathfrak{m}}} \int_{\omega_{p}} \boldsymbol{r}\left(\boldsymbol{u}_{\mathfrak{m}}\right) \cdot \varphi_{\mathfrak{m}, i}(\boldsymbol{p}) \phi_{p} \boldsymbol{e}_{i} \\
& \quad+\sum_{i=1}^{d} \sum_{p \in N_{\mathfrak{m}}^{N}} \int_{\gamma_{p}, N} \boldsymbol{J}^{N}\left(\boldsymbol{u}_{\mathfrak{m}}\right) \cdot \varphi_{\mathfrak{m}, i}(\boldsymbol{p}) \phi_{p} \boldsymbol{e}_{i}-\sum_{i=1}^{d} \sum_{p \in N_{\mathfrak{m}}^{C}} \int_{\gamma_{p, C}} \hat{\boldsymbol{\sigma}}\left(\boldsymbol{u}_{\mathfrak{m}}\right) \cdot \varphi_{\mathfrak{m}, i}(\boldsymbol{p}) \phi_{p} \boldsymbol{e}_{i}
\end{aligned}
$$


for all $\varphi_{\mathfrak{m}} \in \mathcal{H}_{\mathfrak{m}, 0}$. Here, we use the notation $N_{\mathfrak{m}}^{\bar{N}}$ for the set of all nodes on $\bar{\Gamma}_{N}$. As constraints are solely imposed in direction $e_{1}$, homogeneous Neumann boundary conditions hold in the other directions at the potential contact boundary. Therefore, we define $\boldsymbol{J}_{T}^{C}\left(\boldsymbol{u}_{\mathfrak{m}}\right):=-\hat{\boldsymbol{\sigma}}_{T}\left(\boldsymbol{u}_{\mathfrak{m}}\right)$.

We recall that the constraints are only imposed at contact nodes $N_{\mathfrak{m}}^{C}$ in direction $e_{1}$, so that (16) reduces to

$$
\begin{aligned}
\left\langle\boldsymbol{\lambda}_{\mathfrak{m}}, \boldsymbol{\varphi}_{\mathfrak{m}}\right\rangle_{-1,1}= & \sum_{p \in N_{\mathfrak{m}}^{C}} \int_{\gamma_{p, I}} J_{1}^{I}\left(\boldsymbol{u}_{\mathfrak{m}}\right) \cdot \varphi_{\mathfrak{m}, 1}(\boldsymbol{p}) \phi_{p}+\sum_{p \in N_{\mathfrak{m}}^{C}} \int_{\omega_{p}} r_{1}\left(\boldsymbol{u}_{\mathfrak{m}}\right) \cdot \varphi_{\mathfrak{m}, 1}(\boldsymbol{p}) \phi_{p} \\
& +\sum_{p \in N_{\mathfrak{m}}^{C}} \int_{\gamma_{p, N}} J_{1}^{N}\left(\boldsymbol{u}_{\mathfrak{m}}\right) \cdot \varphi_{\mathfrak{m}, 1}(\boldsymbol{p}) \phi_{p}-\sum_{p \in N_{\mathfrak{m}}^{C}} \int_{\gamma_{p}, C} \hat{\sigma}_{1}\left(\boldsymbol{u}_{\mathfrak{m}}\right) \cdot \varphi_{\mathfrak{m}, 1}(\boldsymbol{p}) \phi_{p}
\end{aligned}
$$

which motivates the representation

$$
\left\langle\boldsymbol{\lambda}_{\mathfrak{m}}, \boldsymbol{\varphi}_{\mathfrak{m}}\right\rangle_{-1,1}=\sum_{i=1}^{d}\left\langle\lambda_{\mathfrak{m}, i}, \varphi_{\mathfrak{m}, i}\right\rangle_{-1,1}
$$

with

$$
\begin{aligned}
\left\langle\lambda_{\mathfrak{m}, 1}, \varphi_{\mathfrak{m}, 1}\right\rangle_{-1,1} & :=\left\langle\lambda_{\mathfrak{m}}, \varphi_{\mathfrak{m}}\right\rangle_{-1,1} \\
\left\langle\lambda_{\mathfrak{m}, i}, \varphi_{\mathfrak{m}, i}\right\rangle_{-1,1} & :=0, \quad i \in\{2, . ., d\} .
\end{aligned}
$$

We mention that $\left\langle\mathcal{R}_{\mathfrak{m}}^{\text {lin }}, \phi_{p} \boldsymbol{e}_{1}\right\rangle_{-1,1}=\left\langle\lambda_{\mathfrak{m}, 1}, \phi_{p}\right\rangle_{-1,1} \geq 0$, which follows from the discrete variational inequality. Notice that identity (17) is the discrete counterpart of $\langle\boldsymbol{\lambda}, \boldsymbol{\varphi}\rangle_{-1,1}=$ $-\left\langle\hat{\sigma}_{1}(\boldsymbol{u}), \varphi_{1}\right\rangle_{-\frac{1}{2}, \frac{1}{2}, \Gamma_{C}}$. Hence in the discrete setting the relation between the contact force density and the contact stresses is polluted by element and jump residuals. This is due to the fact that nodal values at the contact boundary affect the displacements at the boundary as well as in the interior. The quasi-discrete contact force density $\tilde{\boldsymbol{\lambda}}_{\mathfrak{m}}$ should be related to a functional on $\Gamma_{C}$.

In order to prepare the definition of $\tilde{\lambda}_{\mathfrak{m}}$, we classify the actual contact nodes $\boldsymbol{p} \in N_{\mathfrak{m}}^{C}$ with $u_{\mathfrak{m}, 1}(\boldsymbol{p})=g_{\mathfrak{m}}(\boldsymbol{p})$ in two different categories. At so-called full-contact nodes $\boldsymbol{p} \in N_{\mathfrak{m}}^{\mathrm{fC}}$ the discrete solution fulfills the following conditions

- $u_{\mathfrak{m}, 1}=g_{\mathfrak{m}} \quad$ on $\gamma_{p, C}$

- $\hat{\sigma}_{1}\left(\boldsymbol{u}_{\mathfrak{m}}\right) \leq 0 \quad$ on $\gamma_{p, C}$

which means that the conditions of actual contact of Problem 1 are satisfied. The remaining actual contact nodes are called semi-contact nodes and the set is denoted by $N_{\mathfrak{m}}^{\mathrm{sC}}$.

The following definition of $\tilde{\boldsymbol{\lambda}}_{\mathfrak{m}}$ uses this classification by means of a partition of unity

$$
\left\langle\tilde{\lambda}_{\mathfrak{m}}, \varphi\right\rangle_{-1,1}:=\left\langle\tilde{\lambda}_{\mathfrak{m}, 1}, \varphi_{1}\right\rangle_{-1,1}:=\sum_{p \in N_{\mathfrak{m}}^{C}}\left\langle\tilde{\lambda}_{\mathfrak{m}, 1}^{p}, \varphi_{1} \phi_{p}\right\rangle_{-1,1}
$$

and adjusts the local contributions so that, on one hand, the Galerkin functional is prepared for the derivation of an upper bound, and on the other hand, tries to maximize the cancellation within $\mathcal{G}_{\mathfrak{m}}$ thanks to $\tilde{\boldsymbol{\lambda}}_{\mathfrak{m}}$.

For semi-contact nodes $\boldsymbol{p} \in N_{\mathfrak{m}}^{\mathrm{sC}}$, we define

$$
\left\langle\tilde{\lambda}_{\mathfrak{m}, 1}^{p}, \varphi_{1} \phi_{p}\right\rangle_{-1,1}:=\left\langle\mathcal{R}_{\mathfrak{m}, 1}^{\operatorname{lin}}, \phi_{p}\right\rangle_{-1,1} c_{p}\left(\varphi_{1}\right)=s_{p} c_{p}\left(\varphi_{1}\right) \int_{\gamma_{p, C}} \phi_{p}
$$


where $s_{p}:=\frac{\left\langle\lambda_{\mathfrak{m}, 1}, \phi_{p}\right\rangle_{-1,1}}{\int_{\gamma_{p}, C} \phi_{p}}=\frac{\left\langle\mathcal{R}_{\mathfrak{m}, 1}^{\operatorname{lin}}, \phi_{p}\right\rangle_{-1,1}}{\int_{\gamma_{p}, C} \phi_{p}}$ is the nodal value of the discrete contact force density obtained by lumping the boundary mass matrix and $c_{p}\left(\varphi_{1}\right)$ is defined below.

For full-contact nodes $\boldsymbol{p} \in N_{\mathfrak{m}}^{\mathrm{fC}}$, we define

$$
\begin{aligned}
\left\langle\tilde{\lambda}_{\mathfrak{m}, 1}^{p}, \varphi_{1} \phi_{p}\right\rangle_{-1,1} & :=\left\langle\mathcal{R}_{\mathfrak{m}, 1}, \phi_{p}\right\rangle_{-1,1} c_{p}\left(\varphi_{1}\right)-\int_{\gamma_{p, C}} \hat{\sigma}_{1}\left(\boldsymbol{u}_{\mathfrak{m}}\right) \varphi_{1} \phi_{p} \\
& =s_{p} c_{p}\left(\varphi_{1}\right) \int_{\gamma_{p, C}} \phi_{p}-\int_{\gamma_{p}, C} \hat{\sigma}_{1}\left(\boldsymbol{u}_{\mathfrak{m}}\right)\left(\varphi_{1}-c_{p}\left(\varphi_{1}\right)\right) \phi_{p}
\end{aligned}
$$

where

$$
\left\langle\mathcal{R}_{\mathfrak{m}, 1}, \varphi_{1}\right\rangle_{-1,1}:=\left\langle\mathcal{R}_{\mathfrak{m}, 1}^{\operatorname{lin}}, \varphi_{1}\right\rangle_{-1,1}+\int_{\Gamma_{C}} \hat{\sigma}_{1}\left(\boldsymbol{u}_{\mathfrak{m}}\right) \varphi_{1}
$$

For nodes which are no actual contact nodes we may also use the definition (19) of $\left\langle\tilde{\lambda}_{\mathfrak{m}, 1}^{p}, \varphi_{1} \phi_{p}\right\rangle_{-1,1}$ because $s_{p}=0$ if $u_{\mathfrak{m}}(\boldsymbol{p}) \neq g_{\mathfrak{m}}(\boldsymbol{p})$.

It will turn out that in order to prove an upper and lower bound of the error measure

$$
\left\|\boldsymbol{u}-\boldsymbol{u}_{\mathfrak{m}}\right\|_{1}+\left\|\boldsymbol{\lambda}-\tilde{\boldsymbol{\lambda}}_{\mathfrak{m}}\right\|_{-1}
$$

we need specific choices of $c_{p}(\varphi)$ for semi- and full-contact nodes. Here and in the following, elements $\mathfrak{e} \in \mathfrak{m}$ and sides $\mathfrak{s} \in \mathfrak{m}$ are understood as subdomains of $\Omega$, too.

For full-contact nodes $\boldsymbol{p} \in N_{\mathfrak{m}}^{\mathrm{fC}}$, we use

$$
c_{p}\left(\varphi_{1}\right):=\max _{\mathfrak{s} \subset \gamma_{p}, C} \frac{\int_{\mathfrak{s}} \varphi_{1} \phi_{p}}{\int_{\mathfrak{s}} \phi_{p}} .
$$

This choice is important for the derivation of the upper bound, see Section 4.2 and 4.3, respectively.

For semi-contact nodes $\boldsymbol{p} \in N_{\mathfrak{m}}^{\mathrm{sC}}$, we take

$$
c_{p}\left(\varphi_{1}\right)=\frac{\int_{\tilde{\gamma}_{p, C}} \varphi_{1} \phi_{p}}{\int_{\tilde{\gamma}_{p, C}} \phi_{p}}
$$

where $\tilde{\gamma}_{p, C}$ is a strict subset of $\gamma_{p, C}$, such that for every two different nodes $\boldsymbol{p}_{1} \in \gamma_{p, C}$ and $\boldsymbol{p}_{2} \in \gamma_{p, C}, \tilde{\gamma}_{p_{1}, C} \cap \tilde{\gamma}_{p_{2}, C}=\emptyset$. The use of $\tilde{\gamma}_{p, C}$ instead of $\gamma_{p, C}$ will be exploited for the derivation of lower bounds, see Section 5.1.

We note that we can choose any of the above examples of $c_{p}(\varphi)$ for nodes $\boldsymbol{p} \in N_{\mathfrak{m}}^{C}$ which are no actual contact nodes.

To our best knowledge the definition of $\tilde{\lambda}_{\mathfrak{m}}$ is new. Corresponding a priori error estimates are beyond the scope of this article.

3.2 Error estimator and main results

The error estimator

$$
\eta:=\sum_{i=1}^{9} \eta_{i}
$$


for which we prove efficiency and reliability in the following sections consists of the following contributions:

$$
\begin{aligned}
& \eta_{1}:=\left(\sum_{p \in N_{\mathfrak{m}}} \eta_{1, p}^{2}\right)^{\frac{1}{2}}, \quad \eta_{1, p}:=h_{p}\left\|\boldsymbol{r}\left(\boldsymbol{u}_{\mathfrak{m}}\right)\right\|_{\omega_{p}} \\
& \eta_{2}:=\left(\sum_{p \in N_{\mathfrak{m}}} \eta_{2, p}^{2}\right)^{\frac{1}{2}}, \quad \eta_{2, p}:=h_{p}^{\frac{1}{2}}\left\|\boldsymbol{J}^{I}\left(\boldsymbol{u}_{\mathfrak{m}}\right)\right\|_{\gamma_{p, I}} \\
& \eta_{3}:=\left(\sum_{p \in N_{\mathfrak{m}}^{\bar{N}}} \eta_{3, p}^{2}\right)^{\frac{1}{2}}, \quad \eta_{3, p}:=h_{p}^{\frac{1}{2}}\left\|\boldsymbol{J}^{N}\left(\boldsymbol{u}_{\mathfrak{m}}\right)\right\|_{\gamma_{p, N}} \\
& \eta_{4}:=\left(\sum_{p \in N_{\mathfrak{m}}^{C}} \eta_{4, p}^{2}\right)^{\frac{1}{2}}, \quad \eta_{4, p}:=h_{p}^{\frac{1}{2}}\left\|\boldsymbol{J}_{T}^{C}\left(\boldsymbol{u}_{\mathfrak{m}}\right)\right\|_{\gamma_{p, C}} \\
& \eta_{5}:=\left(\sum_{p \in N_{\mathfrak{m}}^{C} \backslash N_{\mathfrak{m}}^{\mathrm{fC}}} \eta_{5, p}^{2}\right)^{\frac{1}{2}}, \quad \eta_{5, p}:=h_{p}^{\frac{1}{2}}\left\|\hat{\sigma}_{1}\left(\boldsymbol{u}_{\mathfrak{m}}\right)\right\|_{\gamma_{p, C}} \\
& \eta_{6}:=\left(\sum_{p \in N_{\mathfrak{m}}^{\mathrm{sC}}} \eta_{6, p}^{2}\right)^{\frac{1}{2}}, \quad \eta_{6, p}:=\left(s_{p} d_{p}\right)^{\frac{1}{2}} \\
& \eta_{7}:=\left(\sum_{p \in N_{\mathfrak{m}}^{\mathrm{sC}} \cup N_{\mathfrak{m}}^{\mathrm{fC}}} \eta_{7, p}^{2}\right)^{\frac{1}{2}} \\
& \eta_{7, p}:=\left\{\begin{array}{c}
\left(s_{p} \int_{\gamma_{p, C}} \phi_{p} \frac{\int_{\tilde{\gamma}_{p, C}}\left(g-g_{\mathfrak{m}}\right)^{+} \phi_{p}}{\int_{\tilde{\gamma}_{p}, C} \phi_{p}}\right)^{\frac{1}{2}} \text { if } \boldsymbol{p} \in N_{\mathfrak{m}}^{\mathrm{sC}} \\
\left(s_{p} \int_{\gamma_{p, C}} \phi_{p}\left(\sum_{\mathfrak{s} \subset \gamma_{p, C}} \frac{\int_{\mathfrak{s}}\left(g-g_{\mathfrak{m}}\right)^{+} \phi_{p}}{\int_{\mathfrak{s}} \phi_{p}}+\frac{\int_{\mathfrak{s}}\left(g_{\mathfrak{m}}-g\right)^{+} \phi_{p}}{\int_{\mathfrak{s}} \phi_{p}}\right)\right)^{\frac{1}{2}} \text { if } \boldsymbol{p} \in N_{\mathfrak{m}}^{\mathrm{fC}}
\end{array}\right. \\
& \eta_{8}:=\left\|\left(u_{\mathfrak{m}, 1}-g\right)^{+}\right\|_{\frac{1}{2}, \Gamma_{C}}, \quad \eta_{8, p}:=\left\|\left(u_{\mathfrak{m}, 1}-g\right)^{+} \phi_{p}\right\|_{\frac{1}{2}, \gamma_{p, C}} .
\end{aligned}
$$

Here and in the following we denote the positive part of a function by $\varphi^{+}:=\max \{\varphi, 0\}$ and the negative part by $\varphi^{-}:=\max \{-\varphi, 0\}$ such that $\varphi=\varphi^{+}-\varphi^{-}$. The abbreviation $d_{p}$ in $\eta_{6}$ stands for

$$
d_{p}:=\int_{\tilde{\gamma}_{p, C}}\left(g_{\mathfrak{m}}-u_{\mathfrak{m}, 1}\right) \phi_{p}
$$

where $\tilde{\gamma}_{p, C}$ is a strict subset of $\gamma_{p, C}$. Thus, $\eta_{6}$ reminds of a complementarity condition. In fact, for a semi-contact node $s_{p} d_{p}$ would be a complementarity condition with respect to the quasi-discrete contact force density $\left\langle\tilde{\lambda}_{\mathfrak{m}, 1}^{p},\left(g_{\mathfrak{m}}-u_{\mathfrak{m}, 1}\right) \phi_{p}\right\rangle_{-1,1}$ if $\tilde{\gamma}_{p, C}$ was replaced by $\gamma_{p, C}$. We refer to $\eta_{6}$ as complementarity residual and call $\eta_{5}$ contact stress residual. The contributions $\eta_{5}, \eta_{6}$ are localized to semi-contact nodes and nodes which are not actually 
in contact. Further we call $\eta_{7}$ obstacle approximation and $\eta_{8}$ actual constraint violation. In the absence of any contact, we have $\eta_{6}=\eta_{7}=\eta_{8}=0$ and $\eta_{5}$ has contributions from all potential contact nodes such that $\eta$ is a residual error estimator for linear elliptic boundary value problems where the potential contact boundary is replaced by a Neumann boundary with $\pi=0$. If contact occurs such an error estimator for linear equations would overestimate the error because the expected boundary stresses in the actual contact zone are non-zero. The contributions $\eta_{7}, \eta_{8}$ deal with the case $g \neq g_{\mathfrak{m}}$. The different structure of $\eta_{7, p}$ and $\eta_{8, p}$ is due to the fact that the difference between $u_{\mathfrak{m}, 1}$ and $g$ is computable while the difference between $u_{1}$ and $g_{\mathfrak{m}}$ is not known.

The main results about reliability and efficiency of the error estimator $\eta$ defined in (22) and its local contributions are stated in the following theorems and are proven in Sections 4 and 5. We assume the direction of constraints $\nu$ to be constant and we consider meshes of simplices. The exact solution of Problem 2 is denoted by $\boldsymbol{u}$ and the finite element solution of Problem 3 by $\boldsymbol{u}_{\mathfrak{m}}$. The continuous contact force density is given by $\boldsymbol{\lambda}$ and the quasi-discrete contact force density $\tilde{\lambda}_{\mathfrak{m}}$ is defined as in Section 3.1. Further let $\bar{f}$ and $\bar{\pi}$ be piecewise constant approximations of $\boldsymbol{f}$ and $\boldsymbol{\pi}$.

Theorem 1 Reliability of the error estimator

The error estimator $\eta$ provides an upper bound of the error measure (21):

$$
\left\|\boldsymbol{u}-\boldsymbol{u}_{\mathfrak{m}}\right\|_{1}+\left\|\boldsymbol{\lambda}-\tilde{\boldsymbol{\lambda}}_{\mathfrak{m}}\right\|_{-1} \lesssim \eta
$$

Theorem 2 Local lower bounds

The local lower bounds

$$
\eta_{k, p} \lesssim\left\|\boldsymbol{u}-\boldsymbol{u}_{\mathfrak{m}}\right\|_{1, \omega_{p}}+\left\|\boldsymbol{\lambda}-\tilde{\boldsymbol{\lambda}}_{\mathfrak{m}}\right\|_{-1, \omega_{p}}+h_{p}\|\overline{\boldsymbol{f}}-\boldsymbol{f}\|_{\omega_{p}}+h_{p}^{\frac{1}{2}}\|\overline{\boldsymbol{\pi}}-\boldsymbol{\pi}\|_{\gamma_{p, N}}
$$

hold for $k=1,2$ with $\boldsymbol{p} \in N_{\mathfrak{m}}$, for $k=3$ with $\boldsymbol{p} \in N_{\mathfrak{m}}^{\bar{N}}$, for $k=4$ with $\boldsymbol{p} \in N_{\mathfrak{m}}^{C}$ and for $k=5$ with $\boldsymbol{p} \in N_{\mathfrak{m}}^{C} \backslash N_{\mathfrak{m}}^{\mathrm{fC}}$.

Under the assumption that each $\boldsymbol{p} \in N_{\mathfrak{m}}^{\mathrm{sC}}$ has a neighboring interior node $\boldsymbol{p} \in N_{\mathfrak{m}}^{I}$ and for a suitable extension $\bar{g}_{\mathfrak{m}} \in \mathcal{H}_{\mathfrak{m}}$ of $g_{\mathfrak{m}}$ to a finite element function on $\Omega$, the following estimate holds for $\eta_{6, p}$ :

$$
\eta_{6, p} \lesssim\left\|\boldsymbol{u}-\boldsymbol{u}_{\mathfrak{m}}\right\|_{1, \omega_{p}}+\left\|\boldsymbol{\lambda}-\tilde{\boldsymbol{\lambda}}_{\mathfrak{m}}\right\|_{-1, \omega_{p}}+h_{p}\|\overline{\boldsymbol{f}}-\boldsymbol{f}\|_{\omega_{p}}+h_{p}^{\frac{1}{2}}\left\|\boldsymbol{J}\left(\begin{array}{c}
\bar{g}_{\mathfrak{m}} \\
0
\end{array}\right)\right\|_{\gamma_{p, C}}
$$

where for simplicity we supposed that the actual contact zone is a strict subset of the potential contact boundary.

We recall that $h_{p}\|\overline{\boldsymbol{f}}-\boldsymbol{f}\|_{\omega_{p}}$ and $h_{p}^{\frac{1}{2}}\|\overline{\boldsymbol{\pi}}-\boldsymbol{\pi}\|_{\gamma_{p, N}}$ are formally of higher order. We do not provide lower bounds in terms of the additional error estimator parts $\eta_{7, p}, \eta_{8, p}, \eta_{9, p}$, but notice that their counterparts for obstacle problems cannot be neglected in the upper bound because all the other error estimator contributions might be zero, while the real problem is not solved due to $g_{\mathfrak{m}} \neq g$, compare Examples 4.1 and 4.2 in [31].

\section{Reliability of the error estimator}

In this section we give the proof of Theorem 1. Our starting point is that the error in the contact force densities $\left\|\boldsymbol{\lambda}-\tilde{\boldsymbol{\lambda}}_{\mathfrak{m}}\right\|_{-1}$ is bounded by means of $\left\|\mathcal{G}_{\mathfrak{m}}\right\|_{-1}$ and $\left\|\boldsymbol{u}-\boldsymbol{u}_{\mathfrak{m}}\right\|_{1}$. This 
follows from the definition of the Galerkin functional, the boundedness of the bilinear form, i.e., $a(\varphi, \tilde{\varphi}) \leq\|\varphi\|_{1}\|\tilde{\varphi}\|_{1}$ for all $\varphi, \tilde{\varphi} \in \mathcal{H}$ and Young's inequality:

$$
\left\|\boldsymbol{\lambda}-\tilde{\boldsymbol{\lambda}}_{\mathfrak{m}}\right\|_{-1}^{2} \lesssim\left\|\mathcal{G}_{\mathfrak{m}}\right\|_{-1}^{2}+\left\|\boldsymbol{u}-\boldsymbol{u}_{\mathfrak{m}}\right\|_{1}^{2}
$$

If we evaluate the Galerkin functional at $\boldsymbol{u}-\boldsymbol{u}_{\mathfrak{m}} \in \mathcal{H}_{0}$ and exploit the ellipticity of the bilinear form $a(\cdot, \cdot)$ on $\mathcal{H}_{0}$, we obtain

$$
\left\|\boldsymbol{u}-\boldsymbol{u}_{\mathfrak{m}}\right\|_{1}^{2} \lesssim\left\|\mathcal{G}_{\mathfrak{m}}\right\|_{-1}^{2}+2\left\langle\tilde{\boldsymbol{\lambda}}_{\mathfrak{m}}-\boldsymbol{\lambda}, \boldsymbol{u}-\boldsymbol{u}_{\mathfrak{m}}\right\rangle_{-1,1} .
$$

Thus, the error in the displacements is bounded by the dual norm of the Galerkin functional and a duality pairing between the contact force densities and the displacements. In Section 4.1 we show that $\left\|\mathcal{G}_{\mathfrak{m}}\right\|_{-1}$ is bounded by the error estimator and we deal with the second term in Sections 4.2 and 4.3 depending on the kind of gap function.

\subsection{Upper bound of the Galerkin functional}

For the proof of the upper bound of $\left\|\mathcal{G}_{\mathfrak{m}}\right\|_{-1}$, we rewrite $\left\langle\mathcal{G}_{\mathfrak{m}}, \boldsymbol{\varphi}\right\rangle_{-1,1}$ as follows:

$$
\begin{aligned}
& \left\langle\mathcal{G}_{\mathfrak{m}}, \varphi\right\rangle_{-1,1} \\
& =a\left(\boldsymbol{u}-\boldsymbol{u}_{\mathfrak{m}}, \boldsymbol{\varphi}\right)+\left\langle\boldsymbol{\lambda}-\tilde{\boldsymbol{\lambda}}_{\mathfrak{m}}, \boldsymbol{\varphi}\right\rangle_{-1,1} \\
& =\langle\boldsymbol{f}, \boldsymbol{\varphi}\rangle+\langle\boldsymbol{\pi}, \boldsymbol{\varphi}\rangle_{\Gamma_{N}}-a\left(\boldsymbol{u}_{\mathfrak{m}}, \boldsymbol{\varphi}\right)-\left\langle\tilde{\boldsymbol{\lambda}}_{\mathfrak{m}}, \boldsymbol{\varphi}\right\rangle_{-1,1} \\
& =\sum_{i=1}^{d} \sum_{p \in N_{\mathfrak{m}} \backslash N_{\mathfrak{m}}^{C}}\left\langle\mathcal{R}_{\mathfrak{m}, i}^{\operatorname{lin}}, \varphi_{i} \phi_{p}\right\rangle_{-1,1}+\sum_{i=2}^{d} \sum_{p \in N_{\mathfrak{m}}^{C}}\left\langle\mathcal{R}_{\mathfrak{m}, i}^{\operatorname{lin}}, \varphi_{i} \phi_{p}\right\rangle_{-1,1} \\
& +\sum_{p \in N_{\mathfrak{m}}^{C}}\left(\left\langle\mathcal{R}_{\mathfrak{m}, 1}, \varphi_{1} \phi_{p}\right\rangle_{-1,1}-\int_{\Gamma_{C}} \hat{\sigma}_{1}\left(\boldsymbol{u}_{\mathfrak{m}}\right) \varphi_{1} \phi_{p}-\left\langle\tilde{\lambda}_{\mathfrak{m}, 1}^{p}, \varphi_{1} \phi_{p}\right\rangle_{-1,1}\right) \\
& =\sum_{i=1}^{d} \sum_{p \in N_{\mathfrak{m}} \backslash N_{\mathfrak{m}}^{C}}\left\langle\mathcal{R}_{\mathfrak{m}, i}^{\operatorname{lin}},\left(\varphi_{i}-c_{p}\left(\varphi_{i}\right)\right) \phi_{p}\right\rangle_{-1,1}+\sum_{i=2}^{d} \sum_{p \in N_{\mathfrak{m}}^{C}}\left\langle\mathcal{R}_{\mathfrak{m}, i}^{\operatorname{lin}},\left(\varphi_{i}-c_{p}\left(\varphi_{i}\right)\right) \phi_{p}\right\rangle_{-1,1} \\
& +\sum_{p \in N_{\mathfrak{m}}^{C}}\left\langle\mathcal{R}_{\mathfrak{m}, 1},\left(\varphi_{1}-c_{p}\left(\varphi_{1}\right)\right) \phi_{p}\right\rangle_{-1,1}-\sum_{p \in N_{\mathfrak{m}}^{C} \backslash N_{\mathfrak{m}}^{\mathrm{fC}}} \int_{\Gamma_{C}} \hat{\sigma}_{1}\left(\boldsymbol{u}_{\mathfrak{m}}\right)\left(\varphi_{1}-c_{p}\left(\varphi_{1}\right)\right) \phi_{p} .
\end{aligned}
$$

Here we set $c_{p}\left(\varphi_{i}\right)=0$ for Dirichlet nodes. We exploited $\left\langle\mathcal{R}_{\mathfrak{m}, i}^{\text {lin }}, \phi_{p}\right\rangle_{-1,1}=0$ for all noncontact nodes and for $i=2, . ., d$ at contact nodes and we inserted the definition of $\tilde{\lambda}_{\mathfrak{m}}$. For all $\boldsymbol{p} \in N_{\mathfrak{m}} \backslash\left(N_{\mathfrak{m}}^{D} \cup N_{\mathfrak{m}}^{C}\right)$ and for all contact nodes with $i \neq 1$ we choose the constants

$$
c_{p}\left(\varphi_{i}\right):=\frac{\int_{\omega_{p}} \varphi_{i} \phi_{p}}{\int_{\omega_{p}} \phi_{p}} .
$$

The mean values (27) fulfill the standard $L^{2}$-approximation properties

$$
\begin{gathered}
\left\|\varphi-c_{p}(\varphi)\right\|_{\omega_{p}} \lesssim h_{p}\|\nabla \varphi\|_{\omega_{p}} \\
\left\|\varphi-c_{p}(\varphi)\right\|_{\gamma_{p}} \lesssim h_{p}^{\frac{1}{2}}\|\nabla \varphi\|_{\omega_{p}},
\end{gathered}
$$


e.g., [32]. For Dirichlet nodes we have at least one edge or side $\mathfrak{s} \subset \gamma_{p, D}$, where the test function $\varphi$ is zero, therefore we can deduce $\|\varphi\|_{\omega_{p}} \lesssim h_{p}\|\nabla \varphi\|_{\omega_{p}}$ directly from the PoincaréFriedrichs inequality. The $L^{2}$-approximation properties hold also for the constants $c_{p}(\varphi)$ defined in Section 3.1 for semi- and full-contact nodes, see [29].

Lemma 1 The Galerkin functional with the quasi-discrete contact force density of Section 3.1 satisfies

$$
\left\|\mathcal{G}_{\mathfrak{m}}\right\|_{-1} \lesssim\left(\sum_{k=1}^{5} \eta_{k}^{2}\right)^{\frac{1}{2}}
$$

Proof Inserting the definition of $\mathcal{R}_{\mathfrak{m}, i}^{\operatorname{lin}}$ and $\mathcal{R}_{\mathrm{m}, i}$ in (26), we get

$$
\begin{aligned}
\left\langle\mathcal{G}_{\mathfrak{m}}, \boldsymbol{\varphi}\right\rangle_{-1,1} & \sum_{i=1}^{d} \sum_{p \in N_{\mathfrak{m}}}\left(\int_{\gamma_{p, I}} J_{i}^{I}\left(\boldsymbol{u}_{\mathfrak{m}}\right)\left(\varphi_{i}-c_{p}\left(\varphi_{i}\right)\right) \phi_{p}+\int_{\omega_{p}} r_{i}\left(\boldsymbol{u}_{\mathfrak{m}}\right)\left(\varphi_{i}-c_{p}\left(\varphi_{i}\right)\right) \phi_{p}\right) \\
& +\sum_{i=1}^{d} \sum_{p \in N_{\mathfrak{m}}^{N}} \int_{\gamma_{p, N}} J_{i}^{N}\left(\boldsymbol{u}_{\mathfrak{m}}\right)\left(\varphi_{i}-c_{p}\left(\varphi_{i}\right)\right) \phi_{p} \\
& +\sum_{i=2}^{d} \sum_{p \in N_{\mathfrak{m}}^{C}} \int_{\gamma_{p}, C} J_{i}^{C}\left(\boldsymbol{u}_{\mathfrak{m}}\right)\left(\varphi_{i}-c_{p}\left(\varphi_{i}\right)\right) \phi_{p} \\
& -\sum_{p \in N_{\mathfrak{m}}^{C} \backslash N_{\mathfrak{m}}^{\mathrm{fC}}} \int_{\Gamma_{C}} \hat{\sigma}_{1}\left(\boldsymbol{u}_{\mathfrak{m}}\right)\left(\varphi_{1}-c_{p}\left(\varphi_{1}\right)\right) \phi_{p} \\
\lesssim & \sum_{i=1}^{d} \sum_{p \in N_{\mathfrak{m}}}\left(h_{p}^{\frac{1}{2}}\left\|J_{i}^{I}\left(\boldsymbol{u}_{\mathfrak{m}}\right)\right\|_{\gamma_{p}, I}\left\|\nabla \varphi_{i}\right\|_{\gamma_{p}, I}+h_{p}\left\|r_{i}\left(\boldsymbol{u}_{\mathfrak{m}}\right)\right\|_{\omega_{p}}\left\|\nabla \varphi_{i}\right\|_{\omega_{p}}\right) \\
& +\sum_{i=1}^{d} \sum_{p \in N_{\mathfrak{m}}^{N}}\left(h_{p}^{\frac{1}{2}}\left\|J_{i}^{N}\left(\boldsymbol{u}_{\mathfrak{m}}\right)\right\|_{\gamma_{p}, N}\left\|\nabla \varphi_{i}\right\|_{\gamma_{p}, N}\right) \\
& +\sum_{i=2}^{d} \sum_{p \in N_{\mathfrak{m}}^{C}}\left(h_{p}^{\frac{1}{2}}\left\|J_{i}^{C}\left(\boldsymbol{u}_{\mathfrak{m}}\right)\right\|_{\gamma_{p, C}}\left\|\nabla \varphi_{i}\right\|_{\gamma_{p}, C}\right) \\
& +\sum_{p \in N_{\mathfrak{m}}^{C} \backslash N_{\mathfrak{m}}^{\mathrm{fC}}}\left(h_{p}^{\frac{1}{2}}\left\|\hat{\sigma}_{1}\left(\boldsymbol{u}_{\mathfrak{m}}\right)\right\|_{\gamma_{p}, C}\left\|\nabla \varphi_{1}\right\|_{\gamma_{p}, C}\right) \\
\lesssim & \left(\sum_{k=1}^{5} \eta_{k}^{2}\right)^{\frac{1}{2}}\|\boldsymbol{\varphi}\|_{1, \Omega}
\end{aligned}
$$

where we applied Hölder's inequality and the $L^{2}$-approximation properties and we exploited the shape regularity.

\subsection{Upper bound in the case of a discrete gap function}

As we have already proven the upper bound of $\left\|\mathcal{G}_{\mathfrak{m}}\right\|_{-1}$ it remains to bound $\left\langle\tilde{\boldsymbol{\lambda}}_{\mathfrak{m}}-\boldsymbol{\lambda}, \boldsymbol{u}-\boldsymbol{u}_{\mathfrak{m}}\right\rangle_{-1,1}$. We have $\left\langle\lambda_{i}, \varphi_{i}\right\rangle=\left\langle\tilde{\lambda}_{\mathfrak{m}, i}, \varphi_{i}\right\rangle=0$ for $i \neq 1$ and $\left\langle\lambda_{1}, u_{\mathfrak{m}, 1}-u_{1}\right\rangle_{-1,1} \leq 0$ because $\mathcal{K}_{\mathfrak{m}} \subset \mathcal{K}$ 
as long as $g_{\mathfrak{m}}=g$. Thus, it remains to bound $\left\langle\tilde{\lambda}_{\mathfrak{m}, 1}, u_{1}-u_{\mathfrak{m}, 1}\right\rangle_{-1,1}$. From the definition of the quasi-discrete contact force density $\tilde{\lambda}_{\mathfrak{m}, 1}(18,20,19)$ follows

$$
\begin{aligned}
\left\langle\tilde{\lambda}_{\mathfrak{m}, 1}, u_{1}-u_{\mathfrak{m}, 1}\right\rangle_{-1,1}= & \sum_{p \in N_{\mathfrak{m}}^{\mathrm{sC}}}\left(s_{p} c_{p}\left(u_{1}-u_{\mathfrak{m}, 1}\right) \int_{\gamma_{p}, C} \phi_{p}\right) \\
& +\sum_{p \in N_{\mathfrak{m}}^{\mathrm{fC}}}\left(s_{p} c_{p}\left(u_{1}-u_{\mathfrak{m}, 1}\right) \int_{\gamma_{p}, C} \phi_{p}\right) \\
& -\sum_{p \in N_{\mathfrak{m}}^{\mathrm{fC}}}\left(\int_{\gamma_{p}, C} \hat{\sigma}_{1}\left(\boldsymbol{u}_{\mathfrak{m}}\right)\left(u_{1}-u_{\mathfrak{m}, 1}-c_{p}\left(u_{1}-u_{\mathfrak{m}, 1}\right)\right) \phi_{p}\right) .
\end{aligned}
$$

The first sum in (30) is bounded by $\eta_{6}$

$$
\begin{aligned}
& \sum_{p \in N_{\mathfrak{m}}^{\mathrm{sC}}} s_{p} \int_{\gamma_{p, C}} \phi_{p} \frac{\int_{\tilde{\gamma}_{p, C}}\left(u_{1}-u_{\mathfrak{m}, 1}\right) \phi_{p}}{\int_{\tilde{\gamma}_{p, C}} \phi_{p}} \\
= & \sum_{p \in N_{\mathfrak{m}}^{\mathrm{sC}}}\left(s_{p} \int_{\gamma_{p, C}} \phi_{p} \frac{\int_{\tilde{\gamma}_{p, C}}\left(u_{1}-g\right) \phi_{p}}{\int_{\tilde{\gamma}_{p, C}} \phi_{p}}+s_{p} \int_{\gamma_{p, C}} \phi_{p} \frac{\int_{\tilde{\gamma}_{p, C}}\left(g-u_{\mathfrak{m}, 1}\right) \phi_{p}}{\int_{\tilde{\gamma}_{p, C}} \phi_{p}}\right) \\
\lesssim & \sum_{p \in N_{\mathfrak{m}}^{\mathrm{sC}}} s_{p} d_{p}
\end{aligned}
$$

where we exploit $s_{p} \geq 0$ for $p \in N_{\mathfrak{m}}^{\mathrm{C}}, u_{1} \leq g=g_{\mathfrak{m}}$ and $\frac{\int_{\gamma_{p, C}} \phi_{p}}{\int_{\tilde{\gamma}_{p}, C} \phi_{p}}$ is a constant independent of $h_{p}$ if $\tilde{\gamma}_{p, C}$ is always a fixed fraction of $\gamma_{p, C}$.

For a full-contact node $\boldsymbol{p}$, we have $u_{1} \leq g=g_{\mathfrak{m}}=u_{\mathfrak{m}, 1}$ which implies $u_{1}-u_{\mathfrak{m}, 1} \leq 0$ and, therefore, $c_{p}\left(u_{1}-u_{\mathfrak{m}, 1}\right) \leq 0$. As further $s_{p} \geq 0$ we have $s_{p} c_{p}\left(u_{1}-u_{\mathfrak{m}, 1}\right) \leq 0$ such that the second sum is bounded by zero. In order to estimate the third sum we decompose it in a sum of integrals over sides. We exploit $\hat{\sigma}_{1}\left(\boldsymbol{u}_{\mathfrak{m}}\right)$ constant on each side as we assumed meshes of simplices and $c_{p}\left(u_{1}-u_{\mathfrak{m}, 1}\right) \geq \frac{\int_{\mathfrak{s}}\left(u_{1}-u_{\mathfrak{m}, 1}\right) \phi_{p}}{\int_{\mathfrak{s}} \phi_{p}}$ for each side $\mathfrak{s}$ in $\gamma_{p, C}$ to obtain

$$
\begin{aligned}
& -\sum_{p \in N_{\mathfrak{m}}^{\mathrm{fC}}} \int_{\gamma_{p, C}} \hat{\sigma}_{1}\left(\boldsymbol{u}_{\mathfrak{m}}\right)\left(u_{1}-u_{\mathfrak{m}, 1}-c_{p}\left(u_{1}-u_{\mathfrak{m}, 1}\right)\right) \phi_{p} \\
& \quad=\sum_{p \in N_{\mathfrak{m}}^{\mathrm{fC}}} \sum_{\mathfrak{s} \subset \gamma_{p}, C}-\left.\hat{\sigma}_{1}\left(\boldsymbol{u}_{\mathfrak{m}}\right)\right|_{\mathfrak{s}} \underbrace{\int_{\mathfrak{s}}\left(u_{1}-u_{\mathfrak{m}, 1}-c_{p}\left(u_{1}-u_{\mathfrak{m}, 1}\right)\right) \phi_{p}}_{\leq 0} \leq 0 .
\end{aligned}
$$

This contribution is also bounded by zero. Thus, we have

$$
\left\langle\tilde{\boldsymbol{\lambda}}_{\mathfrak{m}}-\boldsymbol{\lambda}, \boldsymbol{u}-\boldsymbol{u}_{\mathfrak{m}}\right\rangle_{-1,1} \lesssim \eta_{6}^{2}=\sum_{p \in N_{\mathfrak{m}}^{\mathrm{sC}}} s_{p} d_{p}
$$

Putting together (24), (25), (29) and (32) we have the proof of Theorem 1 if $g=g_{\mathfrak{m}}$.

4.3 Upper bound for a general gap function in $H^{\frac{1}{2}}$

In the foregoing section we used the fact, that $u_{\mathfrak{m}, 1} \leq g$ if $g_{\mathfrak{m}}=g$ in order to get an upper bound of $\left\langle\tilde{\boldsymbol{\lambda}}_{\mathfrak{m}}-\boldsymbol{\lambda}, \boldsymbol{u}-\boldsymbol{u}_{\mathfrak{m}}\right\rangle_{-1,1}$. This condition may not hold for an arbitrary function $g \in$ 
$H^{\frac{1}{2}}\left(\Gamma_{C}\right)$. However, it is possible to give an upper bound of the error measure by means of the error estimator for arbitrary gap functions. For this purpose, we define a function

$$
u_{\mathfrak{m}, 1}^{*}=\min \left\{\left.u_{\mathfrak{m}, 1}\right|_{\Gamma_{C}}, g\right\} \in H^{\frac{1}{2}}\left(\Gamma_{C}\right)
$$

and a harmonic extension $\tilde{w}$ of $w:=u_{\mathfrak{m}, 1}-u_{\mathfrak{m}, 1}^{*} \in H^{\frac{1}{2}}\left(\Gamma_{C}\right)$ so that the stability estimate (see e.g., [30], pp. 70-71)

$$
\left\|u_{\mathfrak{m}, 1}-u_{\mathfrak{m}, 1}^{*}\right\|_{1} \lesssim\left\|u_{\mathfrak{m}, 1}-u_{\mathfrak{m}, 1}^{*}\right\|_{\frac{1}{2}, \Gamma_{C}}
$$

holds. We set $u_{\mathfrak{m}, 1}^{*}:=u_{\mathfrak{m}, 1}-\tilde{w} \in \mathcal{H}$. In a first step we find an upper bound of $\left\langle-\lambda_{1}, u_{1}-u_{\mathfrak{m}, 1}\right\rangle$. To this end, we use $\left\langle\lambda_{1}, u_{\mathfrak{m}, 1}^{*}-u_{1}\right\rangle_{-1,1} \leq 0$ as $u_{\mathfrak{m}, 1}^{*} \leq g$, Young's inequality and (33) to derive

$$
\begin{aligned}
& \left\langle-\lambda_{1}, u_{1}-u_{\mathfrak{m}, 1}\right\rangle_{-1,1} \\
& =\left\langle\lambda_{1}, u_{\mathfrak{m}, 1}-u_{\mathfrak{m}, 1}^{*}+u_{\mathfrak{m}, 1}^{*}-u_{1}\right\rangle_{-1,1} \\
& \leq\left\langle\lambda_{1}, u_{\mathfrak{m}, 1}-u_{\mathfrak{m}, 1}^{*}\right\rangle_{-1,1} \\
& =\left\langle\lambda_{1}-\tilde{\lambda}_{\mathfrak{m}, 1}, u_{\mathfrak{m}, 1}-u_{\mathfrak{m}, 1}^{*}\right\rangle_{-1,1}+\left\langle\tilde{\lambda}_{\mathfrak{m}, 1}, u_{\mathfrak{m}, 1}-u_{\mathfrak{m}, 1}^{*}\right\rangle_{-1,1} \\
& \leq \frac{1}{2}\left\|\lambda_{1}-\tilde{\lambda}_{\mathfrak{m}, 1}\right\|_{-1}^{2}+\frac{1}{2}\left\|u_{\mathfrak{m}, 1}-u_{\mathfrak{m}, 1}^{*}\right\|_{1}^{2}+\left\langle\tilde{\lambda}_{\mathfrak{m}, 1}, u_{\mathfrak{m}, 1}-u_{\mathfrak{m}, 1}^{*}\right\rangle_{-1,1} \\
& \lesssim \frac{1}{2}\left\|\lambda_{1}-\tilde{\lambda}_{\mathfrak{m}, 1}\right\|_{-1}^{2}+\frac{1}{2}\left\|u_{\mathfrak{m}, 1}-u_{\mathfrak{m}, 1}^{*}\right\|_{\frac{1}{2}, \Gamma_{C}}^{2}+\left\langle\tilde{\lambda}_{\mathfrak{m}, 1}, u_{\mathfrak{m}, 1}-u_{\mathfrak{m}, 1}^{*}\right\rangle_{-1,1}
\end{aligned}
$$

Therefrom we deduce

$$
\begin{aligned}
\left\langle\tilde{\lambda}_{\mathfrak{m}, 1}-\lambda_{1}, u_{1}-u_{\mathfrak{m}, 1}\right\rangle_{-1,1} & \lesssim \frac{1}{2}\left\|\lambda_{1}-\tilde{\lambda}_{\mathfrak{m}, 1}\right\|_{-1}^{2}+\frac{1}{2}\left\|u_{\mathfrak{m}, 1}-u_{\mathfrak{m}, 1}^{*}\right\|_{\frac{1}{2}, \Gamma_{C}}^{2} \\
& +\left\langle\tilde{\lambda}_{\mathfrak{m}, 1}, u_{1}-u_{\mathfrak{m}, 1}\right\rangle_{-1,1}+\left\langle\tilde{\lambda}_{\mathfrak{m}, 1}, u_{\mathfrak{m}, 1}-u_{\mathfrak{m}, 1}^{*}\right\rangle_{-1,1}
\end{aligned}
$$


An upper bound of the last two terms of (34) is given by

$$
\begin{aligned}
&\left\langle\tilde{\lambda}_{\mathfrak{m}, 1}, u_{1}-u_{\mathfrak{m}, 1}\right\rangle_{-1,1}+\left\langle\tilde{\lambda}_{\mathfrak{m}, 1}, u_{\mathfrak{m}, 1}-u_{\mathfrak{m}, 1}^{*}\right\rangle_{-1,1} \\
&=\sum_{p \in N_{\mathfrak{m}}^{C}}\left\langle\tilde{\lambda}_{\mathfrak{m}, 1}^{p},\left(u_{1}-u_{\mathfrak{m}, 1}\right) \phi_{p}\right\rangle_{-1,1}+\sum_{p \in N_{\mathfrak{m}}^{C}}\left\langle\tilde{\lambda}_{\mathfrak{m}, 1}^{p},\left(u_{\mathfrak{m}, 1}-g\right)^{+} \phi_{p}\right\rangle_{-1,1} \\
&=\sum_{p \in N_{\mathfrak{m}}^{s C}}\left(s_{p} c_{p}\left(u_{1}-u_{\mathfrak{m}, 1}\right) \int_{\gamma_{p}, C} \phi_{p}\right)+\sum_{p \in N_{\mathfrak{m}}^{s C}}\left(s_{p} c_{p}\left(\left(u_{\mathfrak{m}, 1}-g\right)^{+}\right) \int_{\gamma_{p}, C} \phi_{p}\right) \\
& \quad+\sum_{p \in N_{\mathfrak{m}}^{\mathrm{fC}}}\left(s_{p} c_{p}\left(u_{1}-u_{\mathfrak{m}, 1}\right) \int_{\gamma_{p}, C} \phi_{p}\right)+\sum_{p \in N_{\mathfrak{m}}^{\mathrm{fC}}}\left(s_{p} c_{p}\left(\left(u_{\mathfrak{m}, 1}-g\right)^{+}\right) \int_{\gamma_{p}, C} \phi_{p}\right) \\
&-\sum_{p \in N_{\mathfrak{m}}^{\mathrm{fC}}}\left(\int_{\gamma_{p}, C} \hat{\sigma}_{1}\left(\boldsymbol{u}_{\mathfrak{m}}\right)\left(\left(u_{1}-u_{\mathfrak{m}, 1}\right)-c_{p}\left(u_{1}-u_{\mathfrak{m}, 1}\right)\right) \phi_{p}\right) \\
&-\underbrace{}_{p \in N_{\mathfrak{m}}^{\mathrm{fC}}}\left(\int_{\gamma_{p}, C} \hat{\sigma}_{1}\left(\boldsymbol{u}_{\mathfrak{m}}\right)\left(\left(u_{\mathfrak{m}, 1}-g\right)^{+}-c_{p}\left(\left(u_{\mathfrak{m}, 1}-g\right)^{+}\right)\right) \phi_{p}\right) \\
& \leq \sum_{p \in N_{\mathfrak{m}}^{\mathrm{sC}}}\left(s_{p} c_{p}\left(\left(g-u_{\mathfrak{m}, 1}\right)^{+}\right) \int_{\gamma_{p}, C} \phi_{p}\right) \\
&+\sum_{p \in N_{\mathfrak{m}}^{\mathrm{fC}}}\left(s_{p} c_{p}\left(u_{1}-u_{\mathfrak{m}, 1}\right) \int_{\gamma_{p}, C} \phi_{p}\right)+\sum_{p \in N_{\mathfrak{m}}^{\mathrm{fC}}}\left(s_{p} c_{p}\left(\left(u_{\mathfrak{m}, 1}-g\right)^{+}\right) \int_{\gamma_{p}, C} \phi_{p}\right)
\end{aligned}
$$

where we exploited the relation

$$
\left.\left(u_{\mathfrak{m}, 1}-u_{\mathfrak{m}, 1}^{*}\right)\right|_{\Gamma_{C}}=\left.\left(u_{\mathfrak{m}, 1}-g\right)^{+}\right|_{\Gamma_{C}},
$$

the definition of $\tilde{\lambda}_{\mathfrak{m}, 1}$ and for semi-contact nodes

$$
\begin{aligned}
& \left(u_{1}-u_{\mathfrak{m}, 1}\right)+\left(u_{\mathfrak{m}, 1}-g\right)^{+} \\
& =\left(u_{1}-g\right)+\left(g-u_{\mathfrak{m}, 1}\right)+\left(u_{\mathfrak{m}, 1}-g\right)^{+} \\
& \leq\left(g-u_{\mathfrak{m}, 1}\right)+\left(u_{\mathfrak{m}, 1}-g\right)^{+} \\
& =\left(u_{\mathfrak{m}, 1}-g\right)^{-}=\left(g-u_{\mathfrak{m}, 1}\right)^{+} .
\end{aligned}
$$

In the case of semi-contact nodes we further exploit that $\left(g_{\mathfrak{m}}-u_{\mathfrak{m}, 1}\right)=\left(g_{\mathfrak{m}}-u_{\mathfrak{m}, 1}\right)^{+}$so that

$\left(g-u_{\mathfrak{m}, 1}\right)^{+}=\left(g-u_{\mathfrak{m}, 1}+g_{\mathfrak{m}}-g_{\mathfrak{m}}\right)^{+} \leq\left(g_{\mathfrak{m}}-u_{\mathfrak{m}, 1}\right)^{+}+\left(g-g_{\mathfrak{m}}\right)^{+}=\left(g_{\mathfrak{m}}-u_{\mathfrak{m}, 1}\right)+\left(g-g_{\mathfrak{m}}\right)^{+}$.

Thus, we have for the first sum in (35)

$$
\begin{aligned}
& \sum_{p \in N_{\mathfrak{m}}^{\mathrm{sC}}}\left(s_{p} c_{p}\left(\left(g-u_{\mathfrak{m}, 1}\right)^{+}\right) \int_{\gamma_{p}, C} \phi_{p}\right) \\
\lesssim & \sum_{p \in N_{\mathfrak{m}}^{\mathrm{sC}}} s_{p} d_{p}+\sum_{p \in N_{\mathfrak{m}}^{\mathrm{sC}}}\left(s_{p} c_{p}\left(\left(g-g_{\mathfrak{m}}\right)^{+}\right) \int_{\gamma_{p}, C} \phi_{p}\right) \\
\lesssim & \eta_{6}^{2}+\sum_{p \in N_{\mathfrak{m}}^{\mathrm{sC}}} \eta_{7, p}^{2} .
\end{aligned}
$$


For the reformulation of the second sum in (35) we have a closer look at the mean value $c_{p}\left(u_{1}-u_{\mathfrak{m}, 1}\right)$ for full-contact nodes. Let $S^{*}$ be the side of $\gamma_{p, C}$ fulfilling $c_{p}\left(u_{1}-u_{\mathfrak{m}, 1}\right)=$ $\frac{\int_{S^{*}}\left(u_{1}-u_{\mathfrak{m}, 1}\right) \phi_{p}}{\int_{S^{*}} \phi_{p}}$. As $u_{1} \leq g$ and $g-u_{\mathfrak{m}, 1}=g-g_{\mathfrak{m}}$ for full-contact nodes we get

$$
\begin{aligned}
c_{p}\left(u_{1}-u_{\mathfrak{m}, 1}\right) & \leq \frac{\int_{S^{*}}\left(g-u_{\mathfrak{m}, 1}\right) \phi_{p}}{\int_{S^{*}} \phi_{p}} \\
& \leq \frac{\int_{S^{*}}\left(g-g_{\mathfrak{m}}\right) \phi_{p}}{\int_{S^{*}} \phi_{p}} \\
& \leq \frac{\int_{S^{*}}\left(g-g_{\mathfrak{m}}\right)^{+} \phi_{p}}{\int_{S^{*}} \phi_{p}} \\
& \leq \sum_{S \subset \gamma_{p}, C} \frac{\int_{S}\left(g-g_{\mathfrak{m}}\right)^{+} \phi_{p}}{\int_{S} \phi_{p}}
\end{aligned}
$$

In the third term of (35) we may replace $u_{\mathfrak{m}, 1}$ by $g_{\mathfrak{m}}$ as full-contact nodes are considered. Thus, the second and the third sum in (35) is bounded by $\sum_{p \in N_{\mathfrak{m}}^{\mathrm{fC}}} \eta_{7, p}^{2}$.

$$
\begin{aligned}
& \sum_{p \in N_{\mathfrak{m}}^{\mathrm{fC}}}\left(s_{p} c_{p}\left(u_{1}-u_{\mathfrak{m}, 1}\right) \int_{\gamma_{p}, C} \phi_{p}\right)+\sum_{p \in N_{\mathfrak{m}}^{\mathrm{fC}}}\left(s_{p} c_{p}\left(\left(u_{\mathfrak{m}, 1}-g\right)^{+}\right) \int_{\gamma_{p}, C} \phi_{p}\right) \\
\leq & \sum_{p \in N_{\mathfrak{m}}^{\mathrm{fC}}}\left(s_{p} \int_{\gamma_{p}, C} \phi_{p}\left(\sum_{\mathfrak{s} \subset \gamma_{p}, C} \frac{\int_{\mathfrak{s}}\left(g-g_{\mathfrak{m}}\right)^{+} \phi_{p}}{\int_{\mathfrak{s}} \phi_{p}}+\frac{\int_{\mathfrak{s}}\left(g_{\mathfrak{m}}-g\right)^{+} \phi_{p}}{\int_{\mathfrak{s}} \phi_{p}}\right)\right) \\
= & \eta_{7}^{2}
\end{aligned}
$$

Putting together $(35,36,37,38,39,40)$, (34) is bounded by

$$
\begin{aligned}
\left\langle\tilde{\lambda}_{\mathfrak{m}, 1}-\lambda_{1}, u_{1}-u_{\mathfrak{m}, 1}\right\rangle_{-1,1} & \lesssim \frac{1}{2}\left\|\lambda_{1}-\tilde{\lambda}_{\mathfrak{m}, 1}\right\|_{-1}^{2}+\frac{1}{2}\left\|u_{\mathfrak{m}, 1}-u_{\mathfrak{m}, 1}^{*}\right\|_{\frac{1}{2}, \Gamma_{C}}^{2} \\
& +\left\langle\tilde{\lambda}_{\mathfrak{m}, 1}, u_{1}-u_{\mathfrak{m}, 1}\right\rangle_{-1,1}+\left\langle\tilde{\lambda}_{\mathfrak{m}, 1}, u_{\mathfrak{m}, 1}-u_{\mathfrak{m}, 1}^{*}\right\rangle_{-1,1} \\
& \lesssim \frac{1}{2}\left\|\lambda_{1}-\tilde{\lambda}_{\mathfrak{m}, 1}\right\|_{-1}^{2}+\frac{1}{2}\left\|\left(u_{\mathfrak{m}, 1}-g\right)^{+}\right\|_{\frac{1}{2}, \Gamma_{C}}^{2}+\eta_{6}^{2}+\eta_{7}^{2} \\
& \lesssim \frac{1}{2}\left\|\lambda_{1}-\tilde{\lambda}_{\mathfrak{m}, 1}\right\|_{-1}^{2}+\eta_{6}^{2}+\eta_{7}^{2}+\eta_{8}^{2} .
\end{aligned}
$$

Together with (24), (25), (29) we have the proof of Theorem 1 if $g \in H^{\frac{1}{2}}\left(\Gamma_{C}\right)$.

\section{Efficiency of the error estimator}

In this section we give the proof of Theorem 2. The proof of the lower bounds in terms of the contributions $\eta_{1, p}, \ldots, \eta_{4, p}$

$$
\sum_{k=1}^{4} \eta_{k, p} \lesssim\left\|\boldsymbol{u}-\boldsymbol{u}_{\mathfrak{m}}\right\|_{1, \omega_{p}}+\left\|\boldsymbol{\lambda}-\tilde{\boldsymbol{\lambda}}_{\mathfrak{m}}\right\|_{-1, \omega_{p}}+h_{p}\|\overline{\boldsymbol{f}}-\boldsymbol{f}\|_{\omega_{p}}+h_{p}^{\frac{1}{2}}\|\overline{\boldsymbol{\pi}}-\boldsymbol{\pi}\|_{\gamma_{p, N}}
$$

follows like in the case of a linear elliptic problem. In fact $\mathcal{G}_{\mathfrak{m}}$ plays the role of $\mathcal{R}_{\mathfrak{m}}^{\text {lin }}$. The properties of the bubble functions, see, e.g., [33] are used and the boundedness of the Galerkin functional by means of the error measure

$$
\left\|\mathcal{G}_{\mathfrak{m}}\right\|_{-1, \omega_{p}} \leq\left\|\boldsymbol{u}-\boldsymbol{u}_{\mathfrak{m}}\right\|_{1, \omega_{p}}+\left\|\boldsymbol{\lambda}-\tilde{\boldsymbol{\lambda}}_{\mathfrak{m}}\right\|_{-1, \omega_{p}}
$$


which follows directly from the definition of the Galerkin functional. The proofs that the local contributions $\eta_{5, p}$ and $\eta_{6, p}$ provide local lower bounds of the error measure are more intricate and will be given in detail in this section.

5.1 Lower bound in terms of the contribution $\eta_{5, p}$

In this section we show that $\eta_{5, p}$ is a lower bound of the error for all nodes $\boldsymbol{p} \in N_{\mathfrak{m}}^{C} \backslash N_{\mathfrak{m}}^{f C}$. Similar to the case of $\eta_{1, p}, \eta_{2, p}, \eta_{3, p}, \eta_{4, p}$ we make use of the relation between the Galerkin functional and the quantity of interest which here is the boundary stress.

Let $\overline{\boldsymbol{p}} \in N_{\mathfrak{m}}^{C} \backslash N_{\mathfrak{m}}^{\mathrm{fC}}$ be an arbitrary but fixed node. In the following, $\mathfrak{s}$ denotes a side which belongs to $\gamma_{\bar{p}, C}$. We take the corresponding side bubble function

$$
\Psi_{\mathfrak{s}}:=\prod_{p \in \mathfrak{s}} \phi_{p}
$$

as test function in (26)

$$
\begin{aligned}
& \sum_{p \in N_{\mathfrak{m}}^{C} \backslash N_{\mathfrak{m}}^{\mathrm{fC}}} \int_{\Gamma_{C}} \hat{\sigma}_{1}\left(\boldsymbol{u}_{\mathfrak{m}}\right) \Psi_{\mathfrak{s}} \phi_{p} \\
= & -\left\langle\mathcal{G}_{\mathfrak{m}}, \Psi_{\mathfrak{s}} \boldsymbol{e}_{1}\right\rangle_{-1,1}+\sum_{p \in N_{\mathfrak{m}} \backslash N_{\mathfrak{m}}^{C}}\left\langle\mathcal{R}_{\mathfrak{m}, 1}^{\operatorname{lin}}, \Psi_{\mathfrak{s}} \phi_{p}\right\rangle_{-1,1} \\
& +\sum_{p \in N_{\mathfrak{m}}^{C}}\left\langle\mathcal{R}_{\mathfrak{m}, 1},\left(\Psi_{\mathfrak{s}}-c_{p}\left(\Psi_{\mathfrak{s}}\right)\right) \phi_{p}\right\rangle_{-1,1}+\sum_{p \in N_{\mathfrak{m}}^{C} \backslash N_{\mathfrak{m}}^{\mathrm{fC}}} \int_{\Gamma_{C}} \hat{\sigma}_{1}\left(\boldsymbol{u}_{\mathfrak{m}}\right) c_{p}\left(\Psi_{\mathfrak{s}}\right) \phi_{p} \\
= & -\left\langle\mathcal{G}_{\mathfrak{m}}, \Psi_{\mathfrak{s}} e_{1}\right\rangle_{-1,1}+\sum_{p \in N_{\mathfrak{m}}} \int_{\omega_{\mathfrak{s}}} r_{1}\left(\boldsymbol{u}_{\mathfrak{m}}\right) \Psi_{\mathfrak{s}} \phi_{p} \\
& -\sum_{p \in N_{\mathfrak{m}}^{\mathrm{sC}}} s_{p} c_{p}\left(\Psi_{\mathfrak{s}}\right) \int_{\gamma_{p, C}} \phi_{p}-\sum_{p \in N_{\mathfrak{m}}^{\mathrm{fC}}}\left\langle\mathcal{R}_{\mathfrak{m}, 1}, \phi_{p}\right\rangle c_{p}\left(\Psi_{\mathfrak{s}}\right) .
\end{aligned}
$$

If the side $\mathfrak{s}$ is not contained in any patch $\gamma_{p, C}$ of semi- or full-contact nodes $\boldsymbol{p}$, the two last terms are zero and we can proceed similar to the case of $\eta_{1, p}, \eta_{2, p}, \eta_{3, p}, \eta_{4, p}$. Otherwise, in order to get rid of the last two terms, we replace $\Psi_{\mathfrak{s}}$ by a suitable function $\theta_{\mathfrak{s}}$ such that in addition $c_{p}\left(\theta_{\mathfrak{s}}\right)=0$ for all semi- and full-contact nodes. The value $c_{p}(\cdot)$ for a semi-contact node $\boldsymbol{p}$ depends on $\tilde{\gamma}_{p, C}$ which is a strict subset of $\gamma_{p, C}$, compare Section 3.1. If $\gamma_{p, C}$ consists of two intervals we choose the inner third of $\gamma_{p, C}$ containing $\boldsymbol{p}$ as $\tilde{\gamma}_{p, C}$. If $\gamma_{p, C}$ is a union of triangles we refine each triangle ones uniformly and generate $\tilde{\gamma}_{p, C}$ as the $2 D$ patch enclosing $\boldsymbol{p}$ with respect to this subgrid. For example in Figure 1, the dark blue region is $\tilde{\gamma}_{p, C}$ for $\boldsymbol{p}=\boldsymbol{p}_{1}$. A side $\mathfrak{s}$ has $d$ nodes $\left\{\boldsymbol{p}_{i}\right\}_{i=1, \ldots, d}$. We denote the sides of the subgrid containing $\boldsymbol{p}_{i}$ by $\mathfrak{s}_{i}$ and the middle part by $\mathfrak{s}_{M}$, see Figure 1 . For the function $\theta_{\mathfrak{s}}$ we make the following ansatz

$$
\theta_{\mathfrak{s}}=\left(\sum_{i=1}^{d} a_{i} \Psi_{i}+a_{M} \Psi_{M}\right)
$$

where $\Psi_{i}$ and $\Psi_{M}$ are side bubble functions to $\mathfrak{s}_{i}$ and $\mathfrak{s}_{M}$. The coefficients are determined so that

1. $\int_{\mathfrak{s}} 1=\sum_{p \in N_{\mathfrak{m}} \backslash N_{\mathfrak{m}}^{\mathrm{fC}}} \int_{\mathfrak{s}} \theta_{\mathfrak{s}} \phi_{p}$

2. $\int_{\mathfrak{s}_{i}} \theta_{\mathfrak{s}} \phi_{p_{i}}=0$ for all $\boldsymbol{p}_{i}$ semi-contact nodes 


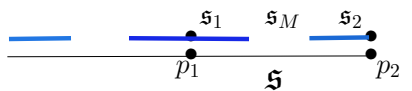

(a) Subgrid of an interval

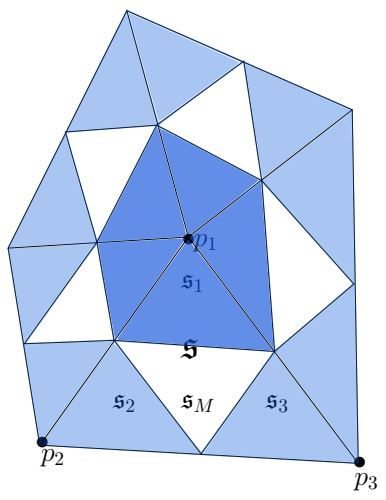

(b) Subgrid of a triangle

Fig. 1: Subgrid of boundary patches $\gamma_{p, C}$

3. $\int_{\mathfrak{s}} \theta_{\mathfrak{s}} \phi_{p_{i}}=0$ for all $\boldsymbol{p}_{i}$ full-contact nodes with $\mathfrak{s}=\overline{\mathfrak{s}}$.

As $\bar{p}$ is not a full-contact node there is at least one contribution in the right hand side of the first condition. Inserting the ansatz (46) in the aforementioned conditions, we get a solvable system of equations with $d+1$ coefficients (degrees of freedom) $a_{M}, a_{i}, i=\{1, . ., d\}$ and $d+1$ conditions. At this point the special choice of $c_{p}(\varphi):=\frac{\int_{\tilde{\gamma}_{p, C}} \varphi \phi_{p}}{\int_{\tilde{\gamma}_{p, C}} \phi_{p}}$ as mean value on $\tilde{\gamma}_{p, C}$ for semi-contact nodes becomes important because the choice $c_{p}(\varphi)=\frac{\int_{\gamma_{p, C}} \varphi \phi_{p}}{\int_{\gamma_{p, C}} \phi_{p}}$ as mean value over the whole patch $\gamma_{p, C}$ would lead to a contradiction of the conditions. In the second condition $\mathfrak{s}_{i}$ would be replaced by $\mathfrak{s}$ and the condition $\int_{\mathfrak{s}} \theta_{\mathfrak{s}} \phi_{p_{i}}=0$ for all $\boldsymbol{p}_{i}$ of the side $\mathfrak{s}$ would imply $\sum_{p \in N_{\mathfrak{m}} \backslash N_{\mathfrak{m}}^{\mathrm{fC}}} \int_{\mathfrak{s}} \theta_{\mathfrak{s}} \phi_{p}=0$ such that the first condition could not be fulfilled.

As we assumed that the mesh is made of simplices, $\hat{\sigma}_{1}\left(\boldsymbol{u}_{\mathfrak{m}}\right)$ is constant on $\mathfrak{s}$. Consequently, $c_{p}\left(\theta_{\mathfrak{s}}\right)=0$ implies $c_{p}\left(\hat{\sigma}_{1}\left(\boldsymbol{u}_{\mathfrak{m}}\right) \theta_{\mathfrak{s}}\right)=0$ and it follows from the first condition

$$
\left\|\hat{\sigma}_{1}\left(\boldsymbol{u}_{\mathfrak{m}}\right)\right\|_{\mathfrak{s}}^{2}=\sum_{p \in N_{\mathfrak{m}} \backslash N_{\mathfrak{m}}^{\mathrm{fC}}} \int_{\mathfrak{s}} \hat{\sigma}_{1}\left(\boldsymbol{u}_{\mathfrak{m}}\right) \hat{\sigma}_{1}\left(\boldsymbol{u}_{\mathfrak{m}}\right) \theta_{\mathfrak{s}} \phi_{p}
$$

Putting together (47), (45) with test function $\hat{\sigma}_{1}\left(\boldsymbol{u}_{\mathfrak{m}}\right) \theta_{\mathfrak{s}}$ instead of $\Psi_{\mathfrak{s}}$ and exploiting the conditions $c_{p}\left(\hat{\sigma}_{1}\left(\boldsymbol{u}_{\mathfrak{m}}\right) \theta_{\mathfrak{s}}\right)=0$ for all contact nodes we end up with

$$
\begin{aligned}
\left\|\hat{\sigma}_{1}\left(\boldsymbol{u}_{\mathfrak{m}}\right)\right\|_{\mathfrak{s}}^{2} & =\sum_{p \in N_{\mathfrak{m}} \backslash N_{\mathfrak{m}}^{\text {fC }}} \int_{\mathfrak{s}} \hat{\sigma}_{1}\left(\boldsymbol{u}_{\mathfrak{m}}\right) \hat{\sigma}_{1}\left(\boldsymbol{u}_{\mathfrak{m}}\right) \theta_{\mathfrak{s}} \phi_{p} \\
& =-\left\langle\mathcal{G}_{\mathfrak{m}}, \hat{\sigma}_{1}\left(\boldsymbol{u}_{\mathfrak{m}}\right) \theta_{\mathfrak{s}} \boldsymbol{e}_{1}\right\rangle+\int_{\omega_{\mathfrak{s}}} r_{1}\left(\boldsymbol{u}_{\mathfrak{m}}\right) \hat{\sigma}_{1}\left(\boldsymbol{u}_{\mathfrak{m}}\right) \theta_{\mathfrak{s}} \\
& \leq\left\|\mathcal{G}_{\mathfrak{m}}\right\|_{-1, \omega_{\bar{p}}}\left\|\hat{\sigma}_{1}\left(\boldsymbol{u}_{\mathfrak{m}}\right) \theta_{\mathfrak{s}}\right\|_{1, \omega_{\mathfrak{s}}}+\left\|r_{1}\left(\boldsymbol{u}_{\mathfrak{m}}\right)\right\|_{\omega_{\mathfrak{s}}}\left\|\hat{\sigma}_{1}\left(\boldsymbol{u}_{\mathfrak{m}}\right) \theta_{\mathfrak{s}}\right\|_{\omega_{\mathfrak{s}}} \\
& \lesssim\left\|\mathcal{G}_{\mathfrak{m}}\right\|_{-1, \omega_{\bar{p}}} h_{\mathfrak{s}}^{-\frac{1}{2}}\left\|\hat{\sigma}_{1}\left(\boldsymbol{u}_{\mathfrak{m}}\right)\right\|_{\mathfrak{s}}+h_{\mathfrak{s}}^{\frac{1}{2}}\left\|r_{1}\left(\boldsymbol{u}_{\mathfrak{m}}\right)\right\|_{\omega_{\mathfrak{s}}}\left\|\hat{\sigma}_{1}\left(\boldsymbol{u}_{\mathfrak{m}}\right)\right\|_{\mathfrak{s}}
\end{aligned}
$$


where $h_{\mathfrak{s}}:=\operatorname{diam}(\mathfrak{s})$. In the last line of (48) we used the properties of the bubble functions on the subgrid and the fact that $\tilde{\gamma}_{p, C}$ is a fixed portion of $\gamma_{p, C}$ so that $h_{\mathfrak{s}}=c h_{\mathfrak{s}_{i}}$ for a mesh-independent constant $c$. We divide by $h_{\mathfrak{s}}^{-\frac{1}{2}}\left\|\hat{\sigma}_{1}\left(\boldsymbol{u}_{\mathfrak{m}}\right)\right\|_{\mathfrak{s}}$ leading to

$$
h_{\mathfrak{s}}^{\frac{1}{2}}\left\|\hat{\sigma}_{1}\left(\boldsymbol{u}_{\mathfrak{m}}\right)\right\|_{\mathfrak{s}} \lesssim\left\|\mathcal{G}_{\mathfrak{m}}\right\|_{-1, \omega_{\bar{p}}}+h_{\mathfrak{s}}\left\|\boldsymbol{r}\left(\boldsymbol{u}_{\mathfrak{m}}\right)\right\|_{\omega_{\mathfrak{s}}}
$$

By means of the triangle inequality, the shape-regularity, $h_{\mathfrak{s}} \approx h_{p}$ and the upper bounds (43) and (42) of $\left\|\mathcal{G}_{\mathfrak{m}}\right\|_{-1, \omega_{\bar{p}}}$ and $\left\|\boldsymbol{r}\left(\boldsymbol{u}_{\mathfrak{m}}\right)\right\|_{\omega_{\bar{p}}}$, we get

$$
h_{p}^{\frac{1}{2}}\left\|\hat{\sigma}_{1}\left(\boldsymbol{u}_{\mathfrak{m}}\right)\right\|_{\gamma_{\bar{p}, C}} \lesssim\left\|\boldsymbol{u}-\boldsymbol{u}_{\mathfrak{m}}\right\|_{1, \omega_{\bar{p}}}+\left\|\boldsymbol{\lambda}-\tilde{\boldsymbol{\lambda}}_{\mathfrak{m}}\right\|_{-1, \omega_{\bar{p}}}+h_{\bar{p}}\|\overline{\boldsymbol{f}}-\boldsymbol{f}\|_{\omega_{\bar{p}}}
$$

Thus, we have proven Theorem 2 for $\eta_{5, \bar{p}}$ with $\bar{p} \in N_{\mathfrak{m}}^{C} \backslash N_{\mathfrak{m}}^{\mathrm{fC}}$.

5.2 Lower bound in terms of the contribution $\eta_{6, p}$

We derive a lower bound of the local error in terms of the local contributions of $\eta_{6, p}=$ $\left(s_{p} d_{p}\right)^{\frac{1}{2}}$. If $s_{p}=0$ or $\left(g_{\mathfrak{m}}-u_{\mathfrak{m}, 1}\right)(\boldsymbol{q})=0$ for all neighbouring nodes of $\boldsymbol{p}$ we have $\eta_{6, p}=0$. Therefore, we assume $s_{p}>0$ and $\left(g_{\mathfrak{m}}-u_{\mathfrak{m}, 1}\right)(\boldsymbol{q})>0$ for at least one node on $\gamma_{p, C}$. Let $\hat{\boldsymbol{q}}$ be a node which fulfills $\left(g_{\mathfrak{m}}-u_{\mathfrak{m}, 1}\right)(\hat{\boldsymbol{q}}) \geq\left(g_{\mathfrak{m}}-u_{\mathfrak{m}, 1}\right)(\boldsymbol{q})$ for all neighboring nodes $\boldsymbol{q}$ of $\boldsymbol{p}$. Due to $s_{p}>0$ we have $\left(g_{\mathfrak{m}}-u_{\mathfrak{m}, 1}\right)(\boldsymbol{p})=0$. As we consider boundary meshes of triangles and intervals the discrete functions are piecewise linear. Using Taylor expansion around $\left(g_{\mathfrak{m}}-u_{\mathfrak{m}, 1}\right)(\boldsymbol{p})=0$ leads to

$$
\begin{aligned}
& \left(g_{\mathfrak{m}}-u_{\mathfrak{m}, 1}\right)(\hat{\boldsymbol{q}}) \\
& =\left.\left.\nabla\right|_{\hat{\mathfrak{s}}}\left(g_{\mathfrak{m}}-u_{\mathfrak{m}, 1}\right) \cdot(\hat{\boldsymbol{q}}-\boldsymbol{p}) \lesssim h_{p} \nabla\right|_{\hat{\mathfrak{s}}}\left(g_{\mathfrak{m}}-u_{\mathfrak{m}, 1}\right) \cdot \boldsymbol{\tau}
\end{aligned}
$$

where $\hat{\mathfrak{s}} \subset \gamma_{p, C}$ is a side containing the nodes $\hat{\boldsymbol{q}}$ and $\boldsymbol{p}$ and $\boldsymbol{\tau}$ is the unit tangential vector pointing from $\boldsymbol{p}$ to $\hat{\boldsymbol{q}}$. The corresponding element is denoted with $\hat{\mathfrak{e}}$.

First, we deal with a node $p$ at a convex edge of the boundary. We assumed in Theorem 2 that the node $\boldsymbol{p}$ has at least one neighboring node in the interior. Thus, there exists another element $\mathfrak{e} \subset \omega_{p}$ with a boundary side $\mathfrak{s} \subset \partial \omega_{p} \cap \Gamma$.

We denote the unit vector pointing from $p$ to the interior node along the interior edge of $\mathfrak{e}$ by $\boldsymbol{\tau}_{\text {int }}$. Further, we define an extension from $g_{\mathfrak{m}}$ to a function in $\mathcal{H}_{\mathfrak{m}}$ by $\bar{g}_{\mathfrak{m}}(\boldsymbol{q})=u_{\mathfrak{m}, 1}(\boldsymbol{q})$ $\forall \boldsymbol{q} \in N_{\mathfrak{m}} \backslash N_{\mathfrak{m}}^{C}$. Due to the definition of $\bar{g}_{\mathfrak{m}}$, we have

$$
\left.\nabla\right|_{\mathfrak{e}}\left(\bar{g}_{\mathfrak{m}}-u_{\mathfrak{m}, 1}\right) \cdot \boldsymbol{\tau}_{\text {int }}=0
$$

For the gradients in direction $\tau_{\text {bd }}$ pointing from $\boldsymbol{p}$ to a neighboring boundary node $\boldsymbol{q} \neq \hat{\boldsymbol{q}}$ we have

$$
\left.\nabla\right|_{\mathfrak{e}}\left(\bar{g}_{\mathfrak{m}}-u_{\mathfrak{m}, 1}\right) \cdot \tau_{\mathrm{bd}} \geq 0
$$




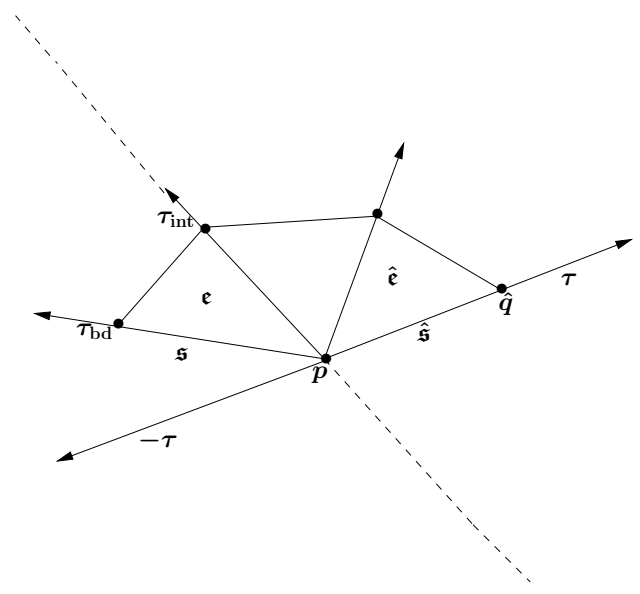

Fig. 2: Construction of the linear combination of $-\tau$ in $2 D$

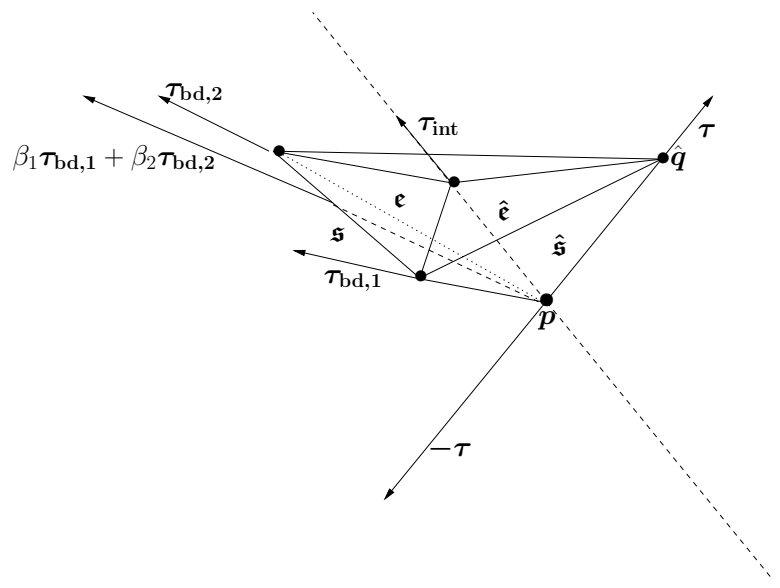

Fig. 3: Construction of the linear combination of $-\tau$ in $3 D$

In the case $d=2$ the line given by $\boldsymbol{p}$ and the vector $\boldsymbol{\tau}_{\text {int }}$ divides the plane into half-planes with $\tau$ on one side and $\tau_{\text {bd }}$ and $-\tau$ on the other side, see Figure 2. Therefore,

$$
-\boldsymbol{\tau}=\alpha \boldsymbol{\tau}_{\text {int }}+\beta \boldsymbol{\tau}_{\text {bd }}
$$

with $\beta>0$ and $\alpha$ arbitrary. If $d=3$ imagine a plane $P_{\tau, \tau_{\text {int }}}$ spanned by $\tau$ and $\tau_{\text {int }}$. The cut of the plane with an opposite side $\mathfrak{s}$ defines a unit vector $\boldsymbol{\tau}_{\mathrm{bd}}:=\beta_{1} \boldsymbol{\tau}_{\mathrm{bd}, 1}+\beta_{2} \boldsymbol{\tau}_{\mathrm{bd}, 2}$ with $\beta_{1}, \beta_{2} \geq 0$ and $\boldsymbol{\tau}_{\mathrm{bd}, 1}, \boldsymbol{\tau}_{\mathrm{bd}, 2}$ are the vectors along the edges of the side $\mathfrak{s}$ starting in $\boldsymbol{p}$, compare 
Figure 3. The line $\tau_{\text {int }}$ divides this plane $P_{\tau, \tau_{\text {int }}}$ such that $-\tau$ and $\tau_{\text {bd }}$ lie in one half-plane and (52) holds with $\beta>0$ and $\alpha$ arbitrary. Putting together (50), (51) and (52), we get

$$
-\left.\nabla\right|_{\mathfrak{e}}\left(\bar{g}_{\mathfrak{m}}-u_{\mathfrak{m}, 1}\right) \cdot \boldsymbol{\tau} \geq 0
$$

and therefore adding (53) to (49) gives

$$
\begin{aligned}
\left(\bar{g}_{\mathfrak{m}}-u_{\mathfrak{m}, 1}\right)(\hat{\boldsymbol{q}}) & =\left.\nabla\right|_{\hat{\mathfrak{s}}}\left(\bar{g}_{\mathfrak{m}}-u_{\mathfrak{m}, 1}\right) \cdot(\hat{\boldsymbol{q}}-\boldsymbol{p}) \\
& \lesssim h_{p}\left(\left.\nabla\right|_{\hat{\mathfrak{e}}}\left(\bar{g}_{\mathfrak{m}}-u_{\mathfrak{m}, 1}\right)-\left.\nabla\right|_{\mathfrak{e}}\left(\bar{g}_{\mathfrak{m}}-u_{\mathfrak{m}, 1}\right)\right) \cdot \boldsymbol{\tau} .
\end{aligned}
$$

For a contact node $\boldsymbol{p}$ at a concave edge, the vector pointing from $\boldsymbol{p}$ in direction $-\boldsymbol{\tau}$ goes through another element $\mathfrak{e}$ belonging to $\omega_{p}$. Due to the construction of $\bar{g}_{\mathfrak{m}}$ such that $\bar{g}_{\mathfrak{m}}(\boldsymbol{q})-$ $u_{\mathfrak{m}, 1}(\boldsymbol{q}) \geq 0$, we have

$$
-\left.\nabla\right|_{\mathfrak{e}}\left(\bar{g}_{\mathfrak{m}}-u_{\mathfrak{m}, 1}\right) \cdot \tau \geq 0
$$

and therefore, we can add this gradient in (49) getting (54) also in this case.

Let $\mathfrak{e}_{0}=\hat{\mathfrak{e}}, \mathfrak{e}_{n}=\mathfrak{e}$ and $\mathfrak{e}_{i}, i=1, . ., n-1$ elements between $\mathfrak{e}_{0}$ and $\mathfrak{e}_{n}$. Common sides are denoted with $\mathfrak{s}_{i}:=\mathfrak{e}_{i-1} \cap \mathfrak{e}_{i}$. Further, we define $\overline{\boldsymbol{v}}_{\mathfrak{m}}$ with $\bar{v}_{\mathfrak{m}, 1}:=\bar{g}_{\mathfrak{m}}-u_{\mathfrak{m}, 1}$ and $\bar{v}_{\mathfrak{m}, i}:=$ $-u_{\mathfrak{m}, i}$ for $i=2, . ., d$. The difference $\left(\left.\nabla\right|_{\hat{\mathfrak{e}}} \bar{v}_{\mathfrak{m}, 1}-\left.\nabla\right|_{\mathfrak{e}} \bar{v}_{\mathfrak{m}, 1}\right) \cdot \tau$ occurring in the right hand side of (54) can be reformulated to

$$
\begin{aligned}
\left(\left.\nabla\right|_{\hat{\mathfrak{e}}} \overline{\boldsymbol{v}}_{\mathfrak{m}, 1}-\left.\nabla\right|_{\mathfrak{e}} \bar{v}_{\mathfrak{m}, 1}\right) \cdot \boldsymbol{\tau} & =\left(\left.\nabla\right|_{\hat{\mathfrak{e}}} \overline{\boldsymbol{v}}_{\mathfrak{m}}-\left.\nabla\right|_{\mathfrak{e}} \overline{\boldsymbol{v}}_{\mathfrak{m}}\right)^{T} \boldsymbol{e}_{1} \cdot \boldsymbol{\tau} \\
& =\left(\left.\nabla\right|_{\hat{\mathfrak{e}}} \overline{\boldsymbol{v}}_{\mathfrak{m}}-\left.\nabla\right|_{\mathfrak{e}} \overline{\boldsymbol{v}}_{\mathfrak{m}}\right) \boldsymbol{\tau} \cdot \boldsymbol{e}_{1} \\
& =\sum_{i=1}^{n}\left(\left.\nabla\right|_{\mathfrak{e}_{i}} \overline{\boldsymbol{v}}_{\mathfrak{m}}-\left.\nabla\right|_{\mathfrak{e}_{i-1}} \overline{\boldsymbol{v}}_{\mathfrak{m}}\right) \boldsymbol{\tau} \cdot \boldsymbol{e}_{1}
\end{aligned}
$$

The unit outward normal to a side $\mathfrak{s}_{i}$ is denoted by $\boldsymbol{n}$ and extended to an orthonormal basis by means of $\boldsymbol{t}_{j}$, for $j=1, . ., d-1$. We can write $\boldsymbol{\tau}=\boldsymbol{\tau} \cdot \boldsymbol{n} \boldsymbol{n}+\sum_{j=1}^{d-1} \boldsymbol{\tau} \cdot \boldsymbol{t}_{j} \boldsymbol{t}_{j}$ and similarly $\boldsymbol{e}_{1}=\boldsymbol{e}_{1} \cdot \boldsymbol{n n}+\sum_{j=1}^{d-1} \boldsymbol{e}_{1} \cdot \boldsymbol{t}_{j} \boldsymbol{t}_{j}$. For each summand in the right hand side of (55), we get

$$
\begin{aligned}
& \left(\left.\nabla\right|_{\mathfrak{e}_{i}} \overline{\boldsymbol{v}}_{\mathfrak{m}}-\left.\nabla\right|_{\mathfrak{e}_{i-1}} \overline{\boldsymbol{v}}_{\mathfrak{m}}\right) \boldsymbol{\tau} \cdot \boldsymbol{e}_{1} \\
= & \boldsymbol{\tau} \cdot \boldsymbol{n}\left(\left.\nabla\right|_{\mathfrak{e}_{i}} \overline{\boldsymbol{v}}_{\mathfrak{m}}-\left.\nabla\right|_{\mathfrak{e}_{i-1}} \overline{\boldsymbol{v}}_{\mathfrak{m}}\right) \boldsymbol{n} \cdot \boldsymbol{e}_{1}+\sum_{j=1}^{d-1} \boldsymbol{\tau} \cdot \boldsymbol{t}_{j} \underbrace{\left(\left.\nabla\right|_{\mathfrak{e}_{i}} \overline{\boldsymbol{v}}_{\mathfrak{m}}-\left.\nabla\right|_{\mathfrak{e}_{i-1}} \overline{\boldsymbol{v}}_{\mathfrak{m}}\right) \boldsymbol{t}_{j}}_{=0} \cdot \boldsymbol{e}_{1} \\
= & \boldsymbol{\tau} \cdot \boldsymbol{n}\left(\left.\nabla\right|_{\mathfrak{e}_{i}} \overline{\boldsymbol{v}}_{\mathfrak{m}}-\left.\nabla\right|_{\mathfrak{e}_{i-1}} \overline{\boldsymbol{v}}_{\mathfrak{m}}\right) \boldsymbol{n} \cdot \boldsymbol{e}_{1} .
\end{aligned}
$$

Exploiting

$$
\begin{aligned}
& \left(\left.\nabla\right|_{\mathfrak{e}_{i}} \overline{\boldsymbol{v}}_{\mathfrak{m}}-\left.\nabla\right|_{\mathfrak{e}_{i-1}} \overline{\boldsymbol{v}}_{\mathfrak{m}}\right) \boldsymbol{n} \cdot \boldsymbol{n} \\
= & \frac{1}{2}\left(\left.\nabla\right|_{\mathfrak{e}_{i}} \overline{\boldsymbol{v}}_{\mathfrak{m}}-\left.\nabla\right|_{\mathfrak{e}_{i-1}} \overline{\boldsymbol{v}}_{\mathfrak{m}}\right) \boldsymbol{n} \cdot \boldsymbol{n}+\frac{1}{2}\left(\left.\nabla\right|_{\mathfrak{e}_{i}} \overline{\boldsymbol{v}}_{\mathfrak{m}}-\left.\nabla\right|_{\mathfrak{e}_{i-1}} \overline{\boldsymbol{v}}_{\mathfrak{m}}\right)^{T} \boldsymbol{n} \cdot \boldsymbol{n} \\
= & \left(\left.\boldsymbol{\epsilon}\left(\overline{\boldsymbol{v}}_{\mathfrak{m}}\right)\right|_{\mathfrak{e}_{i}}-\left.\boldsymbol{\epsilon}\left(\overline{\boldsymbol{v}}_{\mathfrak{m}}\right)\right|_{\mathfrak{e}_{i-1}}\right) \boldsymbol{n} \cdot \boldsymbol{n}
\end{aligned}
$$

and

$$
\begin{aligned}
& \left(\left.\nabla\right|_{\mathfrak{e}_{i}} \overline{\boldsymbol{v}}_{\mathfrak{m}}-\left.\nabla\right|_{\mathfrak{e}_{i-1}} \overline{\boldsymbol{v}}_{\mathfrak{m}}\right) \boldsymbol{n} \cdot \boldsymbol{t}_{j} \\
= & \left(\left.\nabla\right|_{\mathfrak{e}_{i}} \overline{\boldsymbol{v}}_{\mathfrak{m}}-\left.\nabla\right|_{\mathfrak{e}_{i-1}} \overline{\boldsymbol{v}}_{\mathfrak{m}}\right) \boldsymbol{n} \cdot \boldsymbol{t}_{j}+\boldsymbol{n} \cdot\left(\left.\nabla\right|_{\mathfrak{e}_{i}} \overline{\boldsymbol{v}}_{\mathfrak{m}}-\left.\nabla\right|_{\mathfrak{e}_{i-1}} \overline{\boldsymbol{v}}_{\mathfrak{m}}\right) \boldsymbol{t}_{j} \\
= & \left(\left.\nabla\right|_{\mathfrak{e}_{i}} \overline{\boldsymbol{v}}_{\mathfrak{m}}-\left.\nabla\right|_{\mathfrak{e}_{i-1}} \overline{\boldsymbol{v}}_{\mathfrak{m}}\right) \boldsymbol{n} \cdot \boldsymbol{t}_{j}+\left(\left.\nabla\right|_{\mathfrak{e}_{i}} \overline{\boldsymbol{v}}_{\mathfrak{m}}-\left.\nabla\right|_{\mathfrak{e}_{i-1}} \overline{\boldsymbol{v}}_{\mathfrak{m}}\right)^{T} \boldsymbol{n} \cdot \boldsymbol{t}_{j} \\
= & 2\left(\left.\boldsymbol{\epsilon}\left(\overline{\boldsymbol{v}}_{\mathfrak{m}}\right)\right|_{\mathfrak{e}_{i}}-\left.\boldsymbol{\epsilon}\left(\overline{\boldsymbol{v}}_{\mathfrak{m}}\right)\right|_{\mathfrak{e}_{i-1}}\right) \boldsymbol{n} \cdot \boldsymbol{t}_{j},
\end{aligned}
$$


we estimate

$$
\begin{aligned}
& \left(\left.\nabla\right|_{\mathfrak{e}_{i}} \overline{\boldsymbol{v}}_{\mathfrak{m}}-\left.\nabla\right|_{\mathfrak{e}_{i-1}} \overline{\boldsymbol{v}}_{\mathfrak{m}}\right) \boldsymbol{n} \cdot \boldsymbol{e}_{1} \\
= & \boldsymbol{e}_{1} \cdot \boldsymbol{n}\left(\left.\nabla\right|_{\mathfrak{e}_{i}} \overline{\boldsymbol{v}}_{\mathfrak{m}}-\left.\nabla\right|_{\mathfrak{e}_{i-1}} \overline{\boldsymbol{v}}_{\mathfrak{m}}\right) \boldsymbol{n} \cdot \boldsymbol{n}+\sum_{j=1}^{d-1} \boldsymbol{e}_{1} \cdot \boldsymbol{t}_{j}\left(\left.\nabla\right|_{\mathfrak{e}_{i}} \overline{\boldsymbol{v}}_{\mathfrak{m}}-\left.\nabla\right|_{\mathfrak{e}_{i-1}} \overline{\boldsymbol{v}}_{\mathfrak{m}}\right) \boldsymbol{n} \cdot \boldsymbol{t}_{j} \\
\lesssim & \left|\left(\left.\boldsymbol{\epsilon}\left(\overline{\boldsymbol{v}}_{\mathfrak{m}}\right)\right|_{\mathfrak{e}_{i}}-\left.\boldsymbol{\epsilon}\left(\overline{\boldsymbol{v}}_{\mathfrak{m}}\right)\right|_{\mathfrak{e}_{i-1}}\right) \boldsymbol{n}\right| .
\end{aligned}
$$

Putting together (54), (55), (56) and (57) we get

$$
\begin{aligned}
\left(\bar{g}_{\mathfrak{m}}-u_{\mathfrak{m}, 1}\right)(\hat{\boldsymbol{q}}) & \lesssim h_{p} \sum_{i=1}^{n}\left|\left(\left.\nabla\right|_{\mathfrak{e}_{i}} \overline{\boldsymbol{v}}_{\mathfrak{m}}-\left.\nabla\right|_{\mathfrak{e}_{i-1}} \overline{\boldsymbol{v}}_{\mathfrak{m}}\right) \boldsymbol{\tau} \cdot \boldsymbol{e}_{1}\right| \\
& \lesssim h_{p} \sum_{i=1}^{n}\left|\left(\left.\nabla\right|_{\mathfrak{e}_{i}} \overline{\boldsymbol{v}}_{\mathfrak{m}}-\left.\nabla\right|_{\mathfrak{e}_{i-1}} \overline{\boldsymbol{v}}_{\mathfrak{m}}\right) \boldsymbol{n} \cdot \boldsymbol{e}_{1}\right| \\
& \lesssim h_{p} \sum_{i=1}^{n}\left|\left(\left.\boldsymbol{\epsilon}\left(\overline{\boldsymbol{v}}_{\mathfrak{m}}\right)\right|_{\mathfrak{e}_{i}}-\left.\boldsymbol{\epsilon}\left(\overline{\boldsymbol{v}}_{\mathfrak{m}}\right)\right|_{\mathfrak{e}_{i-1}}\right) \boldsymbol{n}\right|
\end{aligned}
$$

In order to bound the jump in the strain in normal direction by means of the jump in the stress in normal direction, we make use of the material law in linear elasticity

$$
\boldsymbol{\sigma}=\lambda \operatorname{tr}(\boldsymbol{\epsilon}) \boldsymbol{i d}+2 \mu \boldsymbol{\epsilon},
$$

compare (2). The inverse relation is given by

$$
\boldsymbol{\epsilon}=\frac{1}{2 \mu} \boldsymbol{\sigma}-\frac{\lambda}{2 \mu(d \lambda+2 \mu)} \operatorname{tr}(\boldsymbol{\sigma}) \boldsymbol{i d},
$$

where $d$ refers to the dimension. Thus,

$$
\begin{aligned}
& \left|\left(\left.\boldsymbol{\epsilon}\left(\overline{\boldsymbol{v}}_{\mathfrak{m}}\right)\right|_{\mathfrak{e}_{i}}-\left.\boldsymbol{\epsilon}\left(\overline{\boldsymbol{v}}_{\mathfrak{m}}\right)\right|_{\mathfrak{e}_{i-1}}\right) \boldsymbol{n}\right| \\
\lesssim & \left|\left(\left.\boldsymbol{\sigma}\left(\overline{\boldsymbol{v}}_{\mathfrak{m}}\right)\right|_{\mathfrak{e}_{i}}-\left.\boldsymbol{\sigma}\left(\overline{\boldsymbol{v}}_{\mathfrak{m}}\right)\right|_{\mathfrak{e}_{i-1}}\right) \boldsymbol{n}\right|+\left|\operatorname{tr} \boldsymbol{\sigma}\left(\overline{\boldsymbol{v}}_{\mathfrak{m}}\right)\right|_{\mathfrak{e}_{i}}-\left.\operatorname{tr} \boldsymbol{\sigma}\left(\overline{\boldsymbol{v}}_{\mathfrak{m}}\right)\right|_{\mathfrak{e}_{i-1}} \mid
\end{aligned}
$$

and it remains to bound $\left|\operatorname{tr} \sigma\left(\overline{\boldsymbol{v}}_{\mathfrak{m}}\right)\right|_{\mathfrak{e}_{i}}-\left.\operatorname{tr} \boldsymbol{\sigma}\left(\overline{\boldsymbol{v}}_{\mathfrak{m}}\right)\right|_{\mathfrak{e}_{i-1}} \mid$ by means of the jumps in the stress in normal direction. The trace is invariant under a basis transformation, thus

$$
\begin{aligned}
& \left.\operatorname{tr} \boldsymbol{\sigma}\left(\overline{\boldsymbol{v}}_{\mathfrak{m}}\right)\right|_{\mathfrak{e}_{i}}-\left.\operatorname{tr} \boldsymbol{\sigma}\left(\overline{\boldsymbol{v}}_{\mathfrak{m}}\right)\right|_{\mathfrak{e}_{i-1}} \\
= & \left(\left.\boldsymbol{\sigma}\left(\overline{\boldsymbol{v}}_{\mathfrak{m}}\right)\right|_{\mathfrak{e}_{i}}-\left.\boldsymbol{\sigma}\left(\overline{\boldsymbol{v}}_{\mathfrak{m}}\right)\right|_{\mathfrak{e}_{i-1}}\right) \boldsymbol{n} \cdot \boldsymbol{n}+\sum_{j=1}^{d-1}\left(\left.\boldsymbol{\sigma}\left(\overline{\boldsymbol{v}}_{\mathfrak{m}}\right)\right|_{\mathfrak{e}_{i}}-\left.\boldsymbol{\sigma}\left(\overline{\boldsymbol{v}}_{\mathfrak{m}}\right)\right|_{\mathfrak{e}_{i-1}}\right) \boldsymbol{t}_{j} \cdot \boldsymbol{t}_{j}
\end{aligned}
$$

and it remains to bound $\left(\left.\boldsymbol{\sigma}\left(\overline{\boldsymbol{v}}_{\mathfrak{m}}\right)\right|_{\mathfrak{e}_{i}}-\left.\boldsymbol{\sigma}\left(\overline{\boldsymbol{v}}_{\mathfrak{m}}\right)\right|_{\mathfrak{e}_{i-1}}\right) \boldsymbol{t}_{j} \boldsymbol{t}_{j}$. To this end, we make use of

$$
\begin{aligned}
& \left(\left.\boldsymbol{\epsilon}\left(\overline{\boldsymbol{v}}_{\mathfrak{m}}\right)\right|_{\mathfrak{e}_{i}}-\left.\boldsymbol{\epsilon}\left(\overline{\boldsymbol{v}}_{\mathfrak{m}}\right)\right|_{\mathfrak{e}_{i-1}}\right) \boldsymbol{t}_{j} \cdot \boldsymbol{t}_{j} \\
= & \frac{1}{2} \underbrace{\left(\left.\nabla \overline{\boldsymbol{v}}\right|_{\mathfrak{e}_{i}}-\left.\nabla \overline{\boldsymbol{v}}\right|_{\mathfrak{e}_{i-1}}\right) \boldsymbol{t}_{j}}_{=0} \cdot \boldsymbol{t}_{j}+\frac{1}{2} \boldsymbol{t}_{j} \underbrace{\left(\left.\nabla \overline{\boldsymbol{v}}\right|_{\mathfrak{e}_{i}}-\left.\nabla \overline{\boldsymbol{v}}\right|_{\mathfrak{e}_{i-1}}\right) \boldsymbol{t}_{j}}_{=0}=0
\end{aligned}
$$

and

$$
\begin{aligned}
& \left.\operatorname{tr} \boldsymbol{\epsilon}\left(\overline{\boldsymbol{v}}_{\mathfrak{m}}\right)\right|_{\mathfrak{e}_{i}}-\left.\operatorname{tr} \boldsymbol{\epsilon}\left(\overline{\boldsymbol{v}}_{\mathfrak{m}}\right)\right|_{\mathfrak{e}_{i-1}} \\
= & \left(\left.\boldsymbol{\epsilon}\left(\overline{\boldsymbol{v}}_{\mathfrak{m}}\right)\right|_{\mathfrak{e}_{i}}-\left.\boldsymbol{\epsilon}\left(\overline{\boldsymbol{v}}_{\mathfrak{m}}\right)\right|_{\mathfrak{e}_{i-1}}\right) \boldsymbol{n} \cdot \boldsymbol{n}+\sum_{j=1}^{d-1}\left(\left.\boldsymbol{\epsilon}\left(\overline{\boldsymbol{v}}_{\mathfrak{m}}\right)\right|_{\mathfrak{e}_{i}}-\left.\boldsymbol{\epsilon}\left(\overline{\boldsymbol{v}}_{\mathfrak{m}}\right)\right|_{\mathfrak{e}_{i-1}}\right) \boldsymbol{t}_{j} \cdot \boldsymbol{t}_{j} \\
= & \left(\left.\boldsymbol{\epsilon}\left(\overline{\boldsymbol{v}}_{\mathfrak{m}}\right)\right|_{\mathfrak{e}_{i}}-\left.\boldsymbol{\epsilon}\left(\overline{\boldsymbol{v}}_{\mathfrak{m}}\right)\right|_{\mathfrak{e}_{i-1}}\right) \boldsymbol{n} \cdot \boldsymbol{n}
\end{aligned}
$$


where we exploited (61) and again the fact that the trace is invariant under a basis transformation. Furthermore, we use the identity

$$
\begin{aligned}
& \left(\left.\boldsymbol{\sigma}\left(\overline{\boldsymbol{v}}_{\mathfrak{m}}\right)\right|_{\mathfrak{e}_{i}}-\left.\boldsymbol{\sigma}\left(\overline{\boldsymbol{v}}_{\mathfrak{m}}\right)\right|_{\mathfrak{e}_{i-1}}\right) \boldsymbol{n} \cdot \boldsymbol{n} \\
= & \lambda\left(\left.\operatorname{tr} \boldsymbol{\epsilon}\left(\overline{\boldsymbol{v}}_{\mathfrak{m}}\right)\right|_{\mathfrak{e}_{i}}-\left.\operatorname{tr} \boldsymbol{\epsilon}\left(\overline{\boldsymbol{v}}_{\mathfrak{m}}\right)\right|_{\mathfrak{e}_{i-1}}\right)+2 \mu\left(\left.\boldsymbol{\epsilon}\left(\overline{\boldsymbol{v}}_{\mathfrak{m}}\right)\right|_{\mathfrak{e}_{i}}-\left.\boldsymbol{\epsilon}\left(\overline{\boldsymbol{v}}_{\mathfrak{m}}\right)\right|_{\mathfrak{e}_{i-1}}\right) \boldsymbol{n} \cdot \boldsymbol{n} \\
= & (\lambda+2 \mu)\left(\left.\operatorname{tr} \boldsymbol{\epsilon}\left(\overline{\boldsymbol{v}}_{\mathfrak{m}}\right)\right|_{\mathfrak{e}_{i}}-\left.\operatorname{tr} \boldsymbol{\epsilon}\left(\overline{\boldsymbol{v}}_{\mathfrak{m}}\right)\right|_{\mathfrak{e}_{i-1}}\right)
\end{aligned}
$$

which follows from the material law and from (62). Thus,

$$
\begin{aligned}
& \left(\left.\boldsymbol{\sigma}\left(\overline{\boldsymbol{v}}_{\mathfrak{m}}\right)\right|_{\mathfrak{e}_{i}}-\left.\boldsymbol{\sigma}\left(\overline{\boldsymbol{v}}_{\mathfrak{m}}\right)\right|_{\mathfrak{e}_{i-1}}\right) \boldsymbol{t}_{j} \cdot \boldsymbol{t}_{j} \\
= & \lambda\left(\left.\operatorname{tr} \boldsymbol{\epsilon}\left(\overline{\boldsymbol{v}}_{\mathfrak{m}}\right)\right|_{\mathfrak{e}_{i}}-\left.\operatorname{tr} \boldsymbol{\epsilon}\left(\overline{\boldsymbol{v}}_{\mathfrak{m}}\right)\right|_{\mathfrak{e}_{i-1}}\right)+2 \mu \underbrace{\left(\left.\boldsymbol{\epsilon}\left(\overline{\boldsymbol{v}}_{\mathfrak{m}}\right)\right|_{\mathfrak{e}_{i}}-\left.\boldsymbol{\epsilon}\left(\overline{\boldsymbol{v}}_{\mathfrak{m}}\right)\right|_{\mathfrak{e}_{i-1}}\right) \boldsymbol{t}_{j} \cdot \boldsymbol{t}_{j}}_{=0} \\
= & \frac{\lambda}{\lambda+2 \mu}\left(\left.\boldsymbol{\sigma}\left(\overline{\boldsymbol{v}}_{\mathfrak{m}}\right)\right|_{\mathfrak{e}_{i}}-\left.\boldsymbol{\sigma}\left(\overline{\boldsymbol{v}}_{\mathfrak{m}}\right)\right|_{\mathfrak{e}_{i-1}}\right) \boldsymbol{n} \cdot \boldsymbol{n}
\end{aligned}
$$

where we exploited the material law and (63). Combining (64), (60) and (59) we get the desired bound of the jump in the strain in normal direction by means of the jump in the stress in normal direction

$$
\left|\left(\left.\boldsymbol{\epsilon}\left(\overline{\boldsymbol{v}}_{\mathfrak{m}}\right)\right|_{\mathfrak{e}_{i}}-\left.\boldsymbol{\epsilon}\left(\overline{\boldsymbol{v}}_{\mathfrak{m}}\right)\right|_{\mathfrak{e}_{i-1}}\right) \boldsymbol{n}\right| \lesssim\left|\left(\left.\boldsymbol{\sigma}\left(\overline{\boldsymbol{v}}_{\mathfrak{m}}\right)\right|_{\mathfrak{e}_{i}}-\left.\boldsymbol{\sigma}\left(\overline{\boldsymbol{v}}_{\mathfrak{m}}\right)\right|_{\mathfrak{e}_{i-1}}\right) \boldsymbol{n}\right| .
$$

Finally, (58) and (65) give rise to

$$
\begin{aligned}
\left(\bar{g}_{\mathfrak{m}}-u_{\mathfrak{m}, 1}\right)(\hat{\boldsymbol{q}}) & \lesssim h_{p} \sum_{i=1}^{n}\left|\left(\left.\boldsymbol{\sigma}\left(\overline{\boldsymbol{v}}_{\mathfrak{m}}\right)\right|_{\mathfrak{e}_{i}}-\left.\boldsymbol{\sigma}\left(\overline{\boldsymbol{v}}_{\mathfrak{m}}\right)\right|_{\mathfrak{e}_{i-1}}\right) \boldsymbol{n}\right| \\
& \lesssim h_{p} \sum_{i=1}^{n} h_{p}^{\frac{-(d-1)}{2}}\left\|\boldsymbol{J}^{I}\left(\overline{\boldsymbol{v}}_{\mathfrak{m}}\right)\right\|_{\mathfrak{s}_{i}} \\
& \lesssim h_{p}^{\frac{-(d-3)}{2}}\left\|\boldsymbol{J}^{I}\left(\overline{\boldsymbol{v}}_{\mathfrak{m}}\right)\right\|_{\gamma_{p, I}}
\end{aligned}
$$

As $\left(g_{\mathfrak{m}}-u_{\mathfrak{m}, 1}\right)(\boldsymbol{q}) \leq\left(g_{\mathfrak{m}}-u_{\mathfrak{m}, 1}\right)(\hat{\boldsymbol{q}})$ for all $\boldsymbol{q} \in \gamma_{p, C}$, we conclude

$$
\begin{aligned}
d_{p} & =\int_{\tilde{\gamma}_{p, C}}\left(g_{\mathfrak{m}}-u_{\mathfrak{m}, 1}\right) \phi_{p} \lesssim h_{p}^{d-1} h_{p}^{\frac{-d+2}{2}}\left(h_{p}^{\frac{1}{2}}\left\|\boldsymbol{J}^{I}\left(\overline{\boldsymbol{v}}_{\mathfrak{m}}\right)\right\|_{\gamma_{p, I}}\right) \\
& \lesssim h_{p}^{\frac{d}{2}}\left(h_{p}^{\frac{1}{2}}\left\|\boldsymbol{J}^{I}\left(\begin{array}{c}
\bar{g}_{\mathfrak{m}} \\
0
\end{array}\right)\right\|_{\gamma_{p, I}}+h_{p}^{\frac{1}{2}}\left\|\boldsymbol{J}^{I}\left(\boldsymbol{u}_{\mathfrak{m}}\right)\right\|_{\gamma_{p, I}}\right) .
\end{aligned}
$$


For the upper bound of $s_{p} d_{p}$, we use Hölder's inequality and scaling arguments such that

$$
\begin{aligned}
& s_{p} d_{p} \\
& =\frac{\int_{\gamma_{p, I}} J_{1}^{I}\left(\boldsymbol{u}_{\mathfrak{m}}\right) \phi_{p}+\int_{\omega_{p}} r_{1}\left(\boldsymbol{u}_{\mathfrak{m}}\right) \phi_{p}+\int_{\gamma_{p, N}} J_{1}^{N}\left(\boldsymbol{u}_{\mathfrak{m}}\right) \phi_{p}-\int_{\gamma_{p, C}} \hat{\sigma}_{1}\left(\boldsymbol{u}_{\mathfrak{m}}\right) \phi_{p}}{\int_{\gamma_{p}, C} \phi_{p}} \\
& \cdot \int_{\tilde{\gamma}_{p, C}}\left(g_{\mathfrak{m}}-u_{\mathfrak{m}, 1}\right) \phi_{p} \\
& \lesssim(\left\|J_{1}^{I}\left(\boldsymbol{u}_{\mathfrak{m}}\right)\right\|_{\gamma_{p, I}} \underbrace{\left\|\phi_{p}\right\|_{\gamma_{p, I}}}_{\approx h_{p}^{\frac{d-1}{2}}}+\left\|r_{1}\left(\boldsymbol{u}_{\mathfrak{m}}\right)\right\|_{\omega_{p}} \underbrace{\left\|\phi_{p}\right\|_{\omega_{p}}}_{\approx h_{p}^{\frac{d}{2}}} \\
& +\left\|\hat{\sigma}_{1}\left(\boldsymbol{u}_{\mathfrak{m}}\right)\right\|_{\gamma_{p, C}} \underbrace{\left\|\phi_{p}\right\|_{\gamma_{p, C}}}_{\approx h_{p}^{\frac{d-1}{2}}}) \cdot h_{p}^{-d+1} \int_{\gamma_{p, C}}\left(g_{\mathfrak{m}}-u_{\mathfrak{m}, 1}\right) \phi_{p} \\
& \lesssim\left(h_{p}^{\frac{1}{2}}\left\|J_{1}^{I}\left(\boldsymbol{u}_{\mathfrak{m}}\right)\right\|_{\gamma_{p, I}}+h_{p}\left\|r_{1}\left(\boldsymbol{u}_{\mathfrak{m}}\right)\right\|_{\omega_{p}}+h_{p}^{\frac{1}{2}}\left\|\hat{\sigma}_{1}\left(\boldsymbol{u}_{\mathfrak{m}}\right)\right\|_{\gamma_{p, C}}\right) \\
& \cdot h_{p}^{-\frac{d}{2}} \cdot \int_{\gamma_{p, C}}\left(g_{\mathfrak{m}}-u_{\mathfrak{m}, 1}\right) \phi_{p} \\
& \lesssim\left(\left\|\boldsymbol{u}_{\mathfrak{m}}-\boldsymbol{u}\right\|_{1, \omega_{p}}+\left\|\tilde{\boldsymbol{\lambda}}_{\mathfrak{m}}-\boldsymbol{\lambda}\right\|_{-1, \omega_{p}}+h_{p}\|\overline{\boldsymbol{f}}-\boldsymbol{f}\|_{\omega_{p}}\right) \\
& \cdot\left(h_{p}^{\frac{1}{2}}\left\|\boldsymbol{J}^{I}\left(\begin{array}{c}
\bar{g}_{\mathfrak{m}} \\
0
\end{array}\right)\right\|_{\gamma_{p, I}}+h_{p}^{\frac{1}{2}}\left\|\boldsymbol{J}^{I}\left(\boldsymbol{u}_{\mathfrak{m}}\right)\right\|_{\gamma_{p, I}}\right) \\
& \lesssim\left(\left\|\boldsymbol{u}_{\mathfrak{m}}-\boldsymbol{u}\right\|_{1, \omega_{p}}+\left\|\tilde{\boldsymbol{\lambda}}_{\mathfrak{m}}-\boldsymbol{\lambda}\right\|_{-1, \omega_{p}}+h_{p}\|\overline{\boldsymbol{f}}-\boldsymbol{f}\|_{\omega_{p}}+h_{p}^{\frac{1}{2}}\left\|\boldsymbol{J}^{I}\left(\begin{array}{c}
\bar{g}_{\mathfrak{m}} \\
0
\end{array}\right)\right\|_{\gamma_{p, I}}\right)^{2}
\end{aligned}
$$

where we exploited that the actual contact boundary is a strict subset of the potential contact boundary. Thus, we have proven Theorem 2.

We note that $\boldsymbol{J}^{I}\left(\begin{array}{c}\bar{g}_{\mathfrak{m}} \\ 0\end{array}\right)$ is the counterpart to the jumps in the obstacle function occurring in the local lower bounds in the case of obstacle problems, see [25, 26, 31].

\section{Numerical results}

The implementation of the a posteriori error estimator has been carried out in the framework of the finite element toolbox UG [4] and the obstacle toolbox OBSLIB++, see [23]. In this section we compute the numerical solutions of different contact problems in $2 D$ and $3 D$. The variational inequalities are solved with a non-smooth multigrid method, see [21,23] and the meshes are generated in an adaptive refinement process using red-green-refinement on the basis of our new a posteriori error estimator.

We examine the structure of the refined grids and the rate of convergence of the estimator. In Section 6.1 we consider examples for which the contact stresses are given analytically. 
Thus, we can give the relative error. In Section 6.2 we analyze our residual-type estimator for contact in more detail, i.e., we analyze the relevance of the different error estimator contributions $\eta_{i}$ and the distribution of their local contributions $\eta_{i, p}$.

In all our experiments the force density $f$ and the Neumann values $\boldsymbol{\pi}$ are zero. Although the efficiency and the reliability of the a posteriori error estimator are proven for meshes of simplices, we see in the following examples that the estimator performs very well also for different kinds of meshes.

\subsection{Examples with analytically given distribution of contact stresses}

In the first example a two-dimensional wedge indents a linear elastic half space, see [18, Chapter 5.2]. The semi-angle $\alpha$ of the wedge has to be close to $90^{\circ}$, see Figure 4(a), such that the theory of linear elasticity is valid. The half of the width of the contact strip is

$$
b=\frac{P\left(1-\nu^{2}\right)}{E \cot \alpha}
$$

where $P$ is the load applied to the rigid body and $E$ and $\nu$ are the Young's modulus and the Poisson ratio. The distribution of the contact stresses is given by

$$
\hat{\sigma}_{\nu}(\boldsymbol{x})=\frac{E \cot \alpha}{\left(1-\nu^{2}\right) 2 \pi} \ln \left(\frac{b+\left(b^{2}-x_{1}^{2}\right)^{\frac{1}{2}}}{b-\left(b^{2}-x_{1}^{2}\right)^{\frac{1}{2}}}\right)
$$

where $\nu=e_{2}$ in Figure 4(a). The value of (68) is infinite at the apex of the wedge.

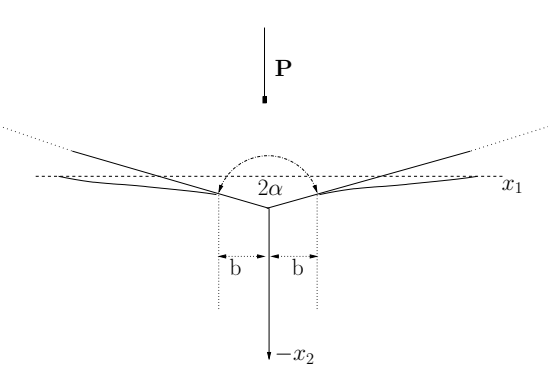

(a) Rigid wedge

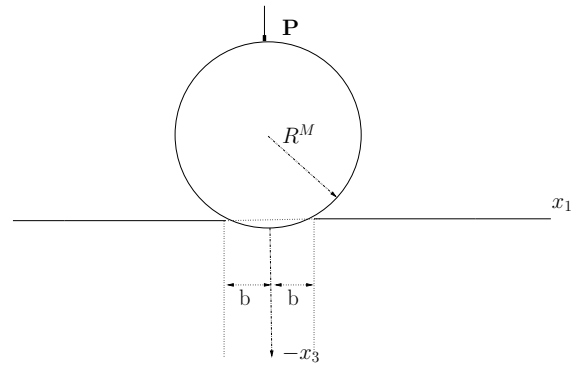

(b) Cross-section of Hertzian contact

Fig. 4

The second example is a special case of the Hertzian contact problem. Originally, the Hertzian contact problem [13] describes the contact between two linear elastic spherical bodies of dimension $d=3$ with radii $R^{S}, R^{M}$ and elastic moduli $E^{S}, E^{M}$ and $\nu^{S}, \nu^{M}$. Here, we consider the extreme case, the contact of a linear elastic cube $\left(R^{S}=\infty\right)$ and a rigid ball $\left(E^{M}=\infty\right)$, see Figure 4(b) for a schematic view of a cross-section. In Figure 4(b) 
$\boldsymbol{\nu}=\boldsymbol{e}_{3}$, so that $\boldsymbol{x}_{T}=\boldsymbol{x}-x_{3} \boldsymbol{e}_{3}$. If the rigid ball is pressed against the linear elastic cube by a load $P$, the radius of the actual contact zone is

$$
b=\left(\frac{3 P R^{M}\left(1-\left(\nu^{S}\right)^{2}\right)}{4 E^{S}}\right)^{\frac{1}{3}}
$$

and the pressure distribution is

$$
\hat{\sigma}_{\nu}(\boldsymbol{x})=\frac{3 P}{2 \pi b^{3}}\left(b^{2}-\left|\boldsymbol{x}_{T}\right|^{2}\right)^{\frac{1}{2}} .
$$

For more details about the Hertzian contact problem, we refer to [18, Chapter 3.4].

As already explained in Section 3.1 the contact force density $\boldsymbol{\lambda}$ is related to the contact stresses $\langle\boldsymbol{\lambda}, \boldsymbol{\varphi}\rangle_{-1,1}=-\left\langle\hat{\sigma}_{\nu}(\boldsymbol{u}), \varphi_{\nu}\right\rangle_{-\frac{1}{2}, \frac{1}{2}, \Gamma_{C}}$ by means of Green's formula, whereas such a relation does not exist in the discrete setting. In order to get a numerical approximation $s_{\mathfrak{m}}$ of $\hat{\sigma}_{\nu}$ we make use of the nodal values of the discrete contact force density obtained by the lumped $L^{2}$-scalar product and define $s_{\mathfrak{m}}:=\sum_{p \in N_{\mathfrak{m}}^{C}} s_{p} \phi_{p}$, such that

$$
\left\langle s_{\mathfrak{m}}, \varphi_{\mathfrak{m}, \nu}\right\rangle_{\Gamma_{C}}=\int_{\Gamma_{C}}\left(\sum_{p \in N_{\mathfrak{m}}^{C}} s_{p} \phi_{p}\right) \varphi_{\mathfrak{m}, \nu}
$$

We call

$$
\frac{\left\|s_{\mathfrak{m}}(\boldsymbol{x})-\hat{\sigma}_{\nu}(\boldsymbol{x})\right\|_{\Gamma_{C}}}{\left\|\hat{\sigma}_{\nu}(\boldsymbol{x})\right\|_{\Gamma_{C}}}
$$

the relative error of the contact stresses in the $L^{2}$-norm.

In order to model the $2 D$ example by a one-body contact problem we consider a unit quadrilateral with the edges $(0,0),(0,1),(1,0),(1,1)$ which is moved towards the obstacle by means of Dirichlet values. We apply Dirichlet boundary values -0.01 in $x_{2}$-direction and 0.0 in $x_{1}$-direction at the top of the quadrilateral. The potential contact boundary is the lower edge of the quadrilateral and the direction of constraints is $\boldsymbol{\nu}=-\boldsymbol{e}_{2}$. The gap function is given by $g\left(x_{1}\right)=0.2 \cdot\left(x_{1}-0.5\right)$ if $x_{1} \geq 0.5$ and $g\left(x_{1}\right)=-0.2 \cdot\left(x_{1}-0.5\right)$ otherwise. The material is assumed to be linear elastic with the Poisson ratio $\nu=0.25$ and the Young's modulus $E=3 \cdot 10^{5} \frac{\mathrm{kN}}{\mathrm{mm}^{2}}$.

The cotangens of the angle $\alpha$ of the rigid wedge and the load applied to the rigid body, compare Figure 4(a) enter in the formulas for the exact computation of the radius of the contact zone and the contact stresses. We note that the load $P$ applied to the rigid body coincides with the integral of the contact stresses over the contact boundary $\int_{\Gamma_{C}} \hat{\sigma}_{\nu} d x_{1}$. Thus, we solve the contact problem on a very fine grid in a preprocess to get a good approximation of the total contact forces. In this way we get the value 872.19. The cotangens of the angle of the wedge is 0.2 . Putting these values in formula (67) we get the radius $b=0.01362796875$ of the contact zone.

If the starting grid consists of uniform quadrilaterals (256 elements) we call the experiment wedge example with quadrilaterals and if the starting grid is made of uniform triangles (512 elements) we refer to it by wedge example with triangles. The adaptive refinement process has been carried out 14 times. The number of degrees of freedom is 510,588 in the wedge example with quadrilaterals and 512,850 in the wedge example with triangles.

Figure 5 shows the adaptively refined grid around the corner caused by the tip of the wedge. In order to visualize the relation between the different zooms in Figure 5 we put 
yellow colored dots below the figures marking the points where $x_{1}=0.4, x_{1}=0.5$ and $x_{1}=0.6$ and green colored dots are set between the yellow colored dots in distances of 0.02 . The refined grid is symmetric and the smallest elements can be found mainly in the area where the tip of the wedge indents the elastic quadrilateral. At this point the exact normal stresses would be infinite. However, in small areas around the free boundary zone the elements have been refined very often, too, as can be seen in Figure 5(d) where we zoomed in the right area of the contact corner. The adaptively refined grid based on a starting grid of triangles is less symmetric, see Figure 6(a). This is due to the alignment of the triangles on the starting grid. However, the solution (Figure 6(b)) is symmetric.

The rate of convergence of the error estimator is given by the so-called experimental order of convergence

$$
\operatorname{eoc}:=\frac{\ln \left(\eta^{k}\right)-\ln \left(\eta^{k+1}\right)}{\ln \left(\# N_{\mathrm{m}, k+1}\right)-\ln \left(\# N_{\mathrm{m}, k}\right)}
$$

where $\eta^{k}, \eta^{k+1}$ are the error estimators of two successive refinement steps with the sets of node $N_{\mathfrak{m}_{k}}, N_{\mathfrak{m}_{k+1}}$. We here take the last two steps of refinement. We note that for first order elements the rate of convergence of the error is optimal if it is $\frac{1}{d}$.

The convergence of the error estimator on adaptively and uniformly refined grids is shown in Figure 7. The experimental order of convergence is about 0.502 for the adaptively refined grid and 0.436 and 0.399 , respectively, for the uniformly refined grid.

In Figure 8 the exact normal stresses $\hat{\sigma}_{\nu}(\boldsymbol{p})$ (blue dots), computed by means of formula (68), are compared to the corresponding numerically computed quantities $s_{p}$ (red dots) on adaptively refined meshes. The values coincide very well.

The $L^{2}$-norm of the relative error between $s_{\mathfrak{m}}(\boldsymbol{x})$ and $\hat{\sigma}_{\nu}(\boldsymbol{x})$ as defined in (71) is given by the red lines in Figure 9 plotted against the number of actual contact nodes $N_{\mathfrak{m}}^{a C}$. The blue lines refer to the same relative error but $s_{\mathfrak{m}}$ is computed on a mesh where the adaptive refinement is determined by the standard residual error estimator of linear elliptic problems. For both methods of adaptive refinement Figure 10 shows the relative error between $b$ and the numerically computed radius of the contact zone plotted against the number of actual contact nodes. In order to visualize that the adaptive refinement process by means of the standard residual error estimator requires much more nodes in order to obtain the same accuracy as by means of our residual-type estimator $\eta$ defined in (22) for contact we have chosen logarithmic scales in Figures 9 and 10.

The $3 D$ example is modeled by a linear elastic unit cube which is pushed against the obstacle by means of Dirichlet values. We apply Dirichlet values -0.005 in $x_{3}$-direction and 0.0 in $x_{1}$ - and $x_{2}$-direction at the top of the cube. The potential contact boundary is the bottom of the cube and the direction of constraints is $\nu=-e_{3}$. The gap function describing the distance between the elastic cube and the rigid ball with radius $R^{M}$ and midpoint $\boldsymbol{m}$ is given by $g=\sqrt{\left(R^{M}\right)^{2}-\left(x_{1}-m_{1}\right)^{2}-\left(x_{2}-m_{2}\right)^{2}}+m_{3}$. The elastic moduli are chosen as in the $2 D$ examples.

We consider two experiments with different starting grids. In the first experiment called Hertzian contact with hexahedra the starting grid is a uniformly refined mesh of hexahedra (64 elements) and in the second experiment called Hertzian contact with tetrahedra the starting grid is a uniformly refined mesh of tetrahedra (384 elements). The final grids have been 10 times adaptively refined. The number of degrees of freedom is 4,871,271 in the experiment Hertzian contact with hexahedra and 6,044,778 in the experiment Hertzian contact with tetrahedra, respectively. 


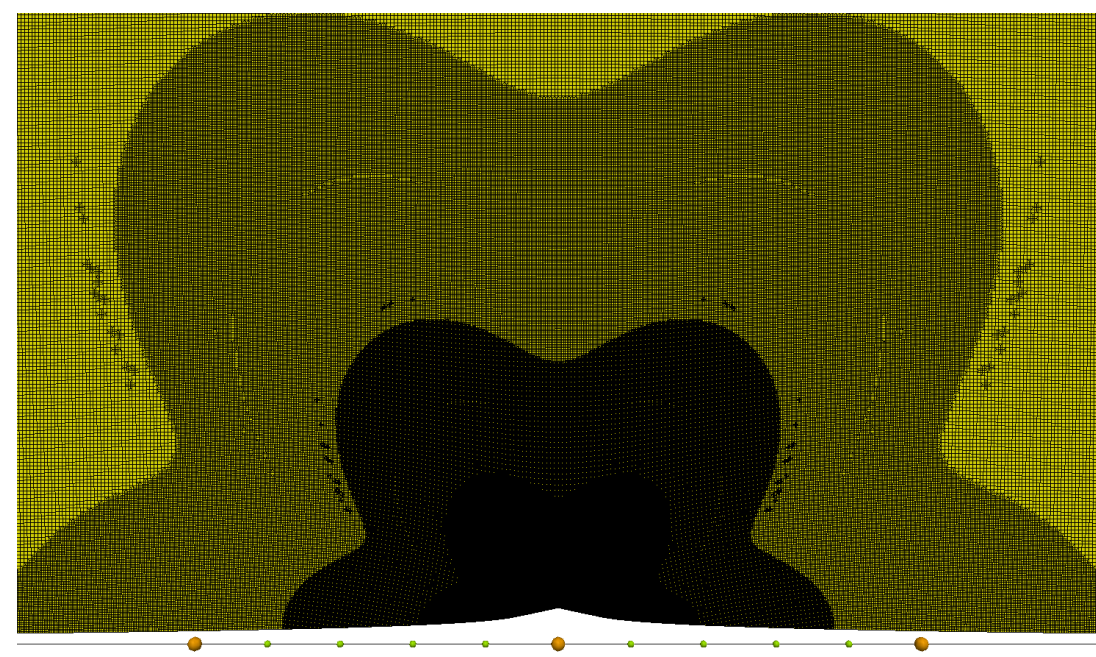

(a) Zoom in contact area of final grid

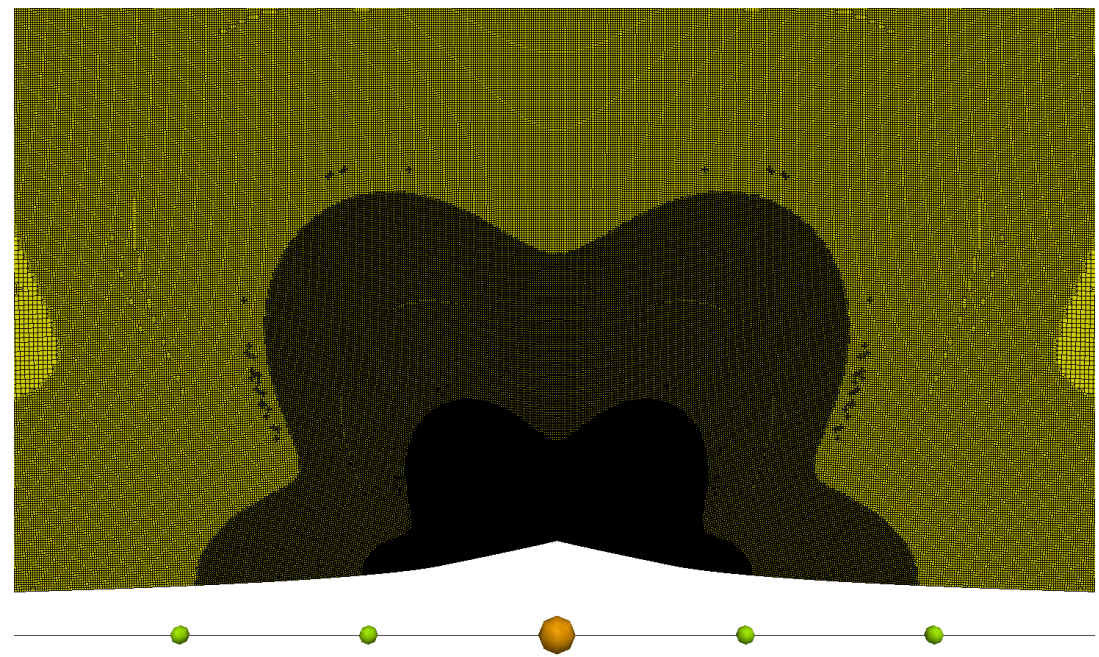

(b) Zoom in contact area of final grid

Fig. 5: Wedge example with quadrilaterals: a)-c). Mesh grading zoomed in the corner caused by the tip of the wedge; d). Mesh grading around the free boundary zone, zoomed in the right area of the corner caused by the tip of the wedge

The value of the load $P$ is computed in a preprocess on a very fine grid and amounts to 88.28. The radius of the rigid ball representing the obstacle is $R^{M}=0.75$. Thus, the radius computed by (69) of the contact zone is 0.615142 .

In Figure 11 we show a zoom of the actual contact boundary of the adaptively refined grids of both examples: Hertzian contact with hexahedra and Hertzian contact with tetrahe$d r a$. The free boundary is more often refined as the area around full-contact nodes. For both experiments, Hertzian contact with hexahedra and Hertzian contact with tetrahedra, the 

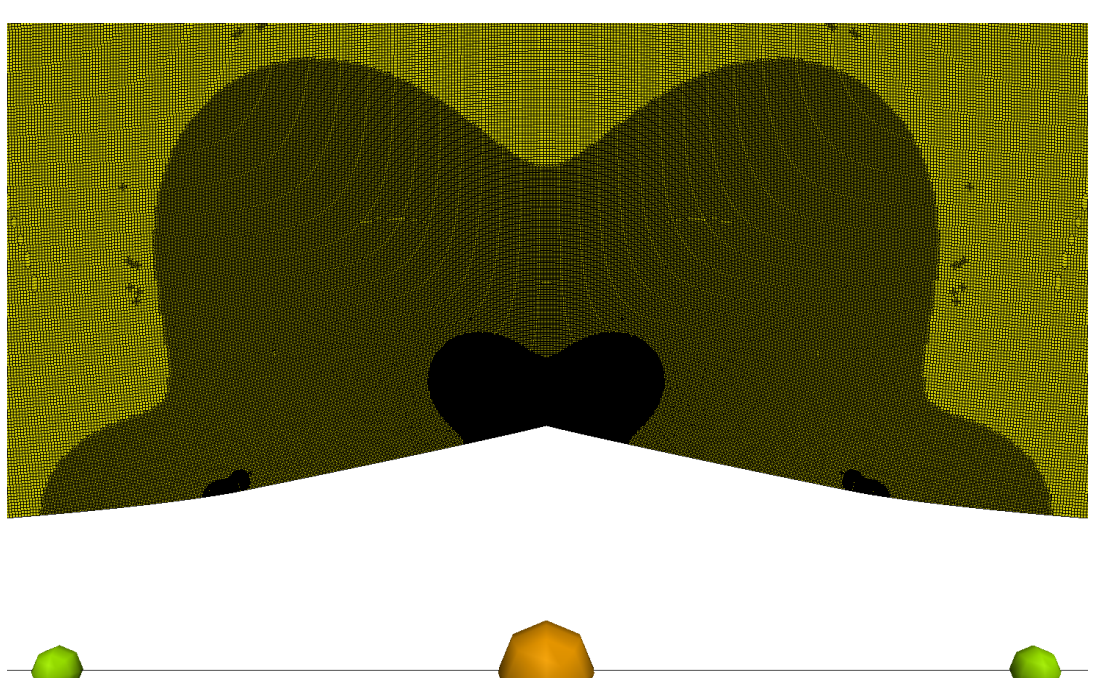

(c) Zoom in contact area of final grid

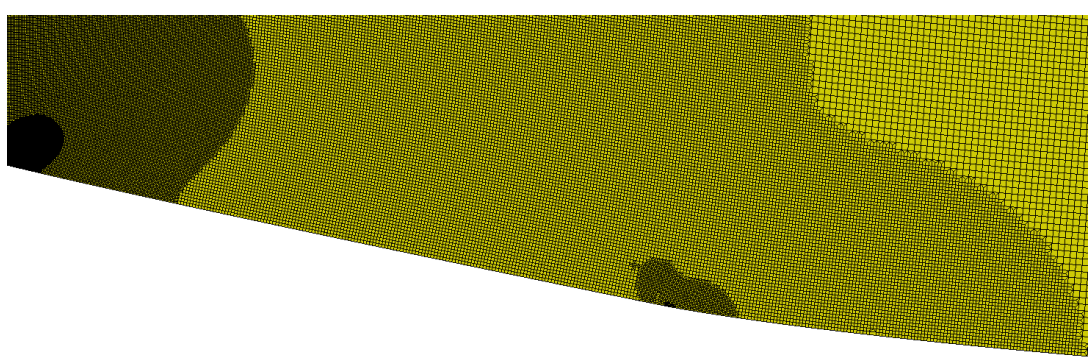

(d) Zoom in contact area of final grid

Fig. 5: Wedge example with quadrilaterals: a)-c). Mesh grading zoomed in the corner caused by the tip of the wedge; d). Mesh grading around the free boundary zone, zoomed in the right area of the corner caused by the tip of the wedge

rates of convergence of our new residual estimator for contact have been plotted in Figure 12 for the adaptively refined grids as well as for the uniformly refined grids.

The $L^{2}$-norm of the relative error (71) between $s_{\mathfrak{m}}(\boldsymbol{x})$ and $\hat{\sigma}_{\nu}(\boldsymbol{x})$ plotted against the number of actual contact nodes is given by the red lines in Figure 13 for both examples. The blue lines in Figure 13 represent the same quantity but $s_{\mathfrak{m}}(\boldsymbol{x})$ is computed on a grid which is adaptively refined by means of the standard residual error estimators. We have chosen 


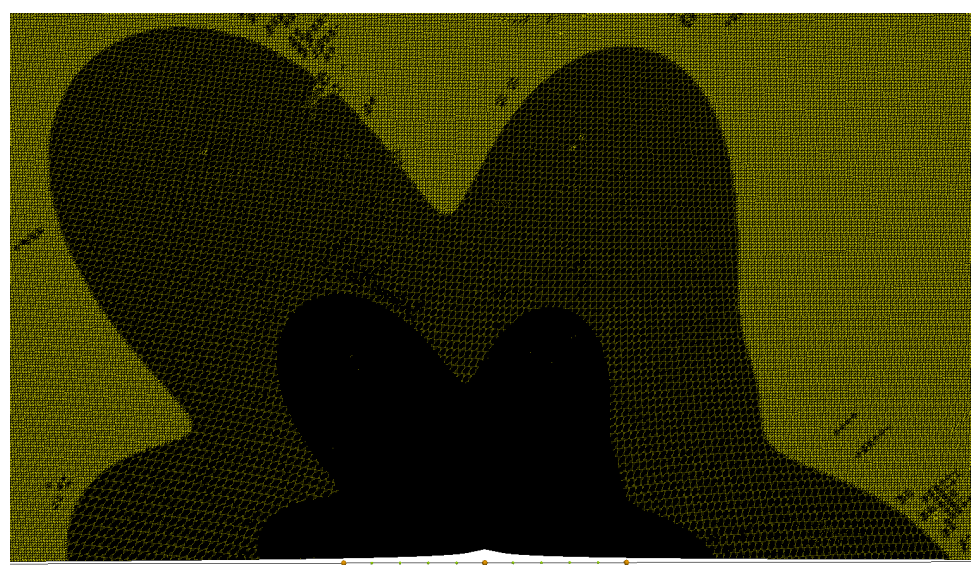

(a) Zoom in contact area of final grid

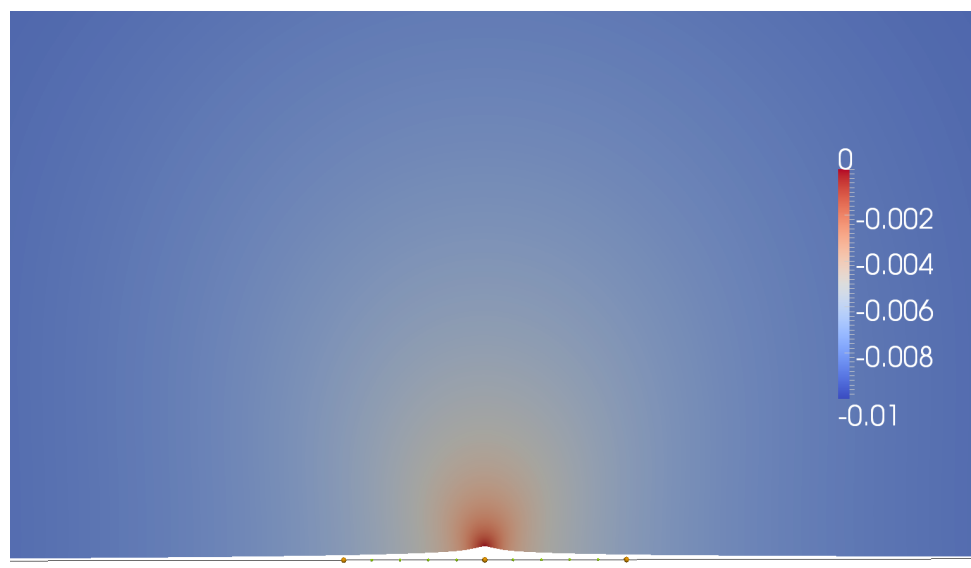

(b) Component $u_{2}$ of the solution

Fig. 6: Wedge example with triangles: a). Mesh grading; b). Solution profile

logarithmic scales in Figure 13 for the illustration of the decay of the relative errors as a function of the number of actual contact nodes.

\subsection{Relevance of the different error estimator contributions}

We consider three different experiments. In each of them the domain of computation is a unit cube of linear elastic material with the Young's modulus $E=5 \cdot 10^{2} \frac{\mathrm{kN}}{\mathrm{mm}^{2}}$ and the Poisson ratio $\nu=0.3$. One side of the cube is the Dirichlet boundary and the opposite side is the potential contact boundary so that the body can be pressed against the obstacle by means of non-zero Dirichlet values in direction of the obstacle. The remaining sides are Neumann boundary sides. The direction of constraints $\nu$ is perpendicular to the potential 


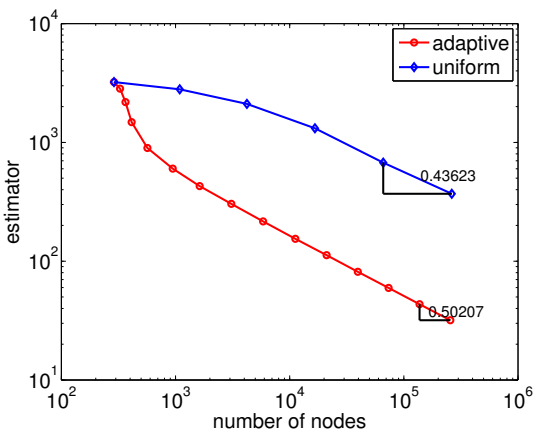

(a) Wedge example with quadrilaterals

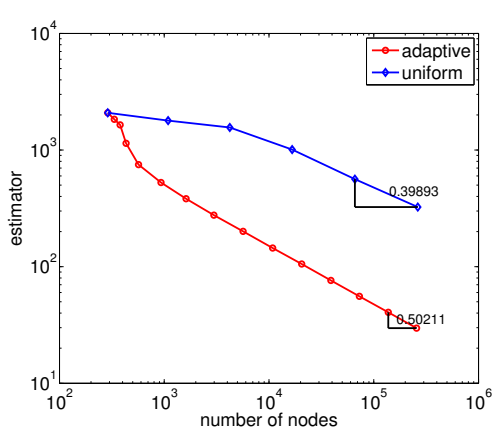

(b) Wedge example with triangles

Fig. 7: Estimator plotted against $\# N_{\mathfrak{m}_{k}}$ (logarithmic scales)

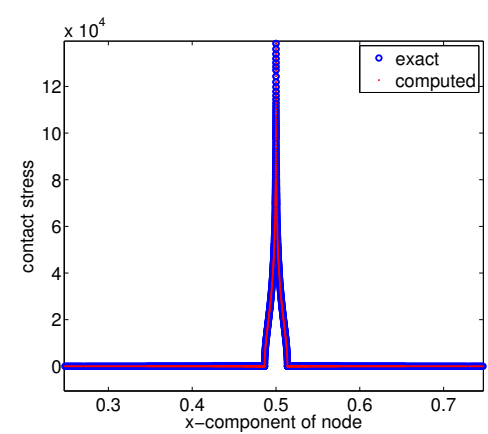

(a) Wedge example with quadrilaterals

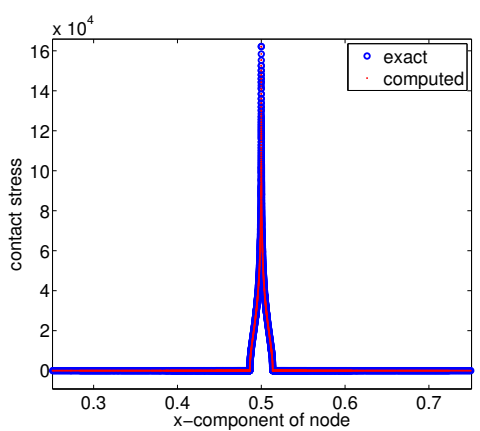

(b) Wedge example with triangles

Fig. 8: Approximation of contact stresses

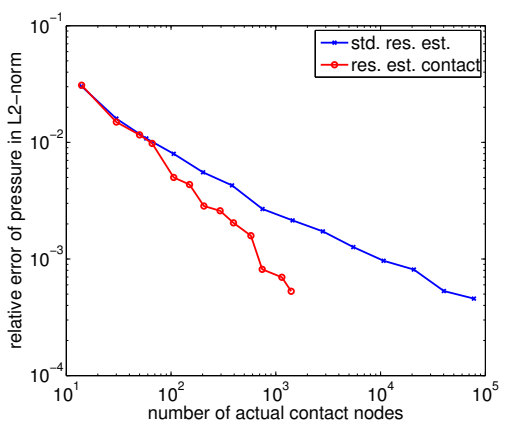

(a) Wedge example with quadrilaterals

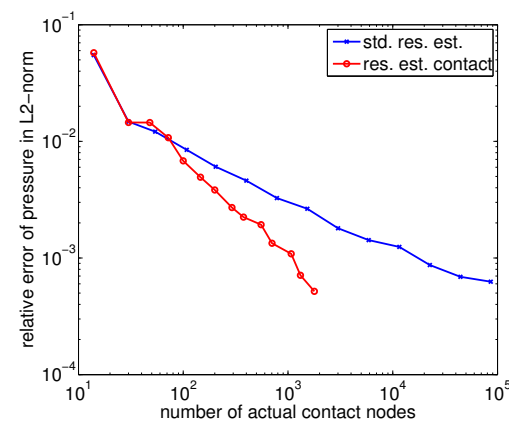

(b) Wedge example with triangles

Fig. 9: Relative error in $L^{2}\left(\Gamma_{C}\right)$ of discrete contact stresses using new residual-type estimator for contact as defined in (22) (red circles) and standard residual estimator (blue circles) plotted against $\# N_{\mathfrak{m}_{k}}^{\mathrm{aC}}$ 


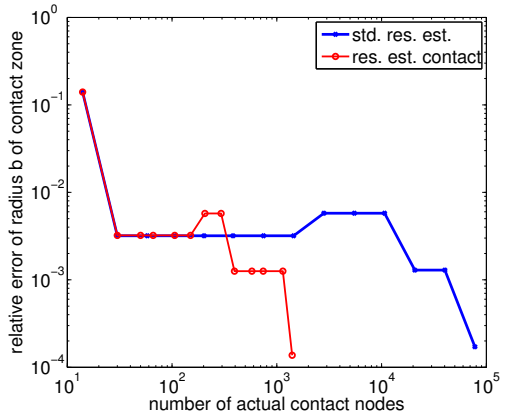

(a) Wedge example with quadrilaterals

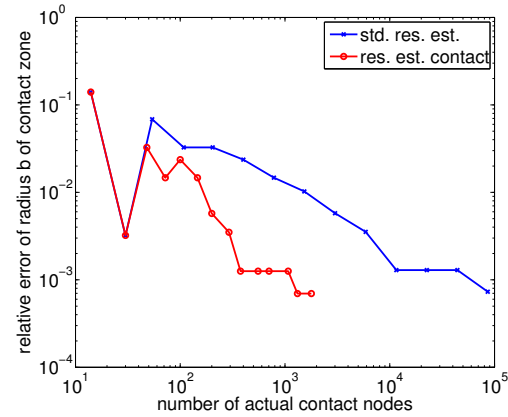

(b) Wedge example with triangles

Fig. 10: Relative error of radius of contact zone using new residual-type estimator for contact as defined in (22) (red circles) and standard residual estimator (blue circles) plotted against $\# N_{\mathfrak{m}_{k}}^{\mathrm{aC}}$

contact boundary. The Dirichlet values in direction $\nu$ are set to 0.05 or 0.08 depending on the obstacle. For the other directions we choose zero Dirichlet values.

In the first example of this section, called pyramid example, the obstacle is a pyramid so that the tip of the pyramid indents the elastic cube, see Figure 14(a). The starting grid consists primarily of pyramids. Figure 14(b) shows the solution on the adaptively refined grid around the actual contact boundary. The decay of the error estimator on the adaptively refined mesh is much faster as on the uniformly refined grid, see Figure 15, thus demonstrating clearly the merit of the error estimator. The experimental order of convergence of the error estimator is around 0.42 for the adaptively refined grid, see Figure 15 . The value is greater than $\frac{1}{d}$ which is known as optimal value. One possible explanation is that we are still in the pre-asymptotic regime where the approximation of the $(d-1)$-dimensional contact boundary is dominating.

The elements have been refined mostly along the cross where the edges of the pyramid indent the elastic body and around the free boundary zone, which is given by the curved lines surrounding the cross. The largest error estimator contributions for the adaptive refinement are $\eta_{1}$ (element residual), $\eta_{2}$ (inner jump residual) and $\eta_{5}$ (contact stress residual). The distribution of the local contributions $\eta_{1, p}^{2}$ and $\eta_{2, p}^{2}$ in refinement step 6 can be seen in Figure 16(a) and 16(b). From Figure 16 we can deduce that the refinement of the cross where the edges of the pyramid indent the elastic body is due to $\eta_{1}$ (element residual) and $\eta_{2}$ (inner jump residual). The contribution $\eta_{5}$ (contact stress residual) is mainly responsible for the adaptive refinement around the free boundary zone. The distribution of the local contributions $\eta_{5, p}^{2}$ in refinement step 6 is visualized in Figure 16(c).

Next, we consider an example where all additional error estimator contributions $\eta_{7}$ (obstacle approximation) and $\eta_{8}$ (actual constraint violation) are non-zero. The obstacle looks like a hat, see e.g., Figure 17(a). We call this experiment hat example and choose starting grids primarily consisting of pyramids. The final grid and the solution can be seen in a crosssection in Figure 17(b). The distribution of the local error estimator contributions $\eta_{5, p}^{2}, \eta_{6, p}^{2}$, $\eta_{7, p}^{2}$, and $\eta_{8, p}^{2}$ which are related to the contact constraints is visualized in Figure 18 in the fourth adaptive refinement step. The most important of these error estimator contributions is $\eta_{5}$ (contact stress residual) with a maximal local value of 0.03 for $\eta_{5, p}^{2}$ in refinement step 


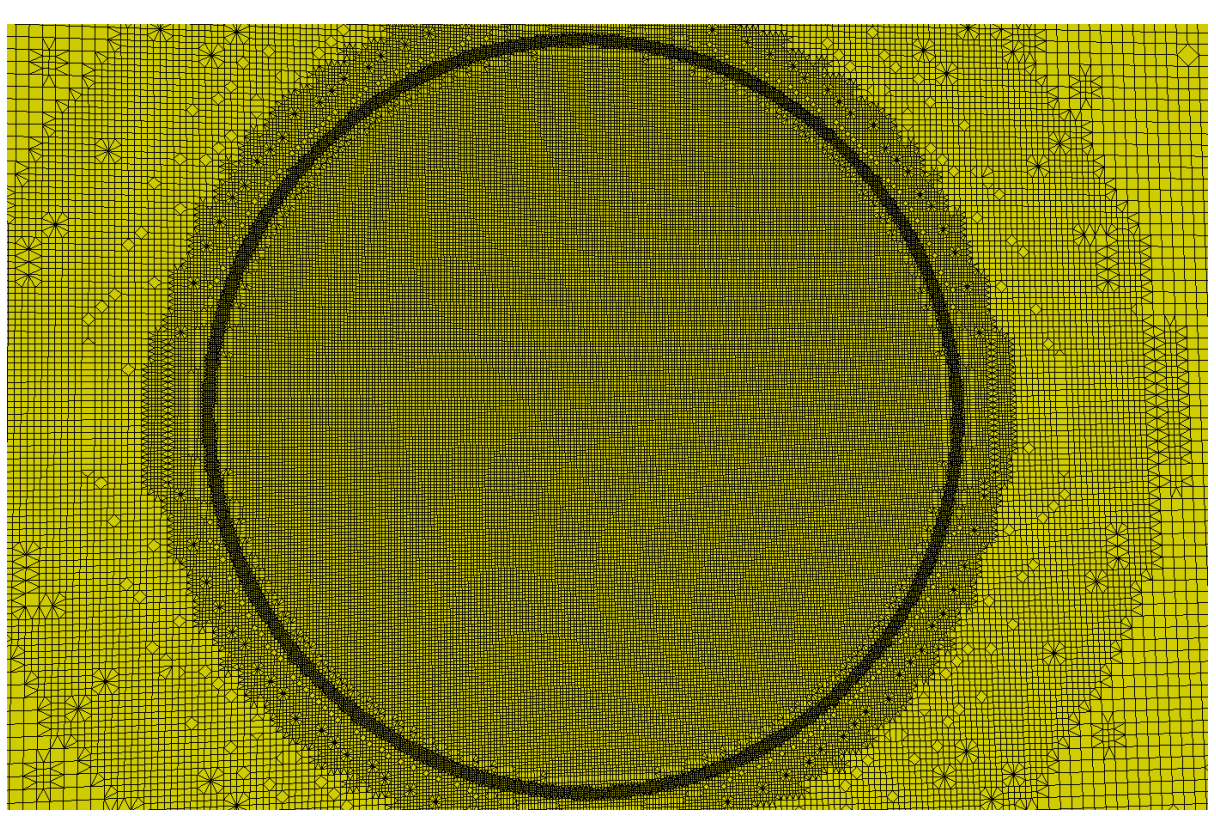

(a) Hertzian contact with hexahedra

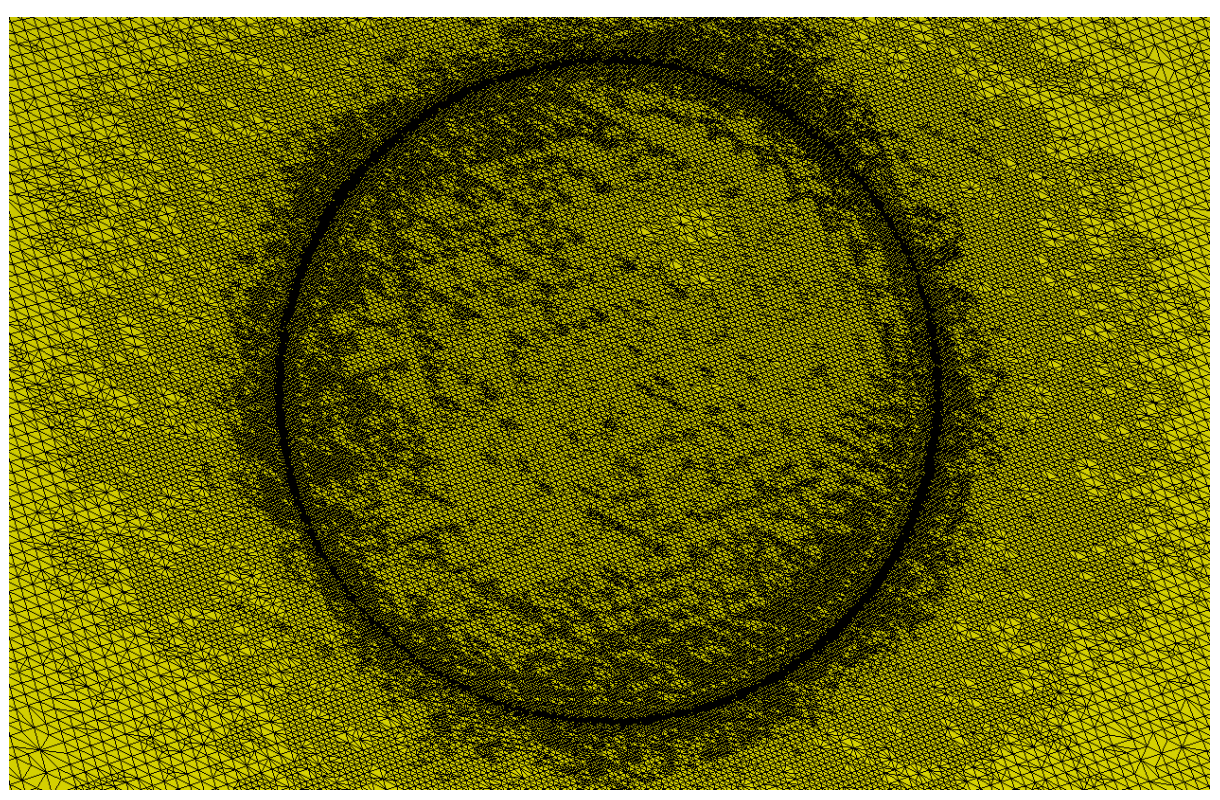

(b) Hertzian contact with tetrahedra

Fig. 11: Zoom in actual contact area of final grid on the contact boundary 


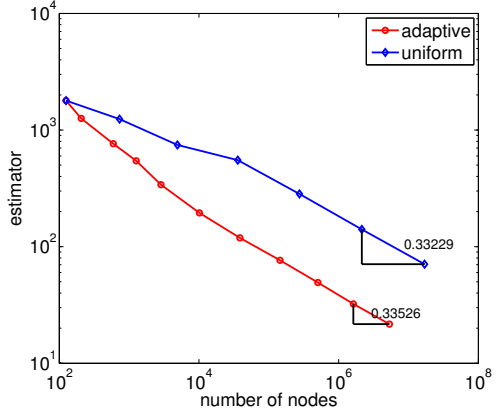

(a) Hertzian contact with hexahedra

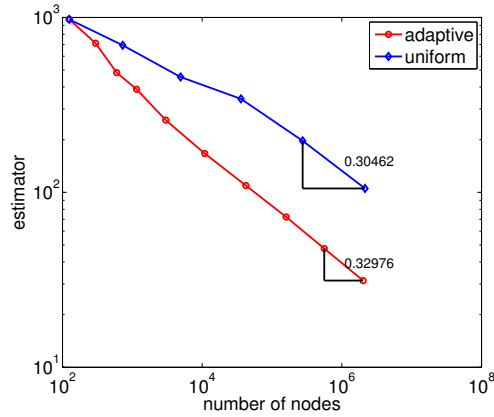

(b) Hertzian contact with tetrahedra

Fig. 12: Estimator plotted against $\# N_{\mathfrak{m}_{k}}$ (logarithmic scales)

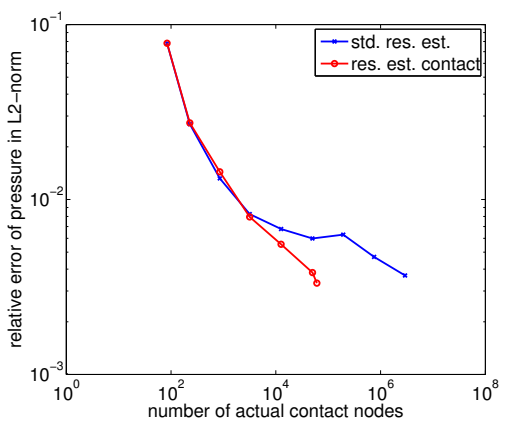

(a) Hertzian contact with hexahedra

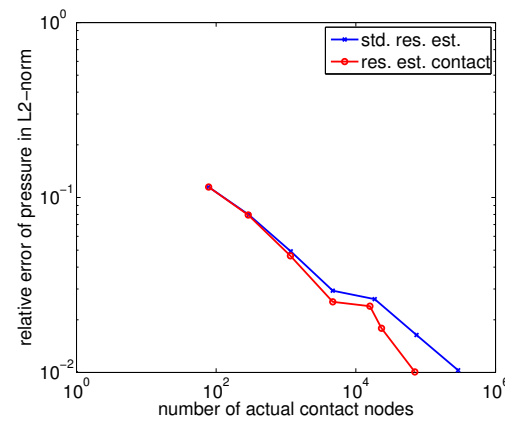

(b) Hertzian contact with tetrahedra

Fig. 13: Relative error in $L^{2}\left(\Gamma_{C}\right)$ of contact stresses using new residual-type estimator for contact as defined in (22) (red circles) and standard residual estimator (blue circles) plotted against $\# N_{\mathfrak{m}_{k}}^{\mathrm{aC}}$

4. From the definition of $\eta_{6}$ (complementarity residual) we know that the local contributions are restricted to the free boundary zone, see 18(c). Due to the shape of the hat and the structure of the grid the error estimator contributions $\eta_{7, p}, \eta_{8, p}$ are also located near the free boundary zone, compare Figure 18(d)-(e).

If we choose a sinus wave as obstacle (wave example) the importance of the additional error estimator contribution $\eta_{8}$ (actual constraint violation) gets evident. The starting grid consists of hexahedra. The grid projected from the contact boundary on the obstacle is shown for the starting grid and after three refinement steps in Figure 19. Due to the length of the sinus waves and due to the meshsize of the hexahedra on the starting grid, the grid around three of the four contact regions is well resolved but not of the fourth one on the left side. There the local error estimator contributions $\eta_{8, p}^{2}$ are non-zero, see Figure 19(c), and provoke further refinement and hence the detection of contact. 


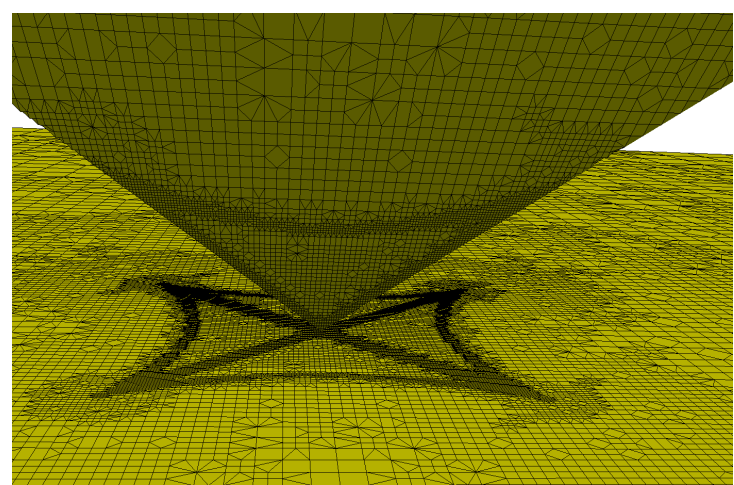

(a) Zoom of final grid of contact boundary, also projected on obstacle

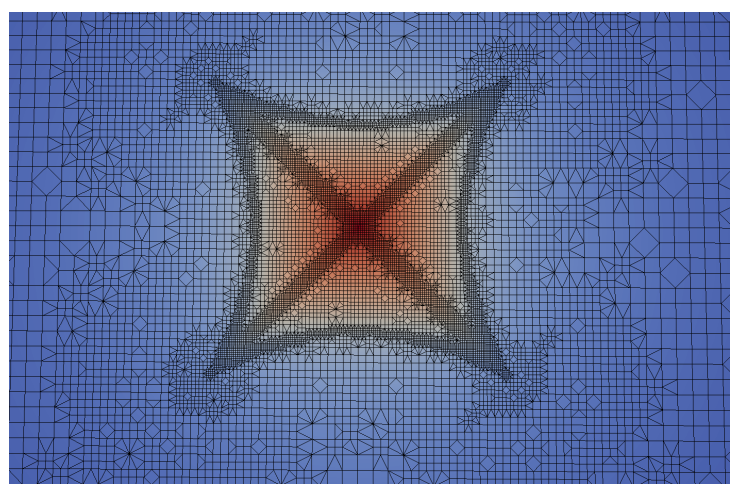

(b) Solution profile $\left(-u_{\nu}\right)$ on final grid around the actual contact boundary; ranges between -0.05 (blue) and 0.0 (red)

Fig. 14: Pyramid example

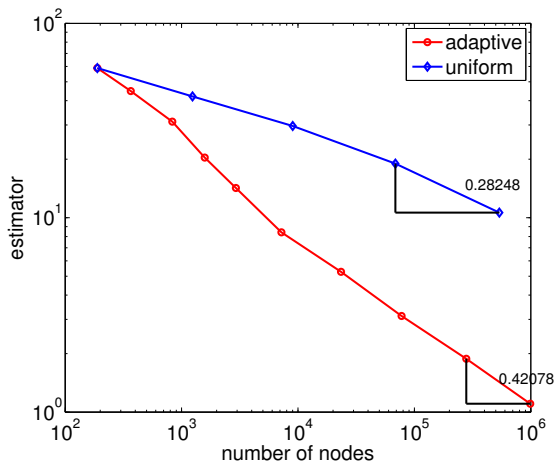

Fig. 15: Pyramid example: Estimator plotted against $\# N_{\mathfrak{m}_{k}}$ (logarithmic scales) 


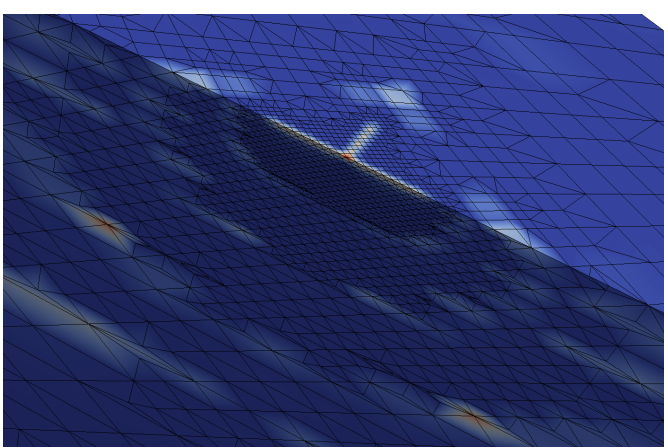

(a) $\eta_{1, p}^{2}$ (element residual); values are ranging between 0.0 (blue) and 0.07 (red)

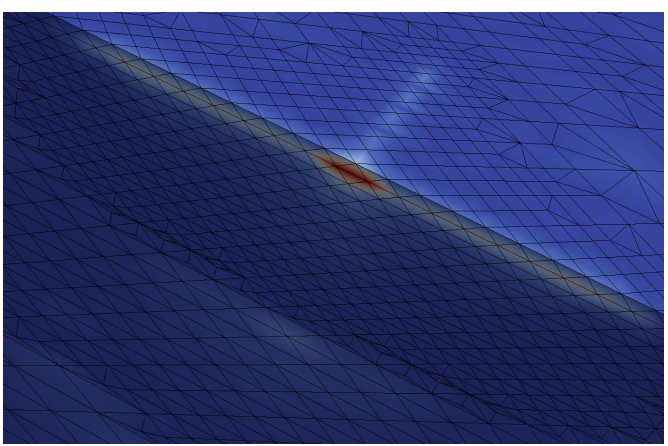

(b) $\eta_{2, p}^{2}$ (inner jump residual); values are ranging between $10^{-9}$ (blue) and 0.02 (red)

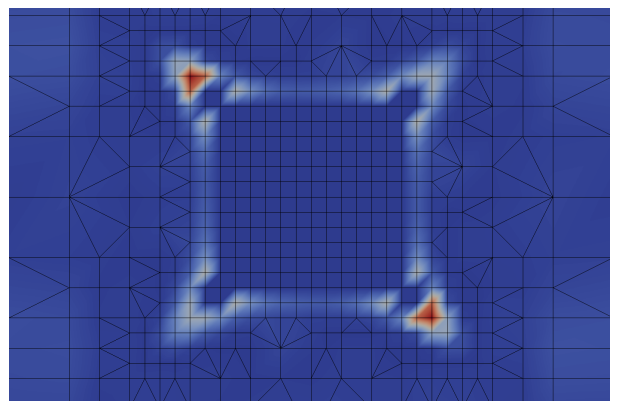

(c) $\eta_{5, p}^{2}$ (contact stress residual); values are ranging between $10^{-9}$ (blue) and 0.007 (red)

Fig. 16: Pyramid example: Local distribution of selected estimator contributions shown in a cut through the cube in a). and b). and on the contact boundary in c). after 6 steps of adaptive refinement 


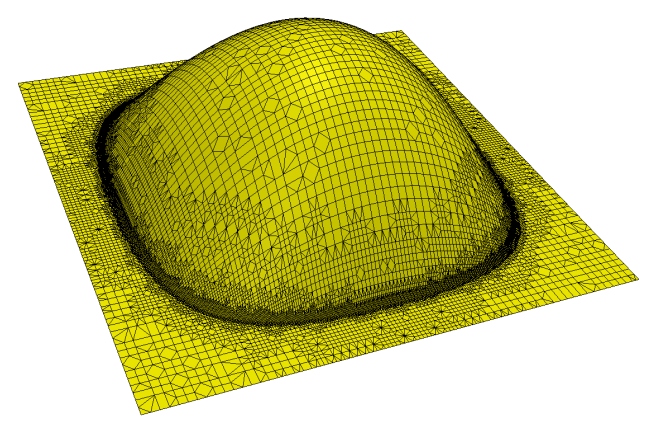

(a) Final grid of contact boundary projected on obstacle

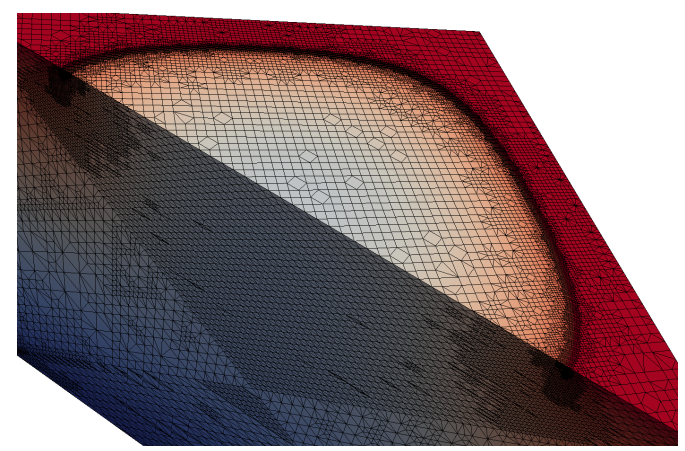

(b) Solution profile $(-\boldsymbol{u} \cdot \boldsymbol{\nu})$ over final grid shown in a cut through the cube

Fig. 17: Hat example

Acknowledgements One of the authors (M.W.) would like to thank the Bonn International Graduate School for financial support. Moreover this project was also supported by FORD, university research program, "Advanced numerical algorithms to improve high nonlinear crash simulation with multi-body contacts and friction" and by the BMBF-project ASIL (advanced solvers integrated library).

\section{References}

1. Ainsworth, M., Oden, J.: A posteriori error estimation in finite element analysis. Pure Appl. Math. Wiley, New York (2000)

2. Baiocchi, C.: Estimation d'erreur dans $L^{\infty}$ pour les inèquations à obstacle, in Mathematical Aspects of Finite Element Methods, Proc. Conf. Rome 1975, I. Galligani and E. Magenes, eds., vol. 606 of Lect. Notes Math., 27-34 (1977)

3. Bartels, S., Carstensen, C.: Averaging techniques yield reliable a posteriori finite element error control for obstacle problems. Numer. Math. 99, 225-249 (2004)

4. Bastian, P., Birken, K., Lang, S., Neuß, N., Rentz-Reichert, H., Wieners, C.: UG - A flexible software toolbox for solving partial differential equations. Comput. Vis. Sci. 1, 27-40 (1997)

5. Ben Belgacem, F., Bernardi, C., Blouza, A., Vohralík, M.: On the unilateral contact between membranes. Part 2: A posteriori analysis and numerical experiments. IMA J. Numer. Anal. 32, 1147-1172 (2012)

6. Braess, D.: A posteriori error estimators for obstacle problems-another look. Numer. Math. 101, 415$421(2005)$ 


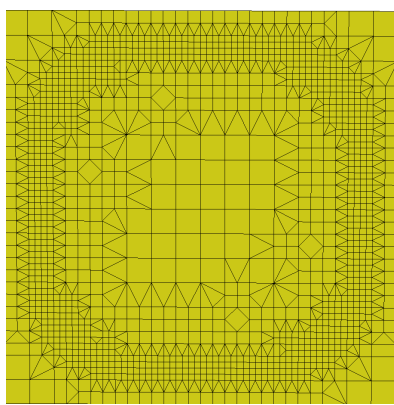

(a) Grid in refinement step 4

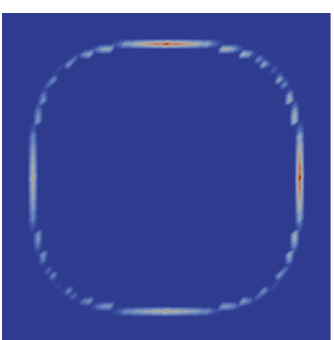

(c) $\eta_{6, p}^{2}$ in refinement step 4
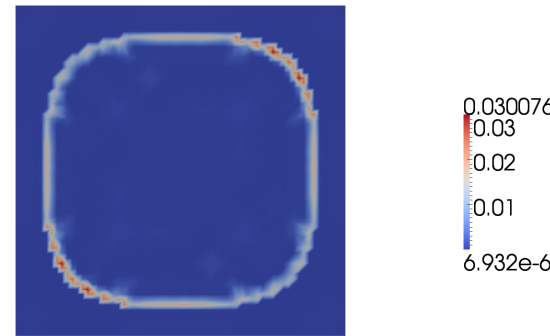

(b) $\eta_{5, p}^{2}$ in refinement step 4

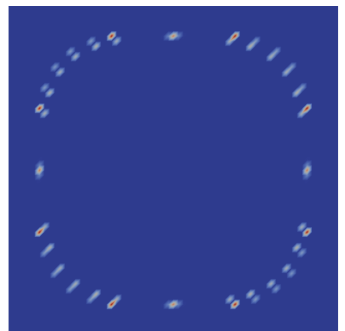

(d) $\eta_{7, p}^{2}$ in refinement step 4

$$
\begin{aligned}
& 8.62 e-13 \\
& 8 e-13 \\
& 6 e-13 \\
& 4 e-13 \\
& 2 e-13 \\
& 0
\end{aligned}
$$

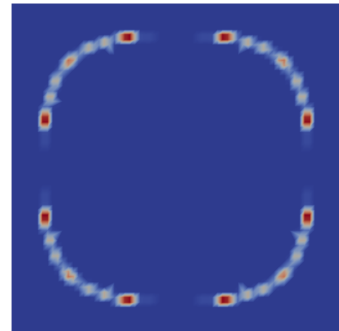

(e) $\eta_{8, p}^{2}$ in refinement step 4

Fig. 18: Hat example: Local distribution of selected estimator contributions on the contact boundary

7. Braess, D., Hoppe, R., Schöberl, J.: A posteriori estimators for obstacle problems by the hypercircle method. Comput. Vis. Sci. 11, 351-362 (2008)

8. Brezzi, F., Hager, W., Raviart, P.: Error estimates for the finite element solution of variational inequalities. Numer. Math. 28, 431-443 (1977)

9. Chen, Z., Nochetto, R.: Residual type a posteriori error estimates for elliptic obstacle problems. Numer Math. 84, 527-548 (2000)

10. Ciarlet, P.: Mathematical Elasticity. Vol. I, Stud. Math. Appl. 20 North-Holland Publishing Co., Amsterdam (1988)

11. Eck, C.: Existenz und Regularität der Lösungen für Kontaktprobleme mit Reibung. PhD thesis, Mathematisches Institut der Universität Stuttgart (1996)

12. Fierro, F., Veeser, A.: A posteriori error estimators for regularized total variation of characteristic functions. SIAM J. Numer. Anal. 41, 2032-2055 (2003)

13. Hertz, H.: Über die Berührung fester elastischer Körper. J. Reine Angew. Math. 92, 156-171 (1881)

14. Hild, P., Nicaise, S.: A posteriori error estimations of residual type for Signorini's problem. Numer. Math. 101, 523-549 (2005) 


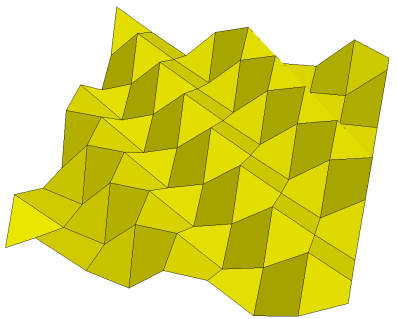

(a) Approximated obstacle over starting grid

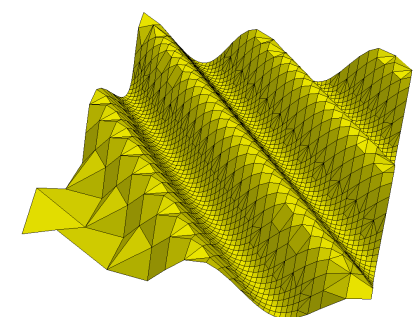

(b) Approximated obstacle after 3 steps of adaptive refinement

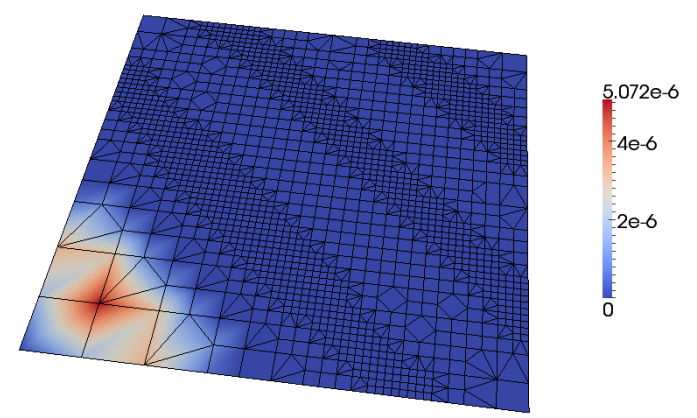

(c) $\eta_{8, p}^{2}$ (actual constraint violation) on the contact boundary in refinement step 3

Fig. 19: Wave example

15. Hild, P., Nicaise, S.: Residual a posteriori error estimators for contact problems in elasticity. M2AN Math. Model. Numer. Anal. 41, 897-923 (2007)

16. Hlaváček, I., Haslinger, J., Nečas, J., Lovíšek, J.: Solution of variational inequalities in mechanics, vol. 66 of Applied Mathematical Sciences. Springer-Verlag, New York (1988)

17. Hüeber, S., Wohlmuth, B.: An optimal a priori error estimate for nonlinear multibody contact problems. SIAM J. Numer. Anal. 43, 156-173 (2005)

18. Johnson, K.: Contact mechanics. Cambridge University Press (1985)

19. Kikuchi, N., Oden, J.: Contact problems in elasticity: a study of variational inequalities and finite element methods. SIAM, Philadelphia, PA (1988)

20. Kornhuber, R.: A posteriori error estimates for elliptic variational inequalities. Comput. Math. Appl. 31, 49-60 (1996)

21. Kornhuber, R.: Adaptive monotone multigrid methods for nonlinear variational problems. Adv. Numer. Math. Teubner, Stuttgart (1997)

22. Kornhuber, R., Zou, Q.: Efficient and reliable hierarchical error estimates for the discretization error of elliptic obstacle problems. Math. Comp. 80, 69-88 (2011)

23. Krause, R.: Monotone Multigrid Methods for Signorini's Problem with Friction. PhD thesis, Freie Universität Berlin (2000)

24. Moon, K., Nochetto, R., von Petersdorff, T., Zhang, C.: A posteriori error analysis for parabolic variational inequalities. M2AN Math. Model. Numer. Anal. 41, 485-511 (2007)

25. Nochetto, R., Siebert, K. and Veeser, A.: Pointwise a posteriori error control for elliptic obstacle problems. Numer. Math. 95, 163-195 (2003)

26. Nochetto, R., Siebert, K. and Veeser, A.: Fully localized a posteriori error estimators and barrier sets for contact problems. SIAM J. Numer. Anal. 42, 2118-2135 (2005) 
27. Repin, S.: Functional a posteriori estimates for elliptic variational inequalities. J. Math. Sci., 152, 702712 (2008)

28. Rodrigues, J.: Obstacle problems in mathematical physics. North-Holland Publishing Co., Amsterdam (1987)

29. Siebert, K., Veeser, A.: A unilaterally constrained quadratic minimization with adaptive finite elements SIAM J. Optim. 18, 260-289 (2007)

30. Steinbach, O.: Numerical approximation methods for elliptic boundary value problems. Springer, New York (2008)

31. Veeser, A.: Efficient and reliable a posteriori error estimators for elliptic obstacle problems. SIAM J. Numer. Anal. 39, 146-167 (2001)

32. Veeser, A., Verfürth, R.: Explicit upper bounds for dual norms of residuals. SIAM J. Numer. Anal. 47, 2387-2405 (2009)

33. Verfürth, R.: A Posteriori Error Estimation Techniques for Finite Element Methods. Oxford University Press (2013)

34. Verfürth, R.: A review of a posteriori error estimation techniques for elasticity problems. Comp. Meth. Appl. Mech. Engrg. 176, 419-440 (1999)

35. Walloth, M.: Adaptive numerical simulation of contact problems: Resolving local effects at the contact boundary in space and time. $\mathrm{PhD}$ thesis, Rheinische Friedrich-Wilhelms-Universität Bonn (2012)

36. Weiss, A., Wohlmuth, B.: A posteriori error estimator and error control for contact problems. Math. Comp. 78, 1237-1267 (2009)

37. Weiss, A., Wohlmuth, B.: A posteriori error estimator for obstacle problems. SIAM J. Sci. Comput. 32, 2627-2658 (2010)

38. Wohlmuth, B.: An a posteriori error estimator for two-body contact problems on non-matching meshes. J. Sci. Comput. 33, 25-45 (2007)

39. Zou, Q., Veeser, A., Kornhuber, R., Gräser, C.: Hierarchical error estimates for the energy functional in obstacle problems. Numer. Math. 117, 653-677 (2011) 